Review

\title{
An overview of methods for deriving the radiative transfer theory from the Maxwell equations. III: Effects of random rough boundaries and packing density
}

\author{
Adrian Doicu ${ }^{\mathrm{a}, *}$, Michael I. Mishchenko ${ }^{\mathrm{b}}$ \\ a Deutsches Zentrum für Luft- und Raumfahrt (DLR), Institut für Methodik der Fernerkundung (IMF), Oberpfaffenhofen, 82234, Germany \\ ${ }^{\mathrm{b}}$ NASA Goddard Institute for Space Studies, 2880, Broadway, New York, NY, 10025, USA
}

\section{A R T I C L E I N F O}

\section{Article history:}

Received 12 September 2018

Accepted 1 November 2018

Available online 6 November 2018

\section{Keywords:}

Radiative transfer theory

Discrete random media

Randomly rough interfaces

Wigner transform

On-shell approximation

\begin{abstract}
A B S T R A C T
In this paper we apply the Green function formalism and the Wigner transform method to derive the radiative transfer equation for a layer of sparse discrete random medium bounded by scattering rough interfaces. The radiative transfer equation is formulated with respect to the Wigner transform of the dyadic correlation function for the electromagnetic field. The results are obtained under the effective field and on-shell approximations, and the meaning of these approximations is discussed in detail.
\end{abstract}

(c) 2018 Elsevier Ltd. All rights reserved.

\section{Contents}

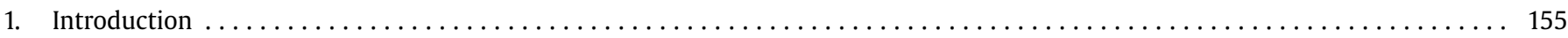

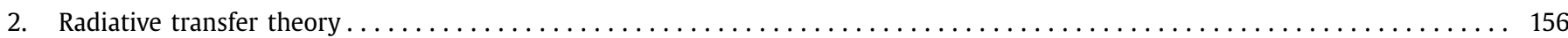

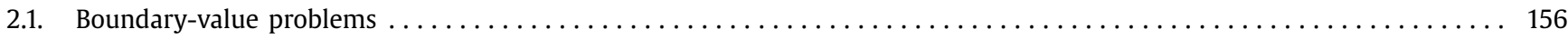

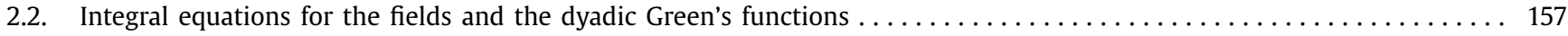

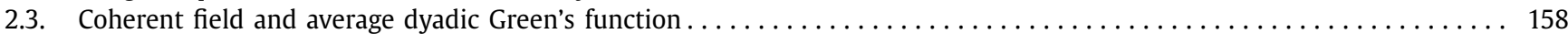

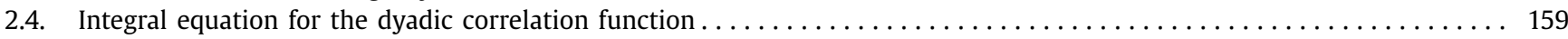

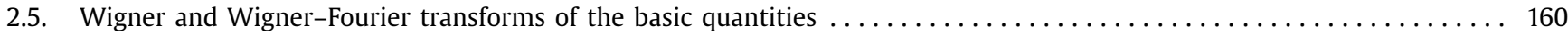

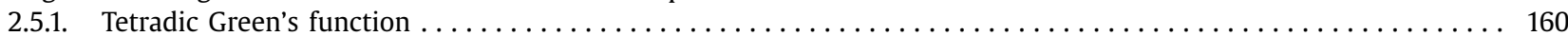

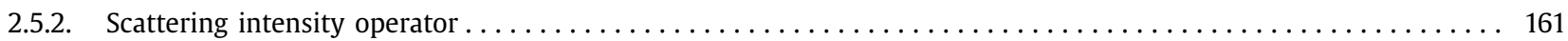

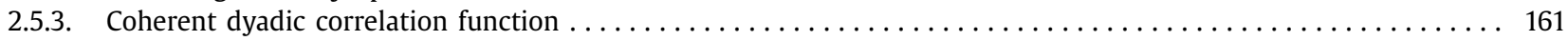

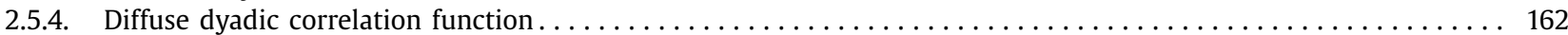

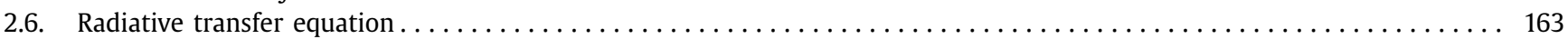

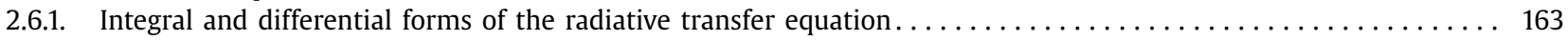

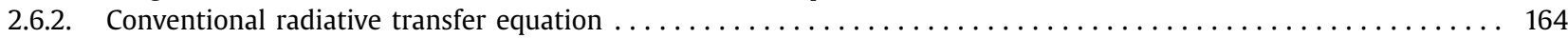

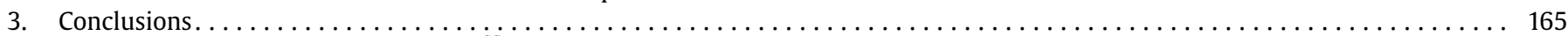

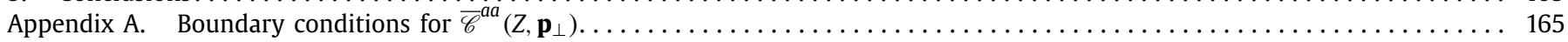

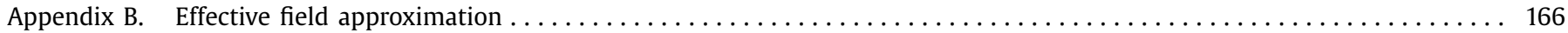

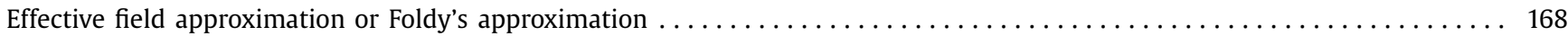

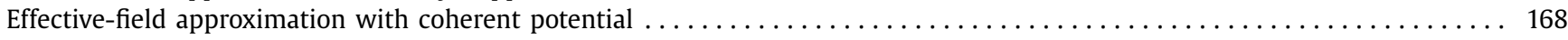

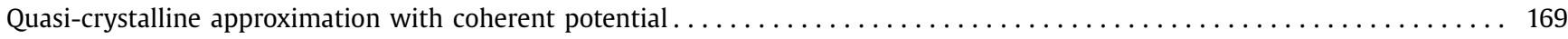

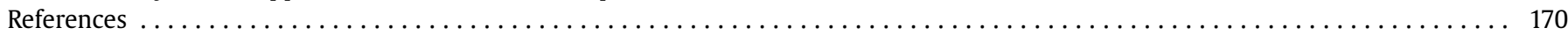

* Corresponding author.

E-mail address: adrian.doicu@dlr.de (A. Doicu). 


\section{Introduction}

The link between the electromagnetic scattering theory and the classical radiometry theory can be said to consist in a relationship between the dyadic correlation function $\overline{\mathbf{C}}$, which is a "spacespace" quantity, and the specific dyadic correlation function $\overline{\boldsymbol{\Sigma}}$, which is a "space-phase" quantity. In Refs. [1,2], we defined $\bar{\Sigma}$ through an angular spectrum representation for $\overline{\mathbf{C}}$. The procedure was to derive a series expansion for $\overline{\mathbf{C}}$ from which the angular spectrum representation was apparent; the resulting series expansion for $\bar{\Sigma}$ has led to a transport-type equation for this quantity. Fundamentally, the conceptual approach adopted in Ref. [1] follows that developed in Refs. $[3,4]$, wherein the randomness of the total electromagnetic field is a direct and explicit consequence of the randomness of particle positions. Hence, no ad hoc assumptions about the statistical properties of the field are required. Yet this approach can become cumbersome in the case of a discrete random medium with scattering rough boundaries.

An alternative, albeit less rigorous, way to establish the above link is to resort to ad hoc assumptions about statistical properties of the random electromagnetic field and consider the Wigner transform of the dyadic correlation function $\overline{\mathbf{C}}\left(\mathbf{r}, \mathbf{r}^{\prime}\right)=$ $\left\langle\mathbf{E}(\mathbf{r}) \otimes \mathbf{E}^{\star}\left(\mathbf{r}^{\prime}\right)\right\rangle_{V}[5-9]$, that is,

$\overline{\mathbf{C}}_{\mathrm{W}}(\mathbf{R}, \mathbf{p})=\int \overline{\mathbf{C}}\left(\mathbf{R}+\frac{\rho}{2}, \mathbf{R}-\frac{\rho}{2}\right) \mathrm{e}^{-\mathrm{j} \mathbf{p} \cdot \rho} \mathrm{d}^{3} \boldsymbol{\rho}$,

where $\mathbf{R}=\left(\mathbf{r}+\mathbf{r}^{\prime}\right) / 2$ and $\rho=\mathbf{r}-\mathbf{r}^{\prime}$ are the "center of gravity" and the difference variable, respectively, $\otimes$ is the dyadic product sign, $\star$ stands for complex conjugate, and $\langle\cdot\rangle_{V}$ denotes the average over volumetric fluctuations of the field at points $\mathbf{r}$ and $\mathbf{r}^{\prime}$ implicitly caused by randomly varying particle positions. In Refs. [7,8], the Wigner function (1) is called the local spectrum of the random field, and it is noted that in the presence of statistical inhomogeneities, the Wigner function can take on negative values. Therefore, in the general case, an energy interpretation cannot be attributed to this quantity. Only upon making an a priori assumption that the random field is quasi-uniform, the Wigner function becomes a measure of the intensity fluctuations. In the quasi-uniform field approximation it is assumed that the dyadic correlation function varies weakly with $\mathbf{R}$ and varies strongly with $\rho$. When this assumption is made and the evanescent waves are neglected, the Wigner transform is of the form

$\overline{\mathbf{C}}_{\mathrm{W}}(\mathbf{R}, \mathbf{p}) \propto \delta\left(p-K^{\prime}\right) \overline{\mathbf{\Sigma}}(\mathbf{R}, \widehat{\mathbf{p}})$,

so that by applying the inverse Wigner transform, we obtain the angular spectrum representation

$\overline{\mathbf{C}}\left(\mathbf{R}+\frac{\rho}{2}, \mathbf{R}-\frac{\rho}{2}\right) \propto \int \overline{\boldsymbol{\Sigma}}(\mathbf{R}, \widehat{\mathbf{p}}) \mathrm{e}^{\mathrm{j} \mathrm{K}^{\prime} \widehat{\mathbf{p}} \cdot \rho} \mathrm{d}^{2} \widehat{\mathbf{p}}$,

where $K$ is the effective wavenumber, $K^{\prime}=\operatorname{Re}(K), K^{\prime \prime}=\operatorname{Im}(K)$, and $\delta$ is the delta function. (Note that upright bold characters with carets hereinafter denote unit vectors.) Thus, by way of Eq. (2), the Wigner transform $\overline{\mathbf{C}}_{W}$ reproduces the specific dyadic correlation function $\bar{\Sigma}$ in the case $p=K^{\prime}$; therefore, an alternative procedure is to derive a transport-type equation for $\overline{\mathbf{C}}_{\mathrm{w}}$.

In describing the radiative transfer in discrete random media, we distinguish two distance scales. The long-distance scale is characterized by the mean free path $l_{\text {mp }}=1 /\left(2 K^{\prime \prime}\right)$, while the shortdistance scale is characterized by the effective wavelength scale $\lambda_{\mathrm{e}}=2 \pi / K^{\prime}[10,11]$. Transport type equations are derived based on the assumption that $l_{\mathrm{mp}} \gg \lambda_{\mathrm{e}}$, that is, the mean free path length is much larger than the wavelength scale. The quasi-uniform field approximation implies that the scale lengths of the dyadic correlation function with respect to $\mathbf{R}$ and $\rho$ are the mean free path $l_{\mathrm{mp}}$ and the wavelength scale $\lambda_{e}$, respectively.
In the low-frequency limit, the radiative transfer equation for a half-space of random, densely-distributed particles has been derived in Refs. $[10,11]$, and for a layer with plane boundaries in Ref. [12]. The effective wavenumber is computed from the Dyson equation by using the quasi-crystalline approximation with coherent potential, while the radiative transfer equation is gained from the Bethe-Salpeter equation for the diffuse dyadic correlation function $\left\langle\mathscr{E}(\mathbf{r}) \otimes \mathscr{E}^{\star}\left(\mathbf{r}^{\prime}\right)\right\rangle_{Y}$ under the ladder approximation, where $\mathscr{E}(\mathbf{r})$ is the diffuse (incoherent) scattered field. The solution method is based on a plane wave representation of the diffuse field, which in turn, yields an integral representation for $\left\langle\mathscr{E}(\mathbf{r}) \otimes \mathscr{E}^{\star}\left(\mathbf{r}^{\prime}\right)\right\rangle_{Y}$ in terms of the dyadic correlation function for the amplitudes of the plane waves. The radiative transfer equation is formulated with respect to the dyadic correlation function for the plane wave amplitudes, and is obtained by balancing the terms with the same phase dependence on the left- and the right-hand side of the BetheSalpeter equation. Actually, in the integral term of the BetheSalpeter equation, only the constructive interference terms varying on the long-distance scale $l_{\text {mp }}$ are retained; the destructive interference terms varying on the short-distance scale $\lambda_{e}$ are disregard. Physically, constructive interference terms correspond to waves traveling over the same paths, while destructive interference terms correspond to waves traveling along mutually opposite paths. This solution method does not use the Wigner transform; rather it is based on the application of the two-dimensional Fourier transform to the Bethe-Salpeter equation along the transverse component $\boldsymbol{\rho}_{\perp}$ of the difference variable $\boldsymbol{\rho}$.

A general approach for analyzing the radiative transfer in a densely-packed discrete random layer with rough boundaries has been developed by Soubret and Berginc $[13,14]$. That approach uses scattering operators to describe the wave interaction with the boundaries [15], and is based on a Green function formalism. In particular, two kinds of Green functions are introduced: the Green function $\overline{\mathbf{G}}\left(\mathbf{r}, \mathbf{r}^{\prime}\right)$ describing the field scattered by the layer containing particles and by the rough surfaces, and the Green function $\overline{\mathbf{G}}_{\mathrm{K}}\left(\mathbf{r}, \mathbf{r}^{\prime}\right)$ describing the field scattered by a homogeneous layer with rough boundaries and being characterized by an effective wavenumber $K$. The latter is expressed as a function of the rough surface scattering operators. The derivation is based on the correlated ladder-approximated Bethe-Salpeter equation for the tetradic $\left\langle\overline{\mathbf{G}}\left(\mathbf{r}, \mathbf{r}^{\prime}\right) \otimes \overline{\mathbf{G}}^{\star}\left(\mathbf{r}, \mathbf{r}^{\prime}\right)\right\rangle_{V S}$, where $\langle\cdot\rangle_{S}$ means the average over surface fluctuations and $\langle\langle\cdot\rangle\rangle_{V S}$ means the average over volumetric and surface fluctuations. The effective wavenumber and the intensity operator are computed in the framework of the quasi-crystalline approximation with coherent potential. By applying the Wigner transform to the Bethe-Salpeter equation and differentiating the resulting equation, a radiative transfer equation for the Wigner transform of $\left\langle\overline{\mathbf{G}}\left(\mathbf{r}, \mathbf{r}^{\prime}\right) \otimes \overline{\mathbf{G}}^{\star}\left(\mathbf{r}, \mathbf{r}^{\prime}\right)\right\rangle_{V S}$ is obtained. This is shown to be equivalent to a radiative transfer equation for the tetradic diffuse specific intensity. The source term of this equation depends on the tetradic reduced intensity which is defined as the dyadic product of the scattering operators at the rough interface (the interface between the layer and the medium containing the sources of the incident field).

A similar approach based on surface scattering operators and Wigner transform was used by Mudaliar [16]. However, in this case, (i) the starting point is the Bethe-Salpeter equation for the dyadic correlation function $\left\langle\mathbf{E}(\mathbf{r}) \otimes \mathbf{E}^{\star}\left(\mathbf{r}^{\prime}\right)\right\rangle_{V S}$, (ii) the effective wavenumber and the intensity operator are computed in the framework of the effective field approximation, and (iii) the radiative transfer equation is formulated for the Wigner transform of $\left\langle\mathbf{E}(\mathbf{r}) \otimes \mathbf{E}^{\star}\left(\mathbf{r}^{\prime}\right)\right\rangle_{V S^{\prime}}$. Thus, the analysis corresponds to a discrete random layer with a sparse concentration of particles.

All methods described above are based on a priori assumptions about the random electromagnetic field, including the effec- 


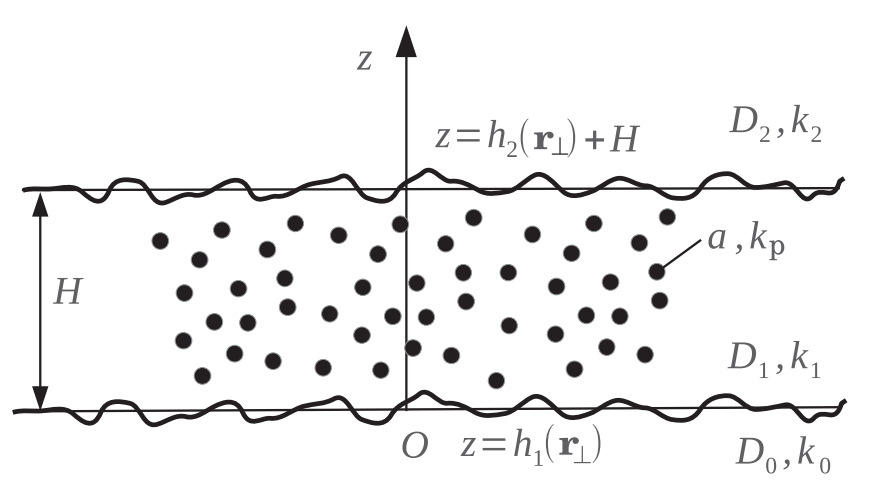

Fig. 1. Discrete random layer with rough boundaries.

tive field approximation. According to this approximation, the coherent field in a discrete random medium behaves as a wave in a homogeneous medium with an effective propagation constant. In this paper, we revisit the approach used by Mudaliar [16] for analyzing radiative transfer in a layer of discrete random medium with rough boundaries and a sparse concentration of particles. Our goal is to clarify the underlying assumptions and to discuss the meaning of the effective field approximation.

\section{Radiative transfer theory}

We consider a discrete random medium with boundaries described by the random functions $z=h_{1}\left(\mathbf{r}_{\perp}\right)$ and $z=H+h_{2}\left(\mathbf{r}_{\perp}\right)$ as shown in Fig. 1 . The domains $D_{0}$ and $D_{2}$ are homogeneous media with permittivities $\varepsilon_{0}$ and $\varepsilon_{2}$, respectively. The random medium is made of $N$ identical spherical particles of radius $a$ and permittivity $\varepsilon_{\mathrm{p}}$ placed in the background medium $D_{1}$ of permittivity $\varepsilon_{1}$. The particles are centered at $\mathbf{R}_{1}, \mathbf{R}_{2}, \ldots, \mathbf{R}_{N}$, and the system of particles is characterized by the number concentration (the number of particles per unit volume) $n_{0}=N / V$, where $V$ is the volume accessible to the particles. All domains are nonmagnetic and characterized by the permeability $\mu_{0}$. The wave numbers in the domains $D_{0}$ and $D_{2}$ are $k_{0}^{2}=\omega^{2} \mu_{0} \varepsilon_{0}$ and $k_{2}^{2}=\omega^{2} \mu_{0} \varepsilon_{2}$, respectively, while the wave number in the random medium $D_{1}$ is

$k_{V}^{2}(\mathbf{r})=k_{1}^{2}+\sum_{i} k_{1}^{2}\left(\mathrm{~m}^{2}-1\right) \Theta\left(\mathbf{r}-\mathbf{R}_{i}\right)$,

where $i$ numbers the $N$ particles, $k_{1}^{2}=\omega^{2} \mu_{0} \varepsilon_{1}, \omega$ is the angular frequency, $\mathrm{m}=k_{\mathrm{p}} / k_{1}$ is the relative refractive index of the particles with respect to the background medium,

$\Theta(\mathbf{r})=\left\{\begin{array}{ll}1, & \mathbf{r} \in D_{\mathrm{p}} \\ 0, & \mathbf{r} \notin D_{\mathrm{p}}\end{array}\right.$,

and $D_{\mathrm{p}}$ is the domain occupied by a particle centered at the origin of the coordinate system (a sphere of radius $a$ ). The incident wave is a plane electromagnetic wave of amplitude $\mathcal{E}_{0}(\widehat{\mathbf{s}})$ propagating in the medium $D_{0}$ in the up-going direction $\widehat{\mathbf{s}}=\widehat{\mathbf{s}}_{\perp}+\widehat{S}_{\mathrm{z}} \widehat{\mathbf{z}}=\widehat{s_{\mathrm{x}}} \widehat{\mathbf{x}}+\widehat{s_{\mathrm{y}}} \widehat{\mathbf{y}}+$ $\widehat{S_{z}} \widehat{\mathbf{z}}$, that is,

$\mathbf{E}_{0}(\mathbf{r})=\left[\mathcal{E}_{0 \theta} \widehat{\boldsymbol{\theta}}(\widehat{\mathbf{s}})+\mathcal{E}_{0 \varphi} \widehat{\boldsymbol{\varphi}}(\widehat{\mathbf{s}})\right] \mathrm{e}^{\mathrm{j} \mathbf{k}_{0 \mathrm{~s}} \cdot \mathbf{r}}$

In Eq. (5), $\widehat{\boldsymbol{\theta}}(\widehat{\mathbf{s}})$ and $\widehat{\boldsymbol{\varphi}}(\widehat{\mathbf{s}})$ are the unit polar-coordinate vectors of the direction $\widehat{\mathbf{s}}$, and $\mathbf{k}_{0 \mathrm{~s}}$ is the wave vector; it is given by $\mathbf{k}_{0 \mathrm{~s}}=k_{0} \widehat{\mathbf{s}}=\mathbf{k}_{0 \mathrm{~s} \perp}+k_{0 \mathrm{z}}\left(\mathbf{k}_{0 \mathrm{~s} \perp}\right) \widehat{\mathbf{z}}$, with $\mathbf{k}_{0 \mathrm{~s} \perp}=k_{0} \widehat{\mathbf{s}}_{\perp}=k_{0}\left(\widehat{s_{\mathrm{x}}} \widehat{\mathbf{x}}+\widehat{s}_{\mathrm{y}} \widehat{\mathbf{y}}\right)$ and $k_{0 \mathrm{z}}\left(\mathbf{k}_{0 \mathrm{~s} \perp}\right)=k_{0} \widehat{s}_{\mathrm{z}}$.

In the following, we assume that the properties of the rough surfaces and the discrete random medium are statistically independent, which means that $\langle\langle\cdot\rangle\rangle_{V S}=\left\langle\langle\cdot\rangle_{V}\right\rangle_{S}=\left\langle\langle\cdot\rangle_{S}\right\rangle_{V}$.

\subsection{Boundary-value problems}

The following are the equations that govern the fields in each medium:

$$
\begin{aligned}
& \nabla \times \nabla \times \mathbf{E}^{0}(\mathbf{r})-k_{0}^{2} \mathbf{E}^{0}(\mathbf{r})=0, \\
& \nabla \times \nabla \times \mathbf{E}^{1}(\mathbf{r})-k_{V}^{2} \mathbf{E}^{1}(\mathbf{r})=0, \\
& \nabla \times \nabla \times \mathbf{E}^{2}(\mathbf{r})-k_{2}^{2} \mathbf{E}^{2}(\mathbf{r})=0,
\end{aligned}
$$

where $\mathbf{E}^{i}$ is the field in domain $D_{i}, i=1,2,3$. The boundary conditions on the lower and upper rough surfaces are

$\widehat{\mathbf{n}}_{1}(\mathbf{r}) \times \mathbf{E}^{0}(\mathbf{r})=\widehat{\mathbf{n}}_{1}(\mathbf{r}) \times \mathbf{E}^{1}(\mathbf{r})$,

$\widehat{\mathbf{n}}_{1}(\mathbf{r}) \times\left[\nabla \times \mathbf{E}^{0}(\mathbf{r})\right]=\widehat{\mathbf{n}}_{1}(\mathbf{r}) \times\left[\nabla \times \mathbf{E}^{1}(\mathbf{r})\right]$,

and

$\widehat{\mathbf{n}}_{2}(\mathbf{r}) \times \mathbf{E}^{1}(\mathbf{r})=\widehat{\mathbf{n}}_{2}(\mathbf{r}) \times \mathbf{E}^{2}(\mathbf{r})$,

$\widehat{\mathbf{n}}_{2}(\mathbf{r}) \times\left[\nabla \times \mathbf{E}^{1}(\mathbf{r})\right]=\widehat{\mathbf{n}}_{2}(\mathbf{r}) \times\left[\nabla \times \mathbf{E}^{2}(\mathbf{r})\right]$,

respectively. These systems are supplemented by the radiation condition well away from the layer. In Eqs. (9)-(10), $\mathbf{r}=\mathbf{r}_{\perp}+h_{1}\left(\mathbf{r}_{\perp}\right) \widehat{\mathbf{z}}$ and $\widehat{\mathbf{n}}_{1}(\mathbf{r})$ is the local unit vector normal to the rough surface $z=$ $h_{1}\left(\mathbf{r}_{\perp}\right)$ pointing toward $D_{0}$, while in Eqs. (11)-(12), $\mathbf{r}=\mathbf{r}_{\perp}+[H+$ $\left.h_{2}\left(\mathbf{r}_{\perp}\right)\right] \widehat{\mathbf{z}}$ and $\widehat{\mathbf{n}}_{2}(\mathbf{r})$ is the local unit vector normal to the rough surface $z=h_{2}\left(\mathbf{r}_{\perp}\right)$ pointing toward $D_{2}$. For a source point $\mathbf{r}^{\prime}$ in $D_{1}$, the dyadic Green's functions $\overline{\mathbf{G}}^{01}\left(\mathbf{r}, \mathbf{r}^{\prime}\right), \overline{\mathbf{G}}^{11}\left(\mathbf{r}, \mathbf{r}^{\prime}\right)$, and $\overline{\mathbf{G}}^{21}\left(\mathbf{r}, \mathbf{r}^{\prime}\right)$ describe the fields scattered by the rough surfaces and the discrete random medium at the field point $\mathbf{r}$ in the domains $D_{0}, D_{1}$, and $D_{2}$, respectively. They satisfy a boundary-value problem consisting in the dyadic wave equations

$\nabla \times \nabla \times \overline{\mathbf{G}}^{01}\left(\mathbf{r}, \mathbf{r}^{\prime}\right)-k_{0}^{2} \overline{\mathbf{G}}^{01}\left(\mathbf{r}, \mathbf{r}^{\prime}\right)=0$,

$\nabla \times \nabla \times \overline{\mathbf{G}}^{11}\left(\mathbf{r}, \mathbf{r}^{\prime}\right)-k_{V}^{2} \overline{\mathbf{G}}^{11}\left(\mathbf{r}, \mathbf{r}^{\prime}\right)=\delta\left(\mathbf{r}-\mathbf{r}^{\prime}\right) \overline{\mathbf{I}}$

$\nabla \times \nabla \times \overline{\mathbf{G}}^{21}\left(\mathbf{r}, \mathbf{r}^{\prime}\right)-k_{2}^{2} \overline{\mathbf{G}}^{21}\left(\mathbf{r}, \mathbf{r}^{\prime}\right)=0$,

where $\delta\left(\mathbf{r}-\mathbf{r}^{\prime}\right)$ is the three-dimensional delta function and $\overline{\mathbf{I}}$ is the identity dyadic, and the boundary conditions

$\widehat{\mathbf{n}}_{1}(\mathbf{r}) \times \overline{\mathbf{G}}^{01}\left(\mathbf{r}, \mathbf{r}^{\prime}\right)=\widehat{\mathbf{n}}_{1}(\mathbf{r}) \times \overline{\mathbf{G}}^{11}\left(\mathbf{r}, \mathbf{r}^{\prime}\right)$,

$\widehat{\mathbf{n}}_{1}(\mathbf{r}) \times\left[\nabla \times \overline{\mathbf{G}}^{01}\left(\mathbf{r}, \mathbf{r}^{\prime}\right)\right]=\widehat{\mathbf{n}}_{1}(\mathbf{r}) \times\left[\nabla \times \overline{\mathbf{G}}^{11}\left(\mathbf{r}, \mathbf{r}^{\prime}\right)\right]$,

and

$\widehat{\mathbf{n}}_{2}(\mathbf{r}) \times \overline{\mathbf{G}}^{11}\left(\mathbf{r}, \mathbf{r}^{\prime}\right)=\widehat{\mathbf{n}}_{2}(\mathbf{r}) \times \overline{\mathbf{G}}^{21}\left(\mathbf{r}, \mathbf{r}^{\prime}\right)$,

$\widehat{\mathbf{n}}_{2}(\mathbf{r}) \times\left[\nabla \times \overline{\mathbf{G}}^{11}\left(\mathbf{r}, \mathbf{r}^{\prime}\right)\right]=\widehat{\mathbf{n}}_{2}(\mathbf{r}) \times\left[\nabla \times \overline{\mathbf{G}}^{21}\left(\mathbf{r}, \mathbf{r}^{\prime}\right)\right]$,

on the lower and upper rough surfaces, respectively.

We assume that the effective wavenumber $K$ in the domain $D_{1}$ is known, and denote by $\mathbf{E}_{\mathrm{K}}^{0}(\mathbf{r}), \mathbf{E}_{\mathrm{K}}^{1}(\mathbf{r})$, and $\mathbf{E}_{\mathrm{K}}^{2}(\mathbf{r})$ the fields describing the scattering by a homogeneous layer with wavenumber $K$ and the same rough boundaries. These fields satisfy the wave equations (6)-(8) with $k_{V}$ replaced by $K$, i.e.

$\nabla \times \nabla \times \mathbf{E}_{\mathrm{K}}^{0}(\mathbf{r})-k_{0}^{2} \mathbf{E}_{\mathrm{K}}^{0}(\mathbf{r})=0$, 
$\nabla \times \nabla \times \mathbf{E}_{\mathrm{K}}^{1}(\mathbf{r})-K^{2} \mathbf{E}_{\mathrm{K}}^{1}(\mathbf{r})=0$,

$\nabla \times \nabla \times \mathbf{E}_{\mathrm{K}}^{2}(\mathbf{r})-k_{2}^{2} \mathbf{E}_{\mathrm{K}}^{2}(\mathbf{r})=0$,

and the boundary conditions (9)-(12). The pertinent dyadic Green's functions are $\overline{\mathbf{G}}_{\mathrm{K}}^{01}\left(\mathbf{r}, \mathbf{r}^{\prime}\right), \overline{\mathbf{G}}_{\mathrm{K}}^{11}\left(\mathbf{r}, \mathbf{r}^{\prime}\right)$, and $\overline{\mathbf{G}}_{\mathrm{K}}^{21}\left(\mathbf{r}, \mathbf{r}^{\prime}\right)$ solving the dyadic wave equations

$\nabla \times \nabla \times \overline{\mathbf{G}}_{\mathrm{K}}^{01}\left(\mathbf{r}, \mathbf{r}^{\prime}\right)-k_{0}^{2} \overline{\mathbf{G}}_{\mathrm{K}}^{01}\left(\mathbf{r}, \mathbf{r}^{\prime}\right)=0$,

$\nabla \times \nabla \times \overline{\mathbf{G}}_{\mathrm{K}}^{11}\left(\mathbf{r}, \mathbf{r}^{\prime}\right)-K^{2} \overline{\mathbf{G}}_{\mathrm{K}}^{11}\left(\mathbf{r}, \mathbf{r}^{\prime}\right)=\delta\left(\mathbf{r}-\mathbf{r}^{\prime}\right) \overline{\mathbf{I}}$,

$\nabla \times \nabla \times \overline{\mathbf{G}}_{\mathrm{K}}^{21}\left(\mathbf{r}, \mathbf{r}^{\prime}\right)-k_{2}^{2} \overline{\mathbf{G}}_{\mathrm{K}}^{21}\left(\mathbf{r}, \mathbf{r}^{\prime}\right)=0$,

and the boundary conditions (16)-(19).

2.2. Integral equations for the fields and the dyadic Green's functions

Defining the scattering potential $\overline{\mathbf{U}}_{\mathrm{c}}$ by

$\overline{\mathbf{U}}_{\mathrm{c}}\left(\mathbf{r}, \mathbf{r}^{\prime}\right)=\left(k_{V}^{2}-K^{2}\right) \delta\left(\mathbf{r}-\mathbf{r}^{\prime}\right) \overline{\mathbf{I}}=\overline{\mathbf{U}}\left(\mathbf{r}, \mathbf{r}^{\prime}\right)-\left(K^{2}-k_{1}^{2}\right) \delta\left(\mathbf{r}-\mathbf{r}^{\prime}\right) \overline{\mathbf{I}}$,

where $\overline{\mathbf{U}}\left(\mathbf{r}, \mathbf{r}^{\prime}\right)=\sum_{i} \overline{\mathbf{U}}_{i}\left(\mathbf{r}, \mathbf{r}^{\prime}\right), \overline{\mathbf{U}}_{i}\left(\mathbf{r}, \mathbf{r}^{\prime}\right)=U_{i}(\mathbf{r}) \delta\left(\mathbf{r}-\mathbf{r}^{\prime}\right) \overline{\mathbf{I}}$, and $U_{i}(\mathbf{r})=$ $k_{1}^{2}\left(\mathrm{~m}^{2}-1\right) \Theta\left(\mathbf{r}-\mathbf{R}_{i}\right)$, it can be shown that [13]

1. the fields $\mathbf{E}^{0}, \mathbf{E}^{1}$, and $\mathbf{E}^{2}$ satisfying the system of integral equations

$$
\begin{aligned}
& \mathbf{E}^{0}=\mathbf{E}_{\mathrm{K}}^{0}+\overline{\mathbf{G}}_{\mathrm{K}}^{01} \overline{\mathbf{U}}_{\mathrm{c}} \mathbf{E}^{1}, \\
& \mathbf{E}^{1}=\mathbf{E}_{\mathrm{K}}^{1}+\overline{\mathbf{G}}_{\mathrm{K}}^{11} \overline{\mathbf{U}}_{\mathrm{c}} \mathbf{E}^{1}, \\
& \mathbf{E}^{2}=\mathbf{E}_{\mathrm{K}}^{2}+\overline{\mathbf{G}}_{\mathrm{K}}^{21} \overline{\mathbf{U}}_{\mathrm{c}} \mathbf{E}^{1},
\end{aligned}
$$

also solve the boundary-value problem (6)-(12), and

2. the dyadic Green's functions $\overline{\mathbf{G}}^{01}, \overline{\mathbf{G}}^{11}$, and $\overline{\mathbf{G}}^{21}$ satisfying the system of integral equations

$\overline{\mathbf{G}}^{01}=\overline{\mathbf{G}}_{\mathrm{K}}^{01}+\overline{\mathbf{G}}_{\mathrm{K}}^{01} \overline{\mathbf{U}}_{\mathrm{c}} \overline{\mathbf{G}}^{11}$,

$\overline{\mathbf{G}}^{11}=\overline{\mathbf{G}}_{\mathrm{K}}^{11}+\overline{\mathbf{G}}_{\mathrm{K}}^{11} \overline{\mathbf{U}}_{\mathrm{c}} \overline{\mathbf{G}}^{11}$,

$$
\overline{\mathbf{G}}^{21}=\overline{\mathbf{G}}_{\mathrm{K}}^{21}+\overline{\mathbf{G}}_{\mathrm{K}}^{21} \overline{\mathbf{U}}_{\mathrm{c}} \overline{\mathbf{G}}^{11},
$$

also solve the boundary-value problem (13)-(19).

To prove for example Eq. (7) we apply the operator $\nabla \times \nabla \times$ $-K^{2} \overline{\mathbf{I}}$ to Eq. (28); using $\nabla \times \nabla \times \overline{\mathbf{G}}_{\mathrm{K}}^{11}-K^{2} \overline{\mathbf{G}}_{\mathrm{K}}^{11}=\delta \overline{\mathbf{I}}$ together with $\overline{\mathbf{U}}_{\mathrm{c}}=\left(k_{V}^{2}-K^{2}\right) \delta \overline{\mathbf{I}}$, where $\delta \overline{\mathbf{I}}$ stands for $\delta\left(\mathbf{r}-\mathbf{r}^{\prime}\right) \overline{\mathbf{I}}$, we obtain

$$
\begin{aligned}
\nabla \times \nabla \times \mathbf{E}^{1}-K^{2} \mathbf{E}^{1} & =\nabla \times \nabla \times \mathbf{E}_{\mathrm{K}}^{1}-K^{2} \mathbf{E}_{\mathrm{K}}^{1} \\
& +\left(\nabla \times \nabla \times \overline{\mathbf{G}}_{\mathrm{K}}^{11}-K^{2} \overline{\mathbf{G}}_{\mathrm{K}}^{11}\right) \overline{\mathbf{U}}_{\mathrm{c}} \mathbf{E}^{1} \\
& =\overline{\mathbf{U}}_{\mathrm{c}} \mathbf{E}^{1} \\
& =\left(k_{V}^{2}-K^{2}\right) \mathbf{E}^{1} .
\end{aligned}
$$

Eqs. (27)-(29) and (30)-(32) show that if $\overline{\mathbf{G}}^{11}$ is the solution of the integral equation (31), and the fields and the dyadic Green's functions for a homogeneous layer with wavenumber $K$ are known, then the fields and the dyadic Green's functions for a discrete random layer can be readily computed.
Let us introduce the transition operator $\overline{\mathbf{T}}_{\mathrm{c}}$ as the solution of the Lippmann-Schwinger equation

$$
\overline{\mathbf{T}}_{\mathrm{c}}=\overline{\mathbf{U}}_{\mathrm{c}}+\overline{\mathbf{U}}_{\mathrm{c}} \overline{\mathbf{G}}_{\mathrm{K}}^{11} \overline{\mathbf{T}}_{\mathrm{c}} .
$$

Considering the iterated solution of the Lippmann-Schwinger Eq. (34),

$\overline{\mathbf{T}}_{\mathrm{c}}=\overline{\mathbf{U}}_{\mathrm{c}}+\overline{\mathbf{U}}_{\mathrm{c}} \overline{\mathbf{G}}_{\mathrm{K}}^{11} \overline{\mathbf{U}}_{\mathrm{c}}+\cdots$,

and the iterated solution of the integral equation (cf. Eq. (31)) $\overline{\mathbf{G}}^{11}=\overline{\mathbf{G}}_{\mathrm{K}}^{11}+\overline{\mathbf{G}}_{\mathrm{K}}^{11} \overline{\mathbf{U}}_{\mathrm{c}} \overline{\mathbf{G}}^{11}$,

$$
\begin{aligned}
\overline{\mathbf{G}}^{11} & =\overline{\mathbf{G}}_{\mathrm{K}}^{11}+\overline{\mathbf{G}}_{\mathrm{K}}^{11} \overline{\mathbf{U}}_{\mathrm{c}} \overline{\mathbf{G}}_{\mathrm{K}}^{11}+\overline{\mathbf{G}}_{\mathrm{K}}^{11} \overline{\mathbf{U}}_{\mathrm{c}} \overline{\mathbf{G}}_{\mathrm{K}}^{11} \overline{\mathbf{U}}_{\mathrm{c}} \overline{\mathbf{G}}_{\mathrm{K}}^{11}+\cdots \\
& =\overline{\mathbf{G}}_{\mathrm{K}}^{11}+\overline{\mathbf{G}}_{\mathrm{K}}^{11}\left(\overline{\mathbf{U}}_{\mathrm{c}}+\overline{\mathbf{U}}_{\mathrm{c}} \overline{\mathbf{G}}_{\mathrm{K}}^{11} \overline{\mathbf{U}}_{\mathrm{c}}+\cdots\right) \overline{\mathbf{G}}_{\mathrm{K}}^{11},
\end{aligned}
$$

we find,

$\overline{\mathbf{G}}^{11}=\overline{\mathbf{G}}_{\mathrm{K}}^{11}+\overline{\mathbf{G}}_{\mathrm{K}}^{11} \overline{\mathbf{T}}_{\mathrm{c}} \overline{\mathbf{G}}_{\mathrm{K}}^{11}$.

By means of the series expansions (35) and (36) it can be shown that $\overline{\mathbf{U}}_{\mathrm{c}} \overline{\mathbf{G}}_{\mathrm{K}}^{11} \overline{\mathbf{T}}_{\mathrm{c}}=\overline{\mathbf{T}}_{\mathrm{c}} \overline{\mathbf{G}}_{\mathrm{K}}^{11} \overline{\mathbf{U}}_{\mathrm{c}}$; an equivalent representation of Eq. (34) is then

$\overline{\mathbf{T}}_{\mathrm{c}}=\overline{\mathbf{U}}_{\mathrm{c}}+\overline{\mathbf{T}}_{\mathrm{c}} \overline{\mathbf{G}}_{\mathrm{K}}^{11} \overline{\mathbf{U}}_{\mathrm{c}}$.

From Eqs. (31) and (37), we get

$\overline{\mathbf{T}}_{\mathrm{c}} \overline{\mathbf{G}}_{\mathrm{K}}^{11}=\overline{\mathbf{U}}_{\mathrm{c}} \overline{\mathbf{G}}^{11}$,

in which case, Eq. (38) becomes

$\overline{\mathbf{T}}_{\mathrm{c}}=\overline{\mathbf{U}}_{\mathrm{c}}+\overline{\mathbf{U}}_{\mathrm{c}} \overline{\mathbf{G}}_{\mathrm{K}}^{11} \overline{\mathbf{U}}_{\mathrm{c}}$.

Likewise, considering the iterated solution of the integral equation (cf. Eq. (28)) $\mathbf{E}^{1}=\mathbf{E}_{\mathrm{K}}^{1}+\overline{\mathbf{G}}_{\mathrm{K}}^{11} \overline{\mathbf{U}}_{\mathrm{c}} \mathbf{E}^{1}$ and using Eq. (35), we obtain

$\mathbf{E}^{1}=\mathbf{E}_{\mathrm{K}}^{1}+\overline{\mathbf{G}}_{\mathrm{K}}^{11} \overline{\mathbf{T}}_{\mathrm{c}} \mathbf{E}_{\mathrm{K}}^{1}$,

so that the relation

$\overline{\mathbf{T}}_{\mathrm{c}} \mathbf{E}_{\mathrm{K}}^{1}=\overline{\mathbf{U}}_{\mathrm{c}} \mathbf{E}^{1}$

readily follows. Inserting Eq. (42) in Eqs. (27)-(29) gives

$\mathbf{E}^{0}=\mathbf{E}_{\mathrm{K}}^{0}+\overline{\mathbf{G}}_{\mathrm{K}}^{01} \overline{\mathbf{T}}_{\mathrm{c}} \mathbf{E}_{\mathrm{K}}^{1}$,

$\mathbf{E}^{1}=\mathbf{E}_{\mathrm{K}}^{1}+\overline{\mathbf{G}}_{\mathrm{K}}^{11} \overline{\mathbf{T}}_{\mathrm{c}} \mathbf{E}_{\mathrm{K}}^{1}$

$\mathbf{E}^{2}=\mathbf{E}_{\mathrm{K}}^{2}+\overline{\mathbf{G}}_{\mathrm{K}}^{21} \overline{\mathbf{T}}_{\mathrm{c}} \mathbf{E}_{\mathrm{K}}^{1}$

while inserting Eq. (39) in Eqs. (30)-(32) yields

$\overline{\mathbf{G}}^{01}=\overline{\mathbf{G}}_{\mathrm{K}}^{01}+\overline{\mathbf{G}}_{\mathrm{K}}^{01} \overline{\mathbf{T}}_{\mathrm{c}} \overline{\mathbf{G}}_{\mathrm{K}}^{11}$,

$\overline{\mathbf{G}}^{11}=\overline{\mathbf{G}}_{\mathrm{K}}^{11}+\overline{\mathbf{G}}_{\mathrm{K}}^{11} \overline{\mathbf{T}}_{\mathrm{c}} \overline{\mathbf{G}}_{\mathrm{K}}^{11}$,

$\overline{\mathbf{G}}^{21}=\overline{\mathbf{G}}_{\mathrm{K}}^{21}+\overline{\mathbf{G}}_{\mathrm{K}}^{21} \overline{\mathbf{T}}_{\mathrm{c}} \overline{\mathbf{G}}_{\mathrm{K}}^{11}$.

From Eqs. (43)-(48), we see that all the scattering processes in the discrete random medium are described by the transition operator $\overline{\mathbf{T}}_{\mathrm{c}}$ [13]. 


\subsection{Coherent field and average dyadic Green's function}

To compute the configuration averages of the fields and the dyadic Green's functions we impose the effective field approximation:

$\left\langle\overline{\mathbf{G}}^{11}\right\rangle_{V}=\overline{\mathbf{G}}_{\mathrm{K}}^{11}$,

in which case, from Eq. (47), we obtain

$\left\langle\overline{\mathbf{T}}_{\mathrm{c}}\right\rangle_{V}=0$.

Taking the configuration average of Eqs. (43)-(45), and using Eq. (50) we obtain

$\left\langle\mathbf{E}^{0}\right\rangle_{V}=\mathbf{E}_{\mathrm{K}}^{0},\left\langle\mathbf{E}^{1}\right\rangle_{V}=\mathbf{E}_{\mathrm{K}}^{1},\left\langle\mathbf{E}^{2}\right\rangle_{V}=\mathbf{E}_{\mathrm{K}}^{2}$,

while taking the configuration average of Eqs. (46) and (48), and using again Eq. (50) we obtain

$\left\langle\overline{\mathbf{G}}^{01}\right\rangle_{V}=\overline{\mathbf{G}}_{\mathrm{K}}^{01},\left\langle\overline{\mathbf{G}}^{21}\right\rangle_{V}=\overline{\mathbf{G}}_{\mathrm{K}}^{21}$.

Thus, under the effective field approximation, the configuration averages of the fields and the dyadic Green's functions are the fields and the dyadic Green's functions, respectively, corresponding to a homogeneous layer with wavenumber $K$. Therefore, and in view of Eqs. (49) and (51), the coherent field $\left\langle\mathbf{E}^{1}\right\rangle_{V}$ and the average dyadic Green's function $\left\langle\overline{\mathbf{G}}^{11}\right\rangle_{V}$ are computed as follows.

1. For the incident plane electromagnetic wave as in Eq. (5), the coherent field in the domain $D_{1}$ is a superposition of up-going and down-going waves

$\left\langle\mathbf{E}^{1}(\mathbf{r})\right\rangle_{V}=\mathbf{E}_{\mathrm{K}}^{1}(\mathbf{r})=\sum_{a= \pm} \mathbf{E}_{\mathrm{c}}^{1 a}(\mathbf{r})$,

with

$\mathbf{E}_{c}^{1 a}(\mathbf{r})=\int \mathrm{e}^{\mathrm{j} \mathbf{k}_{1 \perp} \cdot \mathbf{r}_{\perp}} \mathrm{e}^{\mathrm{j} a K_{z}\left(\mathbf{k}_{1 \perp}\right) z} \overline{\mathbf{S}}^{1 a 0+}\left(\mathbf{k}_{1 \perp}, \mathbf{k}_{0 \mathrm{~s} \perp}\right) \cdot \mathcal{E}_{0}(\widehat{\mathbf{s}}) \mathrm{d}^{2} \mathbf{k}_{1 \perp}$

and

$K_{\mathrm{z}}\left(\mathbf{k}_{1 \perp}\right)=\sqrt{K^{2}-k_{1 \perp}^{2}}, \quad k_{1 \perp}=\left|\mathbf{k}_{1 \perp}\right|$.

2. The average dyadic Green's function $\left\langle\overline{\mathbf{G}}^{11}\right\rangle_{V}$ is given by

$$
\left\langle\overline{\mathbf{G}}^{11}\left(\mathbf{r}, \mathbf{r}^{\prime}\right)\right\rangle_{V}=\overline{\mathbf{G}}_{\mathrm{K}}^{11}\left(\mathbf{r}, \mathbf{r}^{\prime}\right)=\overline{\mathbf{G}}_{\mathrm{K}}\left(\mathbf{r}, \mathbf{r}^{\prime}\right)+\sum_{a, b= \pm} \overline{\mathbf{G}}_{\mathrm{K}}^{1 a 1 b}\left(\mathbf{r}, \mathbf{r}^{\prime}\right) .
$$

In Eq. (56), the dyadic Green's function for an unbounded medium $\overline{\mathbf{G}}_{\mathrm{K}}$ is

$\overline{\mathbf{G}}_{\mathrm{K}}\left(\mathbf{r}, \mathbf{r}_{1}\right)=\left\{\begin{array}{ll}\overline{\mathbf{G}}_{\mathrm{K}}^{+}\left(\mathbf{r}, \mathbf{r}_{1}\right) & z>z_{1}, \\ \overline{\mathbf{G}}_{\mathrm{K}}^{-}\left(\mathbf{r}, \mathbf{r}_{1}\right) & z<z_{1},\end{array}=\sum_{a= \pm} \overline{\mathbf{G}}_{\mathrm{K}}^{a}\left(\mathbf{r}, \mathbf{r}_{1}\right) \delta_{a, \operatorname{sgn}\left(z-z_{1}\right)}\right.$,

with

$\overline{\mathbf{G}}_{\mathrm{K}}^{a}\left(\mathbf{r}, \mathbf{r}_{1}\right)=\frac{\mathrm{j}}{8 \pi^{2}} \int \mathrm{e}^{\mathrm{j} \mathbf{k}_{1 \perp} \cdot\left(\mathbf{r}_{\perp}-\mathbf{r}_{1 \perp}\right)} \mathrm{e}^{\mathrm{j} a K_{\mathrm{z}}\left(\mathbf{k}_{1 \perp}\right)\left(z-z_{1}\right)} \overline{\mathbf{I}}_{\perp}^{a}\left(\mathbf{k}_{1 \perp}\right) \frac{\mathrm{d}^{2} \mathbf{k}_{1 \perp}}{K_{\mathrm{z}}\left(\mathbf{k}_{1 \perp}\right)}$,

$\overline{\mathbf{I}}_{\perp}^{a}\left(\mathbf{k}_{1 \perp}\right)=\sum_{\eta=\varphi, \theta} \widehat{\boldsymbol{\eta}}\left(\widehat{\mathbf{k}}_{1}^{a}\right) \otimes \widehat{\boldsymbol{\eta}}\left(\widehat{\mathbf{k}}_{1}^{a}\right)$

and

$\delta_{a, \operatorname{sgn}\left(z-z_{1}\right)}=\left\{\begin{array}{ll}1, & a=\operatorname{sgn}\left(z-z_{1}\right) \\ 0, & a \neq \operatorname{sgn}\left(z-z_{1}\right)\end{array}\right.$,

while the dyadic Green's function $\overline{\mathbf{G}}_{\mathrm{K}}^{1 a 1 b}$ describing the reflections at the boundaries is

$\overline{\mathbf{G}}_{\mathrm{K}}^{1 a 1 b}\left(\mathbf{r}, \mathbf{r}_{1}\right)=\frac{\mathrm{j}}{8 \pi^{2}} \int \mathrm{e}^{\mathrm{j}\left(\mathbf{k}_{\perp} \cdot \mathbf{r}_{\perp}-\mathbf{k}_{1 \perp} \cdot \mathbf{r}_{1 \perp}\right)} \mathrm{e}^{\mathrm{j}\left[a K_{z}\left(\mathbf{k}_{\perp}\right) z-b K_{\mathrm{z}}\left(\mathbf{k}_{1 \perp}\right) z_{1}\right]}$

$$
\times \overline{\mathbf{S}}^{1 a 1 b}\left(\mathbf{k}_{\perp}, \mathbf{k}_{1 \perp}\right) \frac{\mathrm{d}^{2} \mathbf{k}_{\perp} \mathrm{d}^{2} \mathbf{k}_{1 \perp}}{K_{\mathrm{z}}\left(\mathbf{k}_{1 \perp}\right)}
$$

with

$\overline{\mathbf{S}}^{1 a 1 b}\left(\mathbf{k}_{\perp}, \mathbf{k}_{1 \perp}\right)=\sum_{\eta, \mu=\varphi, \theta}\left[\overline{\mathbf{S}}^{1 a 1 b}\left(\mathbf{k}_{\perp}, \mathbf{k}_{1 \perp}\right)\right]_{\eta \mu} \widehat{\boldsymbol{\eta}}\left(\widehat{\mathbf{k}}^{a}\right) \otimes \widehat{\boldsymbol{\mu}}\left(\widehat{\mathbf{k}}_{1}^{b}\right)$.

In Eq. (62), the unit vector $\widehat{\mathbf{k}}^{a}$, which enters the expressions for $\widehat{\boldsymbol{\theta}}\left(\widehat{\mathbf{k}}^{a}\right)$ and $\widehat{\boldsymbol{\varphi}}\left(\widehat{\mathbf{k}}^{a}\right)$, is computed as

$\widehat{\mathbf{k}}^{a}=\frac{\mathbf{k}^{a}}{\left|\mathbf{k}^{a}\right|}, \quad \mathbf{k}^{a}=\mathbf{k}_{\perp}+a K_{\mathbf{z}}^{\prime}\left(\mathbf{k}_{\perp}\right) \widehat{\mathbf{z}}, \quad a= \pm$,

where

$K_{\mathrm{z}}^{\prime}=\operatorname{Re}\left(K_{\mathrm{z}}\right)$ and $K_{\mathrm{z}}^{\prime \prime}=\operatorname{Im}\left(K_{\mathrm{z}}\right)$.

Similarly, the unit vector $\widehat{\mathbf{k}}_{1}^{a}$ is computed as $\widehat{\mathbf{k}}^{a}=\mathbf{k}_{1}^{a} /\left|\mathbf{k}_{1}^{a}\right|$ with $\mathbf{k}_{1}^{a}=\mathbf{k}_{1 \perp}+a K_{\mathbf{z}}^{\prime}\left(\mathbf{k}_{1 \perp}\right) \widehat{\mathbf{z}}$. Strictly speaking the expression of $\mathbf{k}^{a}$ should involve $K_{\mathrm{z}}$ and not its real part $K_{\mathrm{z}}^{\prime}$. The approximation (63) implies that the unit vectors $\widehat{\boldsymbol{\theta}}\left(\widehat{\mathbf{k}}^{a}\right)$ and $\widehat{\boldsymbol{\varphi}}\left(\widehat{\mathbf{k}}^{a}\right)$ are real, and relies on the assumption that the contribution of evanescent waves in the plane wave representation (61) is much smaller than the contribution of propagating waves $[10,11]$. The neglect of the evanescent waves which decay far from the sources and boundaries, but can be significant in the near field, is equivalent to the far-field approximation for the fields. At the present moment, we use this approximation only when computing the unit vectors $\widehat{\boldsymbol{\theta}}\left(\widehat{\mathbf{k}}^{a}\right)$ and $\widehat{\boldsymbol{\varphi}}\left(\widehat{\mathbf{k}}^{a}\right)$.

Some properties of the scattering operators are summarized below $[13,15]$.

1. In Eqs. (54) and (61), the scattering operators $\overline{\mathbf{S}}^{100+}$ and $\overline{\mathbf{S}}^{1 a 1 b}$ correspond to a homogeneous layer with the effective wavenumber $K$, and so they are averaged over volumetric fluctuations. They can be computed by using the operators $\overline{\mathbf{R}}_{01}$, $\overline{\mathbf{T}}_{10}$, and $\overline{\mathbf{R}}_{H 21}$, which describe the scattering by the lower and the upper rough surface. Explicitly, we have

$$
\begin{aligned}
& \overline{\mathbf{S}}^{1+1+}=\left(\overline{\mathbf{I}}_{\perp}^{1+1+}-\overline{\mathbf{R}}_{01} \overline{\mathbf{R}}_{H 21}\right)^{-1} \overline{\mathbf{R}}_{01} \overline{\mathbf{R}}_{H 21}=\overline{\mathbf{R}}_{01} \overline{\mathbf{S}}^{1-1+}, \\
& \overline{\mathbf{S}}^{1+1-}=\left(\overline{\mathbf{I}}_{\perp}^{1+1+}-\overline{\mathbf{R}}_{01} \overline{\mathbf{R}}_{H 21}\right)^{-1} \overline{\mathbf{R}}_{01}=\overline{\mathbf{R}}_{01}\left(\overline{\mathbf{I}}_{\perp}^{1-1-}+\overline{\mathbf{S}}^{1-1-}\right), \\
& \overline{\mathbf{S}}^{1-1+}=\overline{\mathbf{R}}_{H 21}\left(\overline{\mathbf{I}}_{\perp}^{1+1+}-\overline{\mathbf{R}}_{01} \overline{\mathbf{R}}_{H 21}\right)^{-1}=\overline{\mathbf{R}}_{H 21}\left(\overline{\mathbf{I}}_{\perp}^{1+1+}+\overline{\mathbf{S}}^{1+1+}\right), \\
& \overline{\mathbf{S}}^{1-1-}=\overline{\mathbf{R}}_{H 21}\left(\overline{\mathbf{I}}_{\perp}^{1+1+}-\overline{\mathbf{R}}_{01} \overline{\mathbf{R}}_{H 21}\right)^{-1} \overline{\mathbf{R}}_{01}=\overline{\mathbf{R}}_{H 21} \overline{\mathbf{S}}^{1+1-}, \\
& \overline{\mathbf{S}}^{1+0+}=\left(\overline{\mathbf{I}}_{\perp}^{1+1+}-\overline{\mathbf{R}}_{01} \overline{\mathbf{R}}_{H 21}\right)^{-1} \overline{\mathbf{T}}_{10}, \\
& \overline{\mathbf{S}}^{1-0+}=\overline{\mathbf{R}}_{H 21} \overline{\mathbf{S}}^{1+0+},
\end{aligned}
$$

where the dyadic projector $\overline{\mathbf{I}}_{\perp}^{1 a 1 b}$,

$\overline{\mathbf{I}}_{\perp}^{1 a 1 b}\left(\mathbf{k}_{\perp}, \mathbf{k}_{1 \perp}\right)=\delta\left(\mathbf{k}_{\perp}-\mathbf{k}_{1 \perp}\right)\left[\widehat{\boldsymbol{\varphi}}\left(\widehat{\mathbf{k}}^{a}\right) \otimes \widehat{\boldsymbol{\varphi}}\left(\widehat{\mathbf{k}}^{b}\right)+\widehat{\boldsymbol{\theta}}\left(\widehat{\mathbf{k}}^{a}\right) \otimes \widehat{\boldsymbol{\theta}}\left(\widehat{\mathbf{k}}^{b}\right)\right]$,

is the linear identity mapping from the space vector defined by the basis $\left(\widehat{\boldsymbol{\varphi}}\left(\widehat{\mathbf{k}}^{b}\right), \widehat{\boldsymbol{\theta}}\left(\widehat{\mathbf{k}}^{b}\right)\right)$ onto the space vector defined by the basis $\left(\widehat{\boldsymbol{\varphi}}\left(\widehat{\mathbf{k}}^{a}\right), \widehat{\boldsymbol{\theta}}\left(\widehat{\mathbf{k}}^{a}\right)\right)$. Also note the relation connecting the reflection operators corresponding to a rough surface centered at the plane $z=H$ and the plane $z=0$,

$\overline{\mathbf{R}}_{H 21}\left(\mathbf{k}_{\perp}, \mathbf{k}_{1 \perp}\right)=\mathrm{e}^{\mathrm{j}\left[K_{z}\left(\mathbf{k}_{\perp}\right)+K_{\mathrm{z}}\left(\mathbf{k}_{1 \perp}\right)\right] H} \overline{\mathbf{R}}_{21}\left(\mathbf{k}_{\perp}, \mathbf{k}_{1 \perp}\right)$. 
2. Any surface-averaged scattering dyadic operator is of the form

$\left\langle\overline{\mathbf{S}}\left(\mathbf{p}_{\perp}, \mathbf{p}_{1 \perp}\right)\right\rangle_{S}=\delta\left(\mathbf{p}_{\perp}-\mathbf{p}_{1 \perp}\right) \overline{\mathcal{S}}\left(\mathbf{p}_{\perp}\right)$,

while any surface-averaged dyadic correlation function of a scattering operator is of the form

$\left\langle\overline{\mathbf{S}}\left(\mathbf{p}_{\perp}+\frac{\mathbf{P}_{\perp}}{2}, \mathbf{p}_{1 \perp}+\frac{\mathbf{P}_{1 \perp}}{2}\right) \otimes \overline{\mathbf{S}}^{\star}\left(\mathbf{p}_{\perp}-\frac{\mathbf{P}_{\perp}}{2}, \mathbf{p}_{1 \perp}-\frac{\mathbf{P}_{1 \perp}}{2}\right)\right\rangle_{S}$

$=\delta\left(\mathbf{P}_{\perp}-\mathbf{P}_{1 \perp}\right) \overline{\overline{\mathcal{S}}}\left(\mathbf{p}_{\perp}, \mathbf{p}_{1 \perp}, \mathbf{P}_{\perp}\right)$.

If the illuminated surface has a finite area $A$, then $\overline{\overline{\mathcal{S}}}\left(\mathbf{p}_{\perp}, \mathbf{p}_{1 \perp}, \mathbf{P}_{\perp}\right)$ is given by

$\overline{\overline{\mathcal{S}}}\left(\mathbf{p}_{\perp}, \mathbf{p}_{1 \perp}, \mathbf{P}_{\perp}\right)$

$=\frac{(2 \pi)^{2}}{A}\left\langle\overline{\mathbf{S}}\left(\mathbf{p}_{\perp}+\frac{\mathbf{P}_{\perp}}{2}, \mathbf{p}_{1 \perp}+\frac{\mathbf{P}_{\perp}}{2}\right) \otimes \overline{\mathbf{S}}^{\star}\left(\mathbf{p}_{\perp}-\frac{\mathbf{P}_{\perp}}{2}, \mathbf{p}_{1 \perp}-\frac{\mathbf{P}_{\perp}}{2}\right)\right\rangle_{S}$

so that in the case $\mathbf{P}_{\perp}=0$, we have

$$
\overline{\overline{\mathcal{S}}}\left(\mathbf{p}_{\perp}, \mathbf{p}_{1 \perp}, 0\right)=\frac{(2 \pi)^{2}}{A}\left\langle\overline{\mathbf{S}}\left(\mathbf{p}_{\perp}, \mathbf{p}_{1 \perp}\right) \otimes \overline{\mathbf{S}}^{\star}\left(\mathbf{p}_{\perp}, \mathbf{p}_{1 \perp}\right)\right\rangle_{S} .
$$

To compute the effective wavenumber $K$ we make the approximation $\overline{\mathbf{G}}_{\mathrm{K}}^{11} \approx \overline{\mathbf{G}}_{\mathrm{K}}$. In this case, the effective wavenumber is that of an unbounded discrete random medium, and in the framework of the effective field approximation, $K$ is computed as in Ref. [11]. Considering the representation of the dyadic Green's function for a layer of discrete scatterers (cf. Eq. (56)) $\overline{\mathbf{G}}_{\mathrm{K}}^{11}=\overline{\mathbf{G}}_{\mathrm{K}}+\sum_{a, b= \pm} \overline{\mathbf{G}}_{\mathrm{K}}^{1 a 1 b}$, it is clear that the approximation $\overline{\mathbf{G}}_{\mathrm{K}}^{11} \approx \overline{\mathbf{G}}_{\mathrm{K}}$ can be made if we neglect all boundary effects. For example, from Eq. (61), we see that $\overline{\mathbf{G}}_{\mathrm{K}}^{1+1+}$ is expressed in terms of $\overline{\mathbf{S}}^{1+1+}$, which in view of Eqs. (65) and (72), contains the factor

$\mathrm{e}^{\mathrm{j}\left[K_{\mathbf{z}}\left(\mathbf{k}_{\perp}\right)+K_{\mathbf{z}}\left(\mathbf{k}_{1 \perp}\right)\right] H}=\mathrm{e}^{\mathrm{j}\left[K_{\mathbf{z}}^{\prime}\left(\mathbf{k}_{\perp}\right)+K_{\mathbf{z}}^{\prime}\left(\mathbf{k}_{1 \perp}\right)\right] H} \mathrm{e}^{-\left[K_{\mathrm{z}}^{\prime \prime}\left(\mathbf{k}_{\perp}\right)+K_{\mathrm{z}}^{\prime \prime}\left(\mathbf{k}_{1 \perp}\right)\right] H}$.

This factor is negligible far away from the upper boundary when the condition $K_{\mathrm{z}}^{\prime \prime} H \gg 1$ is satisfied, or equivalently, when the thickness of the layer $H$ is much larger than the mean free path $l_{\mathrm{mp}}$.

\subsection{Integral equation for the dyadic correlation function}

The dyadic correlation function satisfies the Bethe-Salpeter equation

$\left\langle\mathbf{E}^{1}(\mathbf{r}) \otimes \mathbf{E}^{1 \star}\left(\mathbf{r}^{\prime}\right)\right\rangle_{V}$

$=\left\langle\mathbf{E}^{1}(\mathbf{r})\right\rangle_{V} \otimes\left\langle\mathbf{E}^{1 \star}\left(\mathbf{r}^{\prime}\right)\right\rangle_{V}+\int_{D_{1}}\left\langle\overline{\mathbf{G}}^{11}\left(\mathbf{r}, \mathbf{r}_{1}\right)\right\rangle_{V} \otimes\left\langle\overline{\mathbf{G}}^{11 \star}\left(\mathbf{r}^{\prime}, \mathbf{r}_{1}^{\prime}\right)\right\rangle_{V}$

$\cdot \overline{\overline{\mathbf{I}}}\left(\mathbf{r}_{1}, \mathbf{r}_{2}, \mathbf{r}_{1}^{\prime}, \mathbf{r}_{2}^{\prime}\right) \cdot\left\langle\mathbf{E}^{1}\left(\mathbf{r}_{2}\right) \otimes \mathbf{E}^{1 \star}\left(\mathbf{r}_{2}^{\prime}\right)\right\rangle_{V} \mathrm{~d}^{3} \mathbf{r}_{1}^{\prime} \mathrm{d}^{3} \mathbf{r}_{2}^{\prime} \mathrm{d}^{3} \mathbf{r}_{1} \mathrm{~d}^{3} \mathbf{r}_{2}$.

In the ladder approximation for the Bethe-Salpeter equation, the (tetradic) scattering intensity operator in Eq. (77) is given by [2]

$\overline{\overline{\mathbf{I}}}\left(\mathbf{r}, \mathbf{r}_{1}, \mathbf{r}^{\prime}, \mathbf{r}_{1}^{\prime}\right)=n_{0} \int_{D_{1}} \overline{\mathbf{T}}_{i}\left(\mathbf{r}, \mathbf{r}_{1}\right) \otimes \overline{\mathbf{T}}_{i}^{\star}\left(\mathbf{r}^{\prime}, \mathbf{r}_{1}^{\prime}\right) \mathrm{d}^{3} \mathbf{R}_{i}$,

where $\overline{\mathbf{T}}_{i}\left(\mathbf{r}, \mathbf{r}_{1}\right)$ is the transition operator for the $i$ th particle centered at $\mathbf{R}_{i}$.

Let us decompose the total field into a coherent and an incoherent component

$\mathbf{E}^{1}(\mathbf{r})=\left\langle\mathbf{E}^{1}(\mathbf{r})\right\rangle_{V}+\mathscr{E}^{1}(\mathbf{r})$,

denote the dyadic correlation function and its diffuse and coherent components by $\overline{\mathbf{C}}, \overline{\mathscr{C}}$ and $\overline{\mathbf{C}}_{\mathrm{c}}$, respectively, i.e.,

$\overline{\mathbf{C}}\left(\mathbf{r}, \mathbf{r}^{\prime}\right)=\left\langle\mathbf{E}^{1}(\mathbf{r}) \otimes \mathbf{E}^{1 \star}\left(\mathbf{r}^{\prime}\right)\right\rangle_{V}$,

$\overline{\mathscr{C}}\left(\mathbf{r}, \mathbf{r}^{\prime}\right)=\left\langle\mathscr{E}^{1}(\mathbf{r}) \otimes \mathscr{E}^{1 \star}\left(\mathbf{r}^{\prime}\right)\right\rangle_{V}$,
$\overline{\mathbf{C}}_{\mathrm{c}}\left(\mathbf{r}, \mathbf{r}^{\prime}\right)=\left\langle\mathbf{E}^{1}(\mathbf{r})\right\rangle_{V} \otimes\left\langle\mathbf{E}^{1 \star}\left(\mathbf{r}^{\prime}\right)\right\rangle_{V}$.

and note the following relationship between the dyadic correlation functions:

$\overline{\mathbf{C}}\left(\mathbf{r}, \mathbf{r}^{\prime}\right)=\overline{\mathbf{C}}_{\mathrm{c}}\left(\mathbf{r}, \mathbf{r}^{\prime}\right)+\overline{\mathscr{C}}\left(\mathbf{r}, \mathbf{r}^{\prime}\right)$.

Further on, defining the tetradic Green's function by

$\overline{\overline{\mathbf{G}}}\left(\mathbf{r}, \mathbf{r}_{1}, \mathbf{r}^{\prime}, \mathbf{r}_{1}^{\prime}\right)=\left\langle\overline{\mathbf{G}}^{11}\left(\mathbf{r}, \mathbf{r}_{1}\right)\right\rangle_{V} \otimes\left\langle\overline{\mathbf{G}}^{11 \star}\left(\mathbf{r}^{\prime}, \mathbf{r}_{1}^{\prime}\right)\right\rangle_{V}$,

we express Eq. (77) as

$$
\begin{gathered}
\overline{\mathscr{C}}\left(\mathbf{r}, \mathbf{r}^{\prime}\right)=\int_{D_{1}} \overline{\overline{\mathbf{G}}}\left(\mathbf{r}, \mathbf{r}_{1}, \mathbf{r}^{\prime}, \mathbf{r}_{1}^{\prime}\right) \cdot \overline{\overline{\mathbf{I}}}\left(\mathbf{r}_{1}, \mathbf{r}_{2}, \mathbf{r}_{1}^{\prime}, \mathbf{r}_{2}^{\prime}\right) \\
\cdot \overline{\mathbf{C}}\left(\mathbf{r}_{2}, \mathbf{r}_{2}^{\prime}\right) \mathrm{d}^{3} \mathbf{r}_{1}^{\prime} \mathrm{d}^{3} \mathbf{r}_{2}^{\prime} \mathrm{d}^{3} \mathbf{r}_{1} \mathrm{~d}^{3} \mathbf{r}_{2} .
\end{gathered}
$$

Taking the average over surface fluctuations, and employing the weak surface correlation approximation, according to which, $\overline{\overline{\mathbf{G}}}$ and $\overline{\mathbf{C}}$ are assumed to be weakly correlated on the rough surfaces, we obtain

$$
\begin{gathered}
\left\langle\overline{\mathscr{C}}\left(\mathbf{r}, \mathbf{r}^{\prime}\right)\right\rangle_{S}=\int_{D_{1}}\left\langle\overline{\overline{\mathbf{G}}}\left(\mathbf{r}, \mathbf{r}_{1}, \mathbf{r}^{\prime}, \mathbf{r}_{1}^{\prime}\right)\right\rangle_{S} \cdot \overline{\overline{\mathbf{I}}}\left(\mathbf{r}_{1}, \mathbf{r}_{2}, \mathbf{r}_{1}^{\prime}, \mathbf{r}_{2}^{\prime}\right) \\
\cdot\left\langle\overline{\mathbf{C}}\left(\mathbf{r}_{2}, \mathbf{r}_{2}^{\prime}\right)\right\rangle_{S} \mathrm{~d}^{3} \mathbf{r}_{1}^{\prime} \mathrm{d}^{3} \mathbf{r}_{2}^{\prime} \mathrm{d}^{3} \mathbf{r}_{1} \mathrm{~d}^{3} \mathbf{r}_{2},
\end{gathered}
$$

where

$\left\langle\overline{\mathbf{C}}\left(\mathbf{r}, \mathbf{r}^{\prime}\right)\right\rangle_{S}=\left\langle\overline{\mathbf{C}}_{\mathrm{c}}\left(\mathbf{r}, \mathbf{r}^{\prime}\right)\right\rangle_{S}+\left\langle\overline{\mathscr{C}}\left(\mathbf{r}, \mathbf{r}^{\prime}\right)\right\rangle_{S}$,

$\left\langle\overline{\mathbf{C}}_{\mathrm{c}}\left(\mathbf{r}, \mathbf{r}^{\prime}\right)\right\rangle_{S}=\left\langle\left\langle\mathbf{E}^{1}(\mathbf{r})\right\rangle_{V} \otimes\left\langle\mathbf{E}^{1 \star}\left(\mathbf{r}^{\prime}\right)\right\rangle_{V}\right\rangle_{S}$,

and

$\left\langle\overline{\overline{\mathbf{G}}}\left(\mathbf{r}, \mathbf{r}_{1}, \mathbf{r}^{\prime}, \mathbf{r}_{1}^{\prime}\right)\right\rangle_{S}=\left\langle\left\langle\overline{\mathbf{G}}^{11}\left(\mathbf{r}, \mathbf{r}_{1}\right)\right\rangle_{V} \otimes\left\langle\overline{\mathbf{G}}^{11 \star}\left(\mathbf{r}^{\prime}, \mathbf{r}_{1}^{\prime}\right)\right\rangle_{V}\right\rangle_{S}$,

The next step is to apply the Wigner and the two-dimensional Fourier transform to the integral equation (86).

Wigner transform. The Wigner transform of a dyadic $\overline{\mathbf{X}}\left(\mathbf{r}, \mathbf{r}^{\prime}\right)$ is defined by

$\overline{\mathbf{X}}_{\mathrm{W}}\left(\frac{\mathbf{r}+\mathbf{r}^{\prime}}{2}, \mathbf{p}\right)=\int \overline{\mathbf{X}}\left(\mathbf{r}, \mathbf{r}^{\prime}\right) \mathrm{e}^{-\mathrm{j} \mathbf{p} \cdot\left(\mathbf{r}-\mathbf{r}^{\prime}\right)} \mathrm{d}^{3}\left(\mathbf{r}-\mathbf{r}^{\prime}\right)$,

and the inverse transform is

$\overline{\mathbf{X}}\left(\mathbf{r}, \mathbf{r}^{\prime}\right)=\frac{1}{(2 \pi)^{3}} \int \overline{\mathbf{X}}_{\mathrm{W}}\left(\frac{\mathbf{r}+\mathbf{r}^{\prime}}{2}, \mathbf{p}\right) \mathrm{e}^{\mathrm{j} \mathbf{p} \cdot\left(\mathbf{r}-\mathbf{r}^{\prime}\right)} \mathrm{d}^{3} \mathbf{p}$.

For a tetradic $\overline{\overline{\mathbf{X}}}\left(\mathbf{r}, \mathbf{r}_{1}, \mathbf{r}^{\prime}, \mathbf{r}_{1}^{\prime}\right)$, the direct and inverse Wigner transforms are given, respectively, by

$$
\begin{aligned}
\overline{\overline{\mathbf{X}}}_{\mathrm{W}}\left(\frac{\mathbf{r}+\mathbf{r}^{\prime}}{2}, \mathbf{p}, \frac{\mathbf{r}_{1}+\mathbf{r}_{1}^{\prime}}{2}, \mathbf{p}_{1}\right) & =\int \mathrm{e}^{-\mathrm{j} \mathbf{p} \cdot\left(\mathbf{r}-\mathbf{r}^{\prime}\right)} \overline{\overline{\mathbf{X}}}\left(\mathbf{r}, \mathbf{r}_{1}, \mathbf{r}^{\prime}, \mathbf{r}_{1}^{\prime}\right) \\
& \times \mathrm{e}^{\mathrm{j} \mathbf{p}_{1} \cdot\left(\mathbf{r}_{1}-\mathbf{r}_{1}^{\prime}\right)} \mathrm{d}^{3}\left(\mathbf{r}_{1}-\mathbf{r}_{1}^{\prime}\right) \mathrm{d}^{3}\left(\mathbf{r}-\mathbf{r}^{\prime}\right),
\end{aligned}
$$

and

$$
\begin{aligned}
\overline{\overline{\mathbf{X}}}\left(\mathbf{r}, \mathbf{r}_{1}, \mathbf{r}^{\prime}, \mathbf{r}_{1}^{\prime}\right) & =\frac{1}{(2 \pi)^{6}} \int \mathrm{e}^{\mathrm{j} \mathbf{p} \cdot\left(\mathbf{r}-\mathbf{r}^{\prime}\right)} \overline{\bar{X}}_{\mathrm{w}}\left(\frac{\mathbf{r}+\mathbf{r}^{\prime}}{2}, \mathbf{p}, \frac{\mathbf{r}_{1}+\mathbf{r}_{1}^{\prime}}{2}, \mathbf{p}_{1}\right) \\
& \times \mathrm{e}^{-\mathrm{j} \mathbf{p}_{1} \cdot\left(\mathbf{r}_{1}-\mathbf{r}_{1}^{\prime}\right)} \mathrm{d}^{3} \mathbf{p}_{1} \mathrm{~d}^{3} \mathbf{p} .
\end{aligned}
$$

Applying the Wigner transform to the integral equation (86) under the assumption that for a layer with a large geometrical thickness $H$, we can approximate

$$
\begin{aligned}
\int_{D_{1}} \mathrm{~d}^{3}\left(\mathbf{r}-\mathbf{r}^{\prime}\right) & =\int_{-H}^{H}\left[\int \mathrm{d}^{2}\left(\mathbf{r}_{\perp}-\mathbf{r}_{\perp}^{\prime}\right)\right] \mathrm{d}\left(z-z^{\prime}\right) \\
& \approx \iint \mathrm{d}^{2}\left(\mathbf{r}_{\perp}-\mathbf{r}_{\perp}^{\prime}\right) \mathrm{d}\left(z-z^{\prime}\right),
\end{aligned}
$$


we obtain

$$
\begin{aligned}
\overline{\mathscr{C}}_{\mathrm{W}}(\mathbf{R}, \mathbf{p})= & \frac{1}{(2 \pi)^{6}} \int_{D_{1}}\left[\int \overline{\overline{\mathbf{G}}}_{\mathrm{W}}\left(\mathbf{R}, \mathbf{p}, \mathbf{R}_{1}, \mathbf{p}_{1}\right) \cdot \overline{\overline{\mathbf{I}}}_{\mathrm{W}}\left(\mathbf{R}_{1}, \mathbf{p}_{1}, \mathbf{R}_{2}, \mathbf{p}_{2}\right)\right. \\
& \left.\cdot \overline{\mathbf{C}}_{\mathrm{W}}\left(\mathbf{R}_{2}, \mathbf{p}_{2}\right) \mathrm{d}^{3} \mathbf{p}_{1} \mathrm{~d}^{3} \mathbf{p}_{2}\right] \mathrm{d}^{3} \mathbf{R}_{1} \mathrm{~d}^{3} \mathbf{R}_{2},
\end{aligned}
$$

where $\mathbf{R}=\left(\mathbf{r}+\mathbf{r}^{\prime}\right) / 2, \mathbf{R}_{1}=\left(\mathbf{r}_{1}+\mathbf{r}_{1}^{\prime}\right) / 2$, and $\overline{\mathscr{C}}_{\mathrm{W}}, \overline{\mathbf{C}}_{\mathrm{W}}, \overline{\overline{\mathbf{G}}}_{\mathrm{W}}$, and $\overline{\overline{\mathbf{I}}}_{\mathrm{W}}$ are the Wigner transforms of $\langle\overline{\mathscr{C}}\rangle_{S},\langle\overline{\mathbf{C}}\rangle_{S},\langle\overline{\overline{\mathbf{G}}}\rangle_{S}$, and $\overline{\overline{\mathbf{I}}}$, respectively, that is,

$\langle\overline{\mathscr{C}}\rangle_{S} \stackrel{\mathcal{W}}{\longmapsto} \overline{\mathscr{C}}_{\mathrm{W}},\langle\overline{\mathbf{C}}\rangle_{S} \stackrel{\mathcal{W}}{\longmapsto} \overline{\mathbf{C}}_{\mathrm{W}},\langle\overline{\overline{\mathbf{G}}}\rangle_{S} \stackrel{\mathcal{W}}{\longmapsto} \overline{\overline{\mathbf{G}}}_{\mathrm{w}}$, and $\overline{\overline{\mathbf{I}}} \stackrel{\mathcal{W}}{\longmapsto} \overline{\mathbf{I}}_{\mathrm{W}}$.

Note that in the Wigner-transform space, Eq. (87) becomes

$\overline{\mathbf{C}}_{\mathrm{W}}(\mathbf{R}, \mathbf{p})=\overline{\mathbf{C}}_{\mathrm{cW}}(\mathbf{R}, \mathbf{p})+\overline{\mathscr{C}}_{\mathrm{W}}(\mathbf{R}, \mathbf{p})$.

If the problem has translational invariance in azimuth, the Wigner transform of the dyadic $\overline{\mathbf{X}}\left(\mathbf{r}, \mathbf{r}^{\prime}\right)$, where $\overline{\mathbf{X}}$ stands for $\langle\overline{\mathscr{C}}\rangle_{S},\left\langle\overline{\mathbf{C}}_{\mathrm{c}}\right\rangle_{S}$, or $\langle\overline{\mathbf{C}}\rangle_{S}$, is of the form

$\overline{\mathbf{X}}_{\mathrm{W}}(\mathbf{R}, \mathbf{p})=\overline{\mathbf{X}}_{\mathrm{W}}(Z, \mathbf{p})$,

while the Wigner transform of the tetradic $\overline{\bar{X}}\left(\mathbf{r}, \mathbf{r}_{1}, \mathbf{r}^{\prime}, \mathbf{r}_{1}^{\prime}\right)$, where $\overline{\overline{\mathbf{X}}}$ stands for $\langle\overline{\overline{\mathbf{G}}}\rangle_{S}$ or $\overline{\overline{\mathbf{I}}}$, is of the form

$\overline{\mathbf{X}}_{\mathrm{W}}\left(\mathbf{R}, \mathbf{p}, \mathbf{R}_{1}, \mathbf{p}_{1}\right)=\overline{\overline{\mathbf{X}}}_{\mathrm{W}}\left(\mathbf{R}_{\perp}-\mathbf{R}_{1 \perp}, Z, \mathbf{p}, Z_{1}, \mathbf{p}_{1}\right)$.

Here, $\mathbf{R}=\mathbf{R}_{\perp}+Z \widehat{\mathbf{z}}$ and $\mathbf{R}_{1}=\mathbf{R}_{1 \perp}+Z_{1} \widehat{\mathbf{z}}$, and clearly, $Z=(z+$ $\left.z^{\prime}\right) / 2$ and $Z_{1}=\left(z_{1}+z_{1}^{\prime}\right) / 2$.

Fourier transform. The two-dimensional Fourier transform of a dyadic $\overline{\mathbf{X}}_{\mathrm{W}}(\mathbf{R}, \mathbf{p})=\overline{\mathbf{X}}_{\mathrm{W}}\left(\mathbf{R}_{\perp}, Z, \mathbf{p}\right)$ along the horizontal variable $\mathbf{R}_{\perp}$ is defined by

$\overline{\mathbf{X}}_{\mathrm{WF}}\left(Z, \mathbf{p}, \mathbf{P}_{\perp}\right)=\int \mathrm{e}^{-\mathrm{j} \mathbf{R}_{\perp} \cdot \mathbf{P}_{\perp}} \overline{\mathbf{X}}_{\mathrm{W}}\left(\mathbf{R}_{\perp}, Z, \mathbf{p}\right) \mathrm{d}^{2} \mathbf{R}_{\perp} ;$

the inverse transform is

$\overline{\mathbf{X}}_{\mathrm{W}}\left(\mathbf{R}_{\perp}, Z, \mathbf{p}\right)=\frac{1}{(2 \pi)^{2}} \int \mathrm{e}^{\mathrm{j} \mathbf{R}_{\perp} \cdot \mathbf{P}_{\perp}} \overline{\mathbf{X}}_{\mathrm{WF}}\left(Z, \mathbf{p}, \mathbf{P}_{\perp}\right) \mathrm{d}^{2} \mathbf{P}_{\perp}$.

For a dyadic $\overline{\mathbf{X}}_{\mathrm{W}}(\mathbf{R}, \mathbf{p})$ satisfying Eq. (96), we have

$\overline{\mathbf{X}}_{\mathrm{WF}}\left(Z, \mathbf{p}, \mathbf{P}_{\perp}\right)=(2 \pi)^{2} \delta\left(\mathbf{P}_{\perp}\right) \overline{\mathbf{X}}_{\mathrm{W}}(Z, \mathbf{p})$,

while for a tetradic $\overline{\overline{\mathbf{X}}}_{\mathrm{W}}\left(\mathbf{R}, \mathbf{p}, \mathbf{R}_{1}, \mathbf{p}_{1}\right)$ satisfying Eq. (97), the direct and inverse two-dimensional Fourier transforms along the horizontal variable $\mathbf{R}_{\perp}-\mathbf{R}_{1}$ are given, respectively, by

$$
\begin{aligned}
& \overline{\overline{\mathbf{X}}}_{\mathrm{WF}}\left(Z, \mathbf{p}, Z_{1}, \mathbf{p}_{1}, \mathbf{P}_{\perp}\right)=\int \mathrm{e}^{-\mathrm{j}\left(\mathbf{R}_{\perp}-\mathbf{R}_{1 \perp}\right) \cdot \mathbf{P}_{\perp}} \\
& \quad \times \overline{\overline{\mathbf{X}}}_{\mathrm{W}}\left(\mathbf{R}_{\perp}-\mathbf{R}_{1 \perp}, Z, \mathbf{p}, Z_{1}, \mathbf{p}_{1}\right) \mathrm{d}^{2}\left(\mathbf{R}_{\perp}-\mathbf{R}_{1 \perp}\right) \\
& \text { and } \\
& \overline{\overline{\mathbf{X}}}_{\mathrm{W}}\left(\mathbf{R}_{\perp}-\mathbf{R}_{1 \perp}, Z, \mathbf{p}, Z_{1}, \mathbf{p}_{1}\right)=\frac{1}{(2 \pi)^{2}} \int \mathrm{e}^{\mathrm{j}\left(\mathbf{R}_{\perp}-\mathbf{R}_{1 \perp}\right) \cdot \mathbf{P}_{\perp}} \\
& \quad \times \overline{\mathbf{X}}_{\mathrm{WF}}\left(Z, \mathbf{p}, Z_{1}, \mathbf{p}_{1}, \mathbf{P}_{\perp}\right) \mathrm{d}^{2} \mathbf{P}_{\perp} .
\end{aligned}
$$

Applying the two-dimensional Fourier transform to the integral equation (94), taking into account that the problem has translational invariance in azimuth (the two-dimensional Fourier transforms of $\overline{\mathscr{C}}_{\mathrm{W}}$ and $\overline{\mathbf{C}}_{\mathrm{W}}$ are as in Eq. (100)), and using the identity $\delta\left(\mathbf{P}_{\perp}\right) f\left(\mathbf{P}_{\perp}\right)=\delta\left(\mathbf{P}_{\perp}\right) f(0)$, we obtain

$$
\begin{aligned}
\overline{\mathscr{C}}_{\mathrm{W}}(Z, \mathbf{p})= & \frac{1}{(2 \pi)^{6}} \int_{0}^{H}\left[\int \overline{\overline{\mathbf{G}}}_{\mathrm{WF}}\left(Z, \mathbf{p}, Z_{1}, \mathbf{p}_{1}, 0\right)\right. \\
& \cdot \overline{\overline{\mathbf{I}}}_{\mathrm{WF}}\left(Z_{1}, \mathbf{p}_{1}, Z_{2}, \mathbf{p}_{2}, 0\right) \\
& \left.\cdot \overline{\mathbf{C}}_{\mathrm{W}}\left(Z_{2}, \mathbf{p}_{2}\right) \mathrm{d}^{3} \mathbf{p}_{1} \mathrm{~d}^{3} \mathbf{p}_{2}\right] \mathrm{d} Z_{1} \mathrm{~d} Z_{2},
\end{aligned}
$$

where $\overline{\overline{\mathbf{G}}}_{\mathrm{WF}}$ and $\overline{\overline{\mathbf{I}}}_{\mathrm{WF}}$ are the two-dimensional Fourier transforms of $\overline{\mathbf{G}}_{\mathrm{W}}$ and $\overline{\overline{\mathbf{I}}}_{\mathrm{W}}$, respectively, that is,

$\overline{\overline{\mathbf{G}}}_{\mathrm{W}} \stackrel{\mathcal{F}}{\longmapsto} \overline{\overline{\mathbf{G}}}_{\mathrm{WF}}$ and $\overline{\overline{\mathbf{I}}}_{\mathrm{W}} \stackrel{\mathcal{F}}{\longmapsto} \overline{\overline{\mathbf{I}}}_{\mathrm{WF}}$.
In the following we will use the term Wigner-Fourier transform to designate the successive Wigner and Fourier transforms, that is,

$\overline{\mathbf{X}} \stackrel{\mathcal{W}}{\longmapsto} \overline{\mathbf{X}}_{\mathrm{W}} \stackrel{\mathcal{F}}{\longmapsto} \overline{\mathbf{X}}_{\mathrm{WF}} \stackrel{\text { def }}{=} \overline{\mathbf{X}} \stackrel{\mathcal{W F}}{\longmapsto} \overline{\mathbf{X}}_{\mathrm{WF}}$,

where $\overline{\mathbf{X}}$ stands for $\langle\overline{\overline{\mathbf{G}}}\rangle_{S}$ or $\overline{\overline{\mathbf{I}}}$. The integral equation (103) is the starting point of our analysis. For its solution we must determine the Wigner transforms $\overline{\mathbf{C}}_{\mathrm{W}}(Z, \mathbf{p})$ and $\overline{\mathscr{C}}_{\mathrm{W}}(Z, \mathbf{p})$, as well as the Wigner-Fourier transforms $\overline{\overline{\mathbf{G}}}_{\mathrm{WF}}\left(Z, \mathbf{p}, Z_{1}, \mathbf{p}_{1}, \mathbf{P}_{\perp}\right)$ and $\overline{\overline{\mathbf{I}}}_{\mathrm{WF}}\left(Z, \mathbf{p}, Z_{1}, \mathbf{p}_{1}, \mathbf{P}_{\perp}\right)$ at $\mathbf{P}_{\perp}=0$.

\subsection{Wigner and Wigner-Fourier transforms of the basic quantities}

In this section we evaluate the Wigner and Wigner-Fourier transforms of the basic quantities entering in the integral equation (103).

\subsubsection{Tetradic Green's function}

When computing the Wigner-Fourier transform of the tetradic Green's function $\overline{\overline{\mathbf{G}}}\left(\mathbf{r}, \mathbf{r}_{1}, \mathbf{r}^{\prime}, \mathbf{r}_{1}^{\prime}\right)$, we exclude all cross terms. In fact, taking into account that between two scattering events on the rough surface the wave interacts with the particles, we assume that the scattering events on the rough surface are uncorrelated [14,16]. Thus, using (cf. Eqs. (56), (57), and (84))

$$
\begin{aligned}
\overline{\overline{\mathbf{G}}}\left(\mathbf{r}, \mathbf{r}_{1}, \mathbf{r}^{\prime}, \mathbf{r}_{1}^{\prime}\right)= & \left\langle\overline{\mathbf{G}}^{11}\left(\mathbf{r}, \mathbf{r}_{1}\right)\right\rangle_{V} \otimes\left\langle\overline{\mathbf{G}}^{11 \star}\left(\mathbf{r}^{\prime}, \mathbf{r}_{1}^{\prime}\right)\right\rangle_{V} \\
\approx & \sum_{a= \pm} \overline{\mathbf{G}}_{\mathrm{K}}^{a}\left(\mathbf{r}, \mathbf{r}_{1}\right) \otimes \overline{\mathbf{G}}_{\mathrm{K}}^{a \star}\left(\mathbf{r}^{\prime}, \mathbf{r}_{1}^{\prime}\right) \delta_{a, \operatorname{sgn}\left(z-z_{1}\right)} \delta_{a, \operatorname{sgn}\left(z^{\prime}-z_{1}^{\prime}\right)} \\
& +\sum_{a, b= \pm} \overline{\mathbf{G}}_{\mathrm{K}}^{1 a 1 b}\left(\mathbf{r}, \mathbf{r}_{1}\right) \otimes \overline{\mathbf{G}}_{\mathrm{K}}^{1 a 1 b_{\star}}\left(\mathbf{r}^{\prime}, \mathbf{r}_{1}^{\prime}\right)
\end{aligned}
$$

and noting that

$$
\begin{aligned}
& \sum_{a= \pm} \overline{\mathbf{G}}_{\mathrm{K}}^{a}\left(\mathbf{r}, \mathbf{r}_{1}\right) \otimes \overline{\mathbf{G}}_{\mathrm{K}}^{a \star}\left(\mathbf{r}^{\prime}, \mathbf{r}_{1}^{\prime}\right) \delta_{a, \operatorname{sgn}\left(z-z_{1}\right)} \delta_{a, \operatorname{sgn}\left(z^{\prime}-z_{1}^{\prime}\right)} \\
& =\sum_{a= \pm} \overline{\mathbf{G}}_{\mathrm{K}}^{a}\left(\mathbf{r}, \mathbf{r}_{1}\right) \otimes \overline{\mathbf{G}}_{\mathrm{K}}^{a_{\star}}\left(\mathbf{r}^{\prime}, \mathbf{r}_{1}^{\prime}\right) \delta_{a, \operatorname{sgn}\left(Z-Z_{1}\right)},
\end{aligned}
$$

we obtain the Wigner-Fourier transform at $\mathbf{P}_{\perp}=0$ :

$$
\begin{aligned}
\overline{\overline{\mathbf{G}}}_{\mathrm{WF}}\left(Z, \mathbf{p}, Z_{1}, \mathbf{p}_{1}, 0\right)= & \sum_{a, b= \pm}\left[\overline{\overline{\mathbf{G}}}_{\mathrm{KWF}}^{a b}\left(Z, \mathbf{p}, Z_{1}, \mathbf{p}_{1}, 0\right) \delta_{a b} \delta_{a, \operatorname{sgn}\left(Z-Z_{1}\right)}\right. \\
& \left.+\overline{\overline{\mathbf{G}}}_{\mathrm{KWF}}^{1 a 1 b}\left(Z, \mathbf{p}, Z_{1}, \mathbf{p}_{1}, 0\right)\right]
\end{aligned}
$$

where

$$
\begin{aligned}
\overline{\overline{\mathbf{G}}}_{\mathrm{KWF}}^{a b}\left(Z, \mathbf{p}, Z_{1}, \mathbf{p}_{1}, 0\right)= & \frac{\left(2 \pi^{2}\right)^{2}}{\left|K_{\mathbf{z}}\left(\mathbf{p}_{1 \perp}\right)\right|^{2}} \mathrm{e}^{-2 a K_{\mathrm{z}}^{\prime \prime}\left(\mathbf{p}_{\perp}\right) Z} \mathrm{e}^{2 b K_{\mathrm{z}}^{\prime \prime}\left(\mathbf{p}_{1}\right) Z_{1}} \\
& \times \delta\left(\mathbf{p}_{\perp}-\mathbf{p}_{1 \perp}\right) \delta\left(p_{\mathrm{z}}-a K_{\mathrm{z}}^{\prime}\left(\mathbf{p}_{\perp}\right)\right) \\
& \times \delta\left(p_{1 \mathrm{z}}-b K_{\mathrm{z}}^{\prime}\left(\mathbf{p}_{1 \perp}\right)\right) \overline{\overline{\mathbf{I}}}_{\perp}^{a a}\left(\mathbf{p}_{\perp}\right)
\end{aligned}
$$

$\overline{\overline{\mathbf{I}}}_{\perp}^{a a}\left(\mathbf{p}_{\perp}\right)=\overline{\mathbf{I}}_{\perp}^{a}\left(\mathbf{p}_{\perp}\right) \otimes \overline{\mathbf{I}}_{\perp}^{a}\left(\mathbf{p}_{\perp}\right)$,

$\overline{\mathbf{I}}_{\perp}^{a}\left(\mathbf{p}_{\perp}\right)=\sum_{\eta=\varphi, \theta} \widehat{\boldsymbol{\eta}}\left(\widehat{\mathbf{p}}^{a}\right) \otimes \widehat{\boldsymbol{\eta}}\left(\widehat{\mathbf{p}}^{a}\right)$,

and

$$
\begin{aligned}
\overline{\overline{\mathbf{G}}}_{\mathrm{KWF}}^{1 a 1 b}\left(Z, \mathbf{p}, Z_{1}, \mathbf{p}_{1}, 0\right)= & \frac{\left(2 \pi^{2}\right)^{2}}{\left|K_{\mathrm{z}}\left(\mathbf{p}_{1 \perp}\right)\right|^{2}} \mathrm{e}^{-2 a K_{\mathrm{z}}^{\prime \prime}\left(\mathbf{p}_{\perp}\right) Z} \mathrm{e}^{2 b K_{\mathrm{z}}^{\prime \prime}\left(\mathbf{p}_{1 \perp}\right) Z_{1}} \\
& \times \delta\left(p_{\mathrm{z}}-a K_{\mathrm{z}}^{\prime}\left(\mathbf{p}_{\perp}\right)\right) \delta\left(p_{1 \mathrm{z}}-b K_{\mathrm{z}}^{\prime}\left(\mathbf{p}_{1 \perp}\right)\right) \\
& \times \overline{\overline{\mathcal{S}}}^{1 a 1 b}\left(\mathbf{p}_{\perp}, \mathbf{p}_{1 \perp}, 0\right) .
\end{aligned}
$$


The tetradic $\overline{\overline{\mathcal{S}}}^{1 a 1 b}\left(\mathbf{p}_{\perp}, \mathbf{p}_{1 \perp}, 0\right)$ in Eq. (110), possessing the dyadicproduct representation

$$
\begin{aligned}
\overline{\overline{\mathcal{S}}}^{1 a 1 b}\left(\mathbf{p}_{\perp}, \mathbf{p}_{1 \perp}, 0\right)= & \sum_{\eta, \mu, \eta^{\prime}, \mu^{\prime}=\varphi, \theta}\left[\overline{\overline{\mathcal{S}}}^{1 a 1 b}\left(\mathbf{p}_{\perp}, \mathbf{p}_{1 \perp}, 0\right)\right]_{\eta \mu \eta^{\prime} \mu^{\prime}} \\
& \times \widehat{\boldsymbol{\eta}}\left(\widehat{\mathbf{p}}^{a}\right) \otimes \widehat{\boldsymbol{\mu}}\left(\widehat{\mathbf{p}}_{1}^{b}\right) \otimes \widehat{\boldsymbol{\eta}}^{\prime}\left(\widehat{\mathbf{p}}^{a}\right) \otimes \widehat{\boldsymbol{\mu}}^{\prime}\left(\widehat{\mathbf{p}}_{1}^{b}\right),
\end{aligned}
$$

is defined by (cf. Eq. (76))

$\overline{\mathcal{\mathcal { S }}}^{1 a 1 b}\left(\mathbf{p}_{\perp}, \mathbf{p}_{1 \perp}, 0\right)=\frac{(2 \pi)^{2}}{A}\left\langle\overline{\mathbf{S}}^{1 a 1 b}\left(\mathbf{p}_{\perp}, \mathbf{p}_{1 \perp}\right) \otimes \overline{\mathbf{S}}^{1 a 1 b_{\star}}\left(\mathbf{p}_{\perp}, \mathbf{p}_{1 \perp}\right)\right\rangle_{S}$.

Note that during the derivation, the following representation for the Wigner transform is obtained:

$\overline{\overline{\mathbf{G}}}_{\mathrm{W}}\left(\mathbf{R}, \mathbf{p}, \mathbf{R}_{1}, \mathbf{p}_{1}\right)=\overline{\overline{\mathbf{G}}}_{\mathrm{W}}\left(\mathbf{R}_{\perp}-\mathbf{R}_{1 \perp}, Z, \mathbf{p}, Z_{1}, \mathbf{p}_{1}\right) ;$

hence, $\overline{\overline{\mathbf{G}}}_{\mathrm{W}}\left(\mathbf{R}, \mathbf{p}, \mathbf{R}_{1}, \mathbf{p}_{1}\right)$ has the property (97), which reflects the translational invariance in azimuth.

\subsubsection{Scattering intensity operator}

Considering the integral representation of the scattering intensity operator as given by Eq. (78) and using ( $\overline{\mathbf{T}}_{i}$ is a translational dyadic, i.e., $\overline{\mathbf{T}}_{i}\left(\mathbf{r}, \mathbf{r}^{\prime}\right)=\overline{\mathbf{T}}\left(\mathbf{r}-\mathbf{R}_{i}, \mathbf{r}^{\prime}-\mathbf{R}_{i}\right)$ )

$\overline{\mathbf{T}}_{i p}\left(\mathbf{p}, \mathbf{p}^{\prime}\right)=\mathrm{e}^{-\mathrm{j} \mathbf{p} \cdot \mathbf{R}_{i}} \overline{\mathbf{T}}_{p}\left(\mathbf{p}, \mathbf{p}^{\prime}\right) \mathrm{e}^{\mathrm{j} \mathbf{p}^{\prime} \cdot \mathbf{R}_{i}}$,

where $\overline{\mathbf{T}}_{i p}\left(\mathbf{p}, \mathbf{p}^{\prime}\right)$ is the Fourier transform of the transition operator of particle $i$ centered at $\mathbf{R}_{i}$, and $\overline{\mathbf{T}}_{p}\left(\mathbf{p}, \mathbf{p}^{\prime}\right)$ is that of a particle centered at the origin of the coordinate system [11], we find that the Wigner transform of $\overline{\overline{\mathbf{I}}}\left(\mathbf{r}, \mathbf{r}_{1}, \mathbf{r}^{\prime}, \mathbf{r}_{1}^{\prime}\right)$ is

$$
\begin{aligned}
\overline{\overline{\mathbf{I}}}_{\mathrm{W}}\left(\mathbf{R}, \mathbf{p}, \mathbf{R}_{1}, \mathbf{p}_{1}\right)= & \frac{n_{0}}{(2 \pi)^{3}} \int \mathrm{e}^{\mathrm{j} \mathbf{P} \cdot\left(\mathbf{R}-\mathbf{R}_{1}\right)} \overline{\mathbf{T}}_{p}\left(\mathbf{p}+\frac{\mathbf{P}}{2}, \mathbf{p}_{1}+\frac{\mathbf{P}}{2}\right) \\
& \otimes \overline{\mathbf{T}}_{p}^{\star}\left(\mathbf{p}-\frac{\mathbf{P}}{2}, \mathbf{p}_{1}-\frac{\mathbf{P}}{2}\right) \mathrm{d}^{3} \mathbf{P} .
\end{aligned}
$$

By virtue of Eq. (115), the tetradic $\overline{\overline{\mathbf{I}}}_{W}\left(\mathbf{R}, \mathbf{p}, \mathbf{R}_{1}, \mathbf{p}_{1}\right)$ has the property (97), which states the translational invariance in azimuth. Furthermore, the two-dimensional Fourier transform of $\overline{\overline{\mathbf{I}}}_{\mathrm{W}}\left(\mathbf{R}, \mathbf{p}, \mathbf{R}_{1}, \mathbf{p}_{1}\right)$ at $\mathbf{P}_{\perp}=0$ computes as

$$
\begin{aligned}
\overline{\overline{\mathbf{I}}}_{\mathrm{WF}}\left(Z, \mathbf{p}, Z_{1}, \mathbf{p}_{1}, 0\right)= & n_{0} \int \mathrm{e}^{\mathrm{j}\left(Z-Z_{1}\right) P_{1 \mathrm{z}}} \overline{\mathbf{T}}_{p}\left(\mathbf{p}+\frac{P_{1 \mathrm{z}} \widehat{\mathbf{z}}}{2}, \mathbf{p}_{1}+\frac{P_{1 \mathrm{z}} \widehat{\mathbf{z}}}{2}\right) \\
& \otimes \overline{\mathbf{T}}_{p}^{\star}\left(\mathbf{p}-\frac{P_{1 \mathrm{z}} \widehat{\mathbf{z}}}{2}, \mathbf{p}_{1}-\frac{P_{1 \mathrm{z}} \widehat{\mathbf{z}}}{2}\right) \mathrm{d} P_{1 \mathrm{z}} .
\end{aligned}
$$

The integral representation (116) shows that the operator $\overline{\overline{\mathbf{I}}}_{\mathrm{WF}}\left(Z, \mathbf{p}, Z_{1}, \mathbf{p}_{1}, 0\right)$ is non-local $\left(\overline{\overline{\mathbf{I}}}_{\mathrm{WF}} \neq 0\right.$ when $\left.Z \neq Z_{1}\right)$, and that the dyadic $\overline{\mathbf{T}}_{p}$ is off-shell evaluated, since in general

$\left|\mathbf{p}+a \frac{P_{1 \mathrm{z}} \widehat{\mathbf{z}}}{2}\right| \neq\left|\mathbf{p}_{1}+a \frac{P_{1 \mathrm{z}} \widehat{\mathbf{z}}}{2}\right|$.

To simplify the analysis, we use the on-shell approximation for the dyadic $\overline{\mathbf{T}}_{p}[14,17]$

$\overline{\mathbf{T}}_{p}\left(\mathbf{p}+a \frac{P_{1 \mathrm{z}} \widehat{\mathbf{z}}}{2}, \mathbf{p}_{1}+a \frac{P_{1 \mathbf{z}} \widehat{\mathbf{z}}}{2}\right) \approx \overline{\mathbf{T}}_{p}\left(\mathbf{p}^{\operatorname{sgn}\left(p_{\mathrm{z}}\right)}, \mathbf{p}_{1}^{\operatorname{sgn}\left(p_{1 \mathrm{z}}\right)}\right)$,

where for $\mathbf{p}=\mathbf{p}_{\perp}+p_{z} \widehat{\mathbf{z}}$ and $\mathbf{p}_{1}=\mathbf{p}_{1 \perp}+p_{1 \mathrm{z}} \widehat{\mathbf{z}}, \mathbf{p}^{\operatorname{sgn}\left(p_{\mathrm{z}}\right)}$ and $\mathbf{p}_{1}^{\operatorname{sgn}\left(p_{1 z}\right)}$ stand, respectively, for

$$
\begin{aligned}
\mathbf{p}^{\operatorname{sgn}\left(p_{\mathrm{z}}\right)} & =\mathbf{p}_{\perp}+\operatorname{sgn}\left(p_{\mathrm{z}}\right) K_{\mathrm{z}}^{\prime}\left(\mathbf{p}_{\perp}\right) \widehat{\mathbf{z}}, \\
\mathbf{p}_{1}^{\operatorname{sgn}\left(p_{1 \mathrm{z}}\right)} & =\mathbf{p}_{1 \perp}+\operatorname{sgn}\left(p_{1 \mathrm{z}}\right) K_{\mathrm{z}}^{\prime}\left(\mathbf{p}_{1 \perp}\right) \widehat{\mathbf{z}} .
\end{aligned}
$$

By virtue of Eq. (117), the operator $\overline{\overline{\mathbf{I}}}_{\mathrm{WF}}\left(Z, \mathbf{p}, Z_{1}, \mathbf{p}_{1}, 0\right)$ becomes localized $\left(\overline{\overline{\mathbf{I}}}_{\mathrm{WF}}=0\right.$ when $\left.Z \neq Z_{1}\right)$, and we have

$$
\overline{\overline{\mathbf{I}}}_{\mathrm{WF}}\left(Z, \mathbf{p}, Z_{1}, \mathbf{p}_{1}, 0\right)=n_{0} \delta\left(Z-Z_{1}\right) \overline{\mathbf{T}}_{p}\left(\mathbf{p}^{\operatorname{sgn}\left(p_{\mathrm{z}}\right)}, \mathbf{p}_{1}^{\operatorname{sgn}\left(p_{1 \mathbf{z}}\right)}\right)
$$

$$
\otimes \overline{\mathbf{T}}_{p}^{\star}\left(\mathbf{p}^{\operatorname{sgn}\left(p_{\mathbf{z}}\right)}, \mathbf{p}_{1}^{\operatorname{sgn}\left(p_{1 z}\right)}\right) .
$$

Moreover, because of $\left|\mathbf{p}^{\operatorname{sgn}\left(p_{z}\right)}\right|=\left|\mathbf{p}_{1}^{\operatorname{sgn}\left(p_{1 z}\right)}\right| \approx K^{\prime}$ for $K^{\prime \prime} \ll K^{\prime}$, the dyadic $\overline{\mathbf{T}}_{p}$ is on-shell evaluated.

\subsubsection{Coherent dyadic correlation function}

As in the case of the tetradic Green's function, the Wigner transform of the coherent dyadic correlation function is computed by neglecting the correlations between the up-going and downgoing coherent waves. Thus, by means of Eq. (53), we have

$\overline{\mathbf{C}}_{\mathrm{c}}\left(\mathbf{r}, \mathbf{r}^{\prime}\right)=\left\langle\mathbf{E}^{1}(\mathbf{r})\right\rangle_{V} \otimes\left\langle\mathbf{E}^{1 \star}\left(\mathbf{r}^{\prime}\right)\right\rangle_{V} \approx \sum_{a= \pm} \overline{\mathbf{C}}_{\mathrm{c}}^{a a}\left(\mathbf{r}, \mathbf{r}^{\prime}\right)$

$\overline{\mathbf{C}}_{\mathrm{c}}^{a a}\left(\mathbf{r}, \mathbf{r}^{\prime}\right)=\mathbf{E}_{\mathrm{c}}^{1 a}(\mathbf{r}) \otimes \mathbf{E}_{\mathrm{c}}^{1 a_{\star}}\left(\mathbf{r}^{\prime}\right)$.

Applying the Wigner transform to $\left\langle\overline{\mathbf{C}}_{\mathrm{c}}\right\rangle_{S}$, and using the dyadic identity

$(\overline{\mathbf{A}} \cdot \mathbf{c}) \otimes(\overline{\mathbf{B}} \cdot \mathbf{d})=(\overline{\mathbf{A}} \otimes \overline{\mathbf{B}}) \cdot(\mathbf{c} \otimes \mathbf{d})$,

we obtain

$\overline{\mathbf{C}}_{\mathrm{cW}}(\mathbf{R}, \mathbf{p})=\overline{\mathbf{C}}_{\mathrm{cW}}(Z, \mathbf{p})$,

with

$\overline{\mathbf{C}}_{\mathrm{cw}}(Z, \mathbf{p})=(2 \pi)^{3} \sum_{a= \pm} \delta\left(p_{\mathrm{z}}-a K_{\mathrm{z}}^{\prime}\left(\mathbf{p}_{\perp}\right)\right) \overline{\mathbf{C}}_{\mathrm{c}}^{a a}\left(Z, \mathbf{p}_{\perp}\right)$,

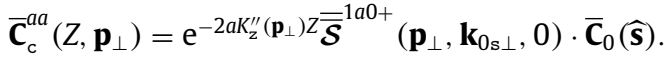

In agreement with Eq. (96), Eq. (122) shows that the problem is translationally invariant in azimuth. In Eq. (124), $\overline{\mathbf{C}}_{0}(\widehat{\mathbf{s}})=$ $\mathcal{E}_{0}(\widehat{\mathbf{s}}) \otimes \mathcal{E}_{0}^{\star}(\widehat{\mathbf{s}})$, and the tetradic $\overline{\overline{\mathcal{S}}}^{100+}\left(\mathbf{p}_{\perp}, \mathbf{k}_{0 \mathrm{~s} \perp}, 0\right)$ is defined by (cf. Eq. (76))

$$
\begin{aligned}
\overline{\overline{\mathcal{S}}}^{1 a+}\left(\mathbf{p}_{\perp}, \mathbf{k}_{0 \mathrm{~s} \perp}, 0\right) \\
=\frac{(2 \pi)^{2}}{A}\left\langle\overline{\mathbf{S}}^{1 a 0+}\left(\mathbf{p}_{\perp}, \mathbf{k}_{0 \mathrm{~s} \perp}\right) \otimes \overline{\mathbf{S}}^{1 a 0+\star}\left(\mathbf{p}_{\perp}, \mathbf{k}_{0 \mathrm{~s} \perp}\right)\right\rangle_{S} .
\end{aligned}
$$

Some properties of the dyadic $\overline{\mathbf{C}}_{\mathrm{c}}^{a a}\left(Z, \mathbf{p}_{\perp}\right)$ are listed below.

1. From Eqs. (124) and (125), we see that $\overline{\mathbf{C}}_{\mathrm{c}}^{a a}\left(Z, \mathbf{p}_{\perp}\right)$ is transverse, that is,

$$
\overline{\mathbf{C}}_{\mathrm{c}}^{a a}\left(Z, \mathbf{p}_{\perp}\right)=\sum_{\eta, \mu=\varphi, \theta}\left[\overline{\mathbf{C}}_{\mathrm{c}}^{a a}\left(Z, \mathbf{p}_{\perp}\right)\right]_{\eta \mu} \widehat{\boldsymbol{\eta}}\left(\widehat{\mathbf{p}}^{a}\right) \otimes \widehat{\boldsymbol{\mu}}\left(\widehat{\mathbf{p}}^{a}\right),
$$

with $\mathbf{p}^{a}=\mathbf{p}_{\perp}+a K_{\mathrm{z}}^{\prime}\left(\mathbf{p}_{\perp}\right) \widehat{\mathbf{z}}$.

2. The $Z$-dependence of $\overline{\mathbf{C}}_{c}^{a a}\left(Z, \mathbf{p}_{\perp}\right)$ can be determined explicitly. From Eqs. (124) and (125), together with Eqs. (69), (70), and (72), we find

$\overline{\mathbf{C}}_{\mathrm{c}}^{++}\left(Z, \mathbf{p}_{\perp}\right) \propto T_{+}(Z, H)=\mathrm{e}^{-2 K_{z}^{\prime \prime}\left(\mathbf{p}_{\perp}\right) Z}$,

and

$\overline{\mathbf{C}}_{\mathrm{c}}^{--}\left(Z, \mathbf{p}_{\perp}\right) \propto T_{-}(Z, H)=\mathrm{e}^{-2 K_{\mathrm{z}}^{\prime \prime}\left(\mathbf{k}_{0 \mathrm{~s} \perp}\right) H} \mathrm{e}^{-2 K_{\mathrm{z}}^{\prime \prime}\left(\mathbf{p}_{\perp}\right)(H-Z)}$.

The latter follows from

$\overline{\mathbf{S}}^{1-0+}\left(\mathbf{p}_{\perp}, \mathbf{k}_{0 \mathrm{~s} \perp}\right) \propto \mathrm{e}^{\mathrm{j}\left[K_{\mathrm{z}}\left(\mathbf{p}_{\perp}\right)+K_{\mathrm{z}}\left(\mathbf{k}_{0 \mathrm{~s} \perp}\right)\right] H}$,

which gives

$\left[\overline{\overline{\mathcal{S}}}^{1-0+}\left(\mathbf{p}_{\perp}, \mathbf{k}_{0 \mathrm{~s} \perp}, 0\right)\right]_{\eta \mu \eta^{\prime} \mu^{\prime}} \propto \mathrm{e}^{-2 K_{\mathrm{z}}^{\prime \prime}\left(\mathbf{p}_{\perp}\right) H} \mathrm{e}^{-2 K_{\mathrm{z}}^{\prime \prime}\left(\mathbf{k}_{0 \mathrm{~s} \perp}\right) H}$.

The transmission function $T_{+}(Z, H)$ describes the attenuation of the up-going coherent field through the medium, while the transmission function $T_{-}(Z, H)$ describes the attenuation of the down-going coherent field, which first propagates through the layer and is then reflected at the upper boundary. 
3. From Eq. (124), it follows that $\overline{\mathbf{C}}_{\mathrm{c}}^{a a}\left(Z, \mathbf{p}_{\perp}\right)$ satisfies the differential equation

$\frac{\partial}{\partial Z} \overline{\mathbf{C}}_{\mathrm{c}}^{a a}\left(Z, \mathbf{p}_{\perp}\right)=-2 a K_{\mathrm{z}}^{\prime \prime}\left(\mathbf{p}_{\perp}\right) \overline{\mathbf{C}}_{\mathrm{c}}^{a a}\left(Z, \mathbf{p}_{\perp}\right)$.

Setting $Z=H$ in Eq. (124) gives

$\overline{\mathbf{C}}_{\mathrm{c}}^{++}\left(H, \mathbf{p}_{\perp}\right)=\mathrm{e}^{-2 K_{\mathrm{z}}^{\prime \prime}\left(\mathbf{p}_{\perp}\right) H} \overline{\overline{\mathcal{S}}}^{1+0+}\left(\mathbf{p}_{\perp}, \mathbf{k}_{0 \mathrm{~s} \perp}, 0\right) \cdot \overline{\mathbf{C}}_{0}(\widehat{\mathbf{s}})$,

$$
\overline{\mathbf{C}}_{\mathrm{c}}^{--}\left(H, \mathbf{p}_{\perp}\right)=\mathrm{e}^{2 K_{\mathrm{z}}^{\prime \prime}\left(\mathbf{p}_{\perp}\right) H} \overline{\overline{\mathcal{S}}}^{1-0+}\left(\mathbf{p}_{\perp}, \mathbf{k}_{0 \mathrm{~s} \perp}, 0\right) \cdot \overline{\mathbf{C}}_{0}(\widehat{\mathbf{s}}) .
$$

Then, using Eqs. (70), (72), and (125) we find the boundary condition

$$
\overline{\mathbf{C}}_{\mathrm{c}}^{--}\left(H, \mathbf{p}_{\perp}\right)=\int \overline{\overline{\mathcal{R}}}_{21}\left(\mathbf{p}_{\perp}, \mathbf{p}_{1 \perp}, 0\right) \cdot \overline{\mathbf{C}}_{\mathrm{c}}^{++}\left(H, \mathbf{p}_{1 \perp}\right) \mathrm{d}^{2} \mathbf{p}_{1 \perp}
$$

at $Z=H$, where the tetradic reflection operator $\overline{\overline{\mathcal{R}}}_{21}\left(\mathbf{p}_{\perp}, \mathbf{p}_{1 \perp}, 0\right)$ is defined by

$$
\overline{\overline{\mathcal{R}}}_{21}\left(\mathbf{p}_{\perp}, \mathbf{p}_{1 \perp}, 0\right)=\frac{(2 \pi)^{2}}{A}\left\langle\overline{\mathbf{R}}_{21}\left(\mathbf{p}_{\perp}, \mathbf{p}_{1 \perp}\right) \otimes \overline{\mathbf{R}}_{21}^{\star}\left(\mathbf{p}_{\perp}, \mathbf{p}_{1 \perp}\right)\right\rangle_{S} .
$$

\subsubsection{Diffuse dyadic correlation function}

The diffuse dyadic correlation function is the key quantity in our analysis and for this reason it deserves more attention. First, we observe that the tetradic $\overline{\overline{\mathbf{G}}}_{\mathrm{WF}}$ can be written as

$$
\begin{aligned}
\overline{\overline{\mathbf{G}}}_{\mathrm{WF}}\left(Z, \mathbf{p}, Z_{1}, \mathbf{p}_{1}, 0\right)= & (2 \pi)^{4} \sum_{a= \pm} \delta\left(p_{\mathrm{z}}-a K_{\mathrm{z}}^{\prime}\left(\mathbf{p}_{\perp}\right)\right) \\
& \times \overline{\overline{\mathcal{G}}}^{a a}\left(Z, \mathbf{p}_{\perp}, Z_{1}, \mathbf{p}_{1}, 0\right),
\end{aligned}
$$

where the expression of $\overline{\overline{\mathcal{G}}}^{a a}\left(Z, \mathbf{p}_{\perp}, Z_{1}, \mathbf{p}_{1}, 0\right)$ follows from Eqs. (106), (107), and (110). Inserting Eq. (136) in Eq. (103), we infer that $\overline{\mathscr{C}}_{\mathrm{W}}(Z, \mathbf{p})$ can be expressed as

$\overline{\mathscr{C}}_{\mathrm{W}}(Z, \mathbf{p})=(2 \pi)^{3} \sum_{a= \pm} \delta\left(p_{\mathrm{z}}-a K_{\mathrm{z}}^{\prime}\left(\mathbf{p}_{\perp}\right)\right) \overline{\mathscr{C}}^{a a}\left(Z, \mathbf{p}_{\perp}\right)$,

where the transverse component $\overline{\mathscr{C}}^{a a}\left(Z, \mathbf{p}_{\perp}\right)$ is given by

$$
\begin{aligned}
\mathscr{\mathscr { C }}^{a a}\left(Z, \mathbf{p}_{\perp}\right)= & \frac{1}{(2 \pi)^{5}} \int_{0}^{H}\left[\int \overline{\overline{\mathcal{G}}}^{a a}\left(Z, \mathbf{p}_{\perp}, Z_{1}, \mathbf{p}_{1}, 0\right)\right. \\
& \left.\cdot \overline{\overline{\mathbf{I}}}_{\mathrm{WF}}\left(Z_{1}, \mathbf{p}_{1}, Z_{2}, \mathbf{p}_{2}, 0\right) \cdot \overline{\mathbf{C}}_{\mathrm{W}}\left(Z_{2}, \mathbf{p}_{2}\right) \mathrm{d}^{3} \mathbf{p}_{1} \mathrm{~d}^{3} \mathbf{p}_{2}\right] \mathrm{d} Z_{1} \mathrm{~d} Z_{2} .
\end{aligned}
$$

Hereafter, we give a physical interpretation of the Wigner transform of the diffuse dyadic correlation function, and in particular of its transverse component. We employ the same analysis as in Ref. $[10,11]$ with the aim to recover the representation (137). Let us decompose the diffuse field into a spectrum of up-going and downgoing waves

$$
\begin{aligned}
\mathscr{E}^{1}(\mathbf{r})= & \int\left[\mathscr{E}_{+}^{1}\left(z, \mathbf{k}_{\perp}\right) \mathrm{e}^{\mathrm{j} K_{z}\left(\mathbf{k}_{\perp}\right) z}+\mathscr{E}_{-}^{1}\left(z, \mathbf{k}_{\perp}\right) \mathrm{e}^{-\mathrm{j} K_{z}\left(\mathbf{k}_{\perp}\right) z}\right] \\
& \times \mathrm{e}^{\mathrm{j} \mathbf{k}_{\perp} \cdot \mathbf{r}_{\perp}} \mathrm{d}^{2} \mathbf{k}_{\perp},
\end{aligned}
$$

with

$\mathscr{E}_{a}^{1}\left(z, \mathbf{k}_{\perp}\right)=\mathscr{E}_{a \varphi}^{1}\left(z, \mathbf{k}_{\perp}\right) \widehat{\boldsymbol{\varphi}}\left(\widehat{\mathbf{k}}^{a}\right)+\mathscr{E}_{a \theta}^{1}\left(z, \mathbf{k}_{\perp}\right) \widehat{\boldsymbol{\theta}}\left(\widehat{\mathbf{k}}^{a}\right)$,

for $\mathbf{k}^{a}=\mathbf{k}_{\perp}+a K_{\mathbf{z}}^{\prime}\left(\mathbf{k}_{\perp}\right) \widehat{\mathbf{z}}$. In Eq. (139), $\mathscr{E}_{+}^{1}\left(z, \mathbf{k}_{\perp}\right)$ is the incoherent envelope amplitude of the up-going waves with transverse wave vector $\mathbf{k}_{\perp}$, while $\mathscr{E}_{-}^{1}\left(z, \mathbf{k}_{\perp}\right)$ is the corresponding incoherent envelope amplitude of the down-going waves. The $z$-dependence retained in $\mathscr{E}_{a}^{1}\left(z, \mathbf{k}_{\perp}\right)$ is the mean free path scale $l_{\text {mp }}\left(z \approx l_{\text {mp }}\right)$. The dyadic correlation functions for the envelope amplitudes are assumed to be of the form

$\left\langle\mathscr{E}_{a}^{1}\left(z, \mathbf{k}_{\perp}\right) \otimes \mathscr{E}_{b}^{1 \star}\left(z^{\prime}, \mathbf{k}_{\perp}^{\prime}\right)\right\rangle_{V}=\delta\left(\mathbf{k}_{\perp}-\mathbf{k}_{\perp}^{\prime}\right) \overline{\boldsymbol{\Lambda}}_{a b}\left(Z, \mathbf{k}_{\perp}\right), \quad a, b= \pm$,

where as usual, $Z=\left(z+z^{\prime}\right) / 2$. This means that (i) $\overline{\boldsymbol{\Lambda}}_{a b}$ varies only with respect to $Z$ on the mean free path scale $l_{\text {mp }}$ and not with respect to $z-z^{\prime}$, which is on the wavelength scale $\lambda_{e}$; (ii) the fields with the same $\mathbf{k}_{\perp}$ are correlated, while the fields with different $\mathbf{k}_{\perp}$ are uncorrelated; and (iii) the correlations due to the reflecting boundaries of the up-going and down-going waves are represented by $\overline{\boldsymbol{\Lambda}}_{a-a}$. From Eqs. (139) and (141), we find the following expression for the diffuse dyadic correlation function:

$\overline{\mathscr{C}}\left(\mathbf{r}, \mathbf{r}^{\prime}\right)=\int \bar{\Lambda}\left(Z, \mathbf{k}_{\perp}\right) \mathrm{e}^{\mathrm{j}_{\perp} \cdot\left(\mathbf{r}_{\perp}-\mathbf{r}_{\perp}^{\prime}\right)} \mathrm{d}^{2} \mathbf{k}_{\perp}$,

where

$$
\begin{aligned}
\overline{\boldsymbol{\Lambda}}\left(Z, \mathbf{k}_{\perp}\right)= & \sum_{a= \pm} \overline{\boldsymbol{\Lambda}}_{a}\left(Z, \mathbf{k}_{\perp}\right) \mathrm{e}^{\mathrm{j} a\left[K_{\mathbf{z}}\left(\mathbf{k}_{\perp}\right) z-K_{\mathrm{z}}^{\star}\left(\mathbf{k}_{\perp}\right) z^{\prime}\right]} . \\
& +\sum_{a= \pm} \overline{\boldsymbol{\Lambda}}_{a-a}\left(Z, \mathbf{k}_{\perp}\right) \mathrm{e}^{\mathrm{j} a\left[K_{\mathbf{z}}\left(\mathbf{k}_{\perp}\right) z+K_{z}^{\star}\left(\mathbf{k}_{\perp}\right) z^{\prime}\right]} .
\end{aligned}
$$

Further on, neglecting the correlations due to the reflecting boundaries, i.e., neglecting the dyadics $\overline{\boldsymbol{\Lambda}}_{a-a}\left(Z, \mathbf{k}_{\perp}\right)$ in Eq. (143), we get

$$
\begin{aligned}
\overline{\mathscr{C}}\left(\mathbf{r}, \mathbf{r}^{\prime}\right)= & \sum_{a= \pm} \int \overline{\boldsymbol{\Lambda}}_{a a}\left(Z, \mathbf{k}_{\perp}\right) \mathrm{e}^{-2 a K_{z}^{\prime \prime}\left(\mathbf{k}_{\perp}\right) Z} \\
& \times \mathrm{e}^{\mathrm{j} a K_{z}^{\prime}\left(\mathbf{k}_{\perp}\right)\left(z-z^{\prime}\right)} \mathrm{e}^{\mathrm{j} \mathbf{k}_{\perp} \cdot\left(\mathbf{r}_{\perp}-\mathbf{r}_{\perp}^{\prime}\right)} \mathrm{d}^{2} \mathbf{k}_{\perp} .
\end{aligned}
$$

For $\left\langle\overline{\mathscr{C}}\left(\mathbf{r}, \mathbf{r}^{\prime}\right)\right\rangle_{S}$ we assume a similar representation, with say, $\overline{\boldsymbol{\Lambda}}_{a a}\left(Z, \mathbf{k}_{\perp}\right)$ in place of $\overline{\boldsymbol{\Lambda}}_{a a}\left(Z, \mathbf{k}_{\perp}\right)$. Then, by straightforward calculations, we find that the Wigner transform of $\left\langle\overline{\mathscr{C}}\left(\mathbf{r}, \mathbf{r}^{\prime}\right)\right\rangle_{S}$ is of the form

$\overline{\mathscr{C}}_{\mathrm{W}}(\mathbf{R}, \mathbf{p})=\overline{\mathscr{C}}_{\mathrm{W}}(Z, \mathbf{p})$,

with

$\overline{\mathscr{C}}_{\mathrm{W}}(Z, \mathbf{p})=(2 \pi)^{3} \sum_{a= \pm} \delta\left(p_{\mathrm{z}}-a K_{\mathrm{z}}^{\prime}\left(\mathbf{p}_{\perp}\right)\right) \overline{\mathscr{C}}^{a a}\left(Z, \mathbf{p}_{\perp}\right)$,

$\overline{\mathscr{C}}^{a a}\left(Z, \mathbf{p}_{\perp}\right)=\mathrm{e}^{-2 a K_{z}^{\prime \prime}\left(\mathbf{p}_{\perp}\right) Z} \overline{\boldsymbol{\Lambda}}_{a a}\left(Z, \mathbf{p}_{\perp}\right)$.

Thus, both representations for $\overline{\mathscr{C}}_{\mathrm{W}}(Z, \mathbf{p})$, i.e., (137) and (146), are identical. Except for the transmission factor $\exp \left[-2 a K_{\mathrm{z}}^{\prime \prime}\left(\mathbf{p}_{\perp}\right) Z\right]$, the transverse $\left(\mathbf{p}_{\perp-}\right)$ components of the Wigner transform $\mathscr{C}_{\mathrm{W}}(Z, \mathbf{p})$, $\overline{\mathscr{C}}^{a a}\left(Z, \mathbf{p}_{\perp}\right)$ coincide with the dyadic correlation functions for the (incoherent) envelope amplitudes $\overline{\boldsymbol{\Lambda}}_{a a}\left(Z, \mathbf{p}_{\perp}\right)$. Some comments can be made here.

1. Eq. (145) shows that the problem has translational invariance in azimuth.

2. Owing to Eqs. (140), (141), and (147), $\overline{\mathscr{C}}^{a a}\left(Z, \mathbf{p}_{\perp}\right)$ is a transverse dyadic, i.e.,

$\overline{\mathscr{C}}^{a a}\left(Z, \mathbf{p}_{\perp}\right)=\sum_{\eta, \mu=\varphi, \theta}\left[\overline{\mathscr{C}}^{a a}\left(Z, \mathbf{p}_{\perp}\right)\right]_{\eta \mu} \widehat{\boldsymbol{\eta}}\left(\widehat{\mathbf{p}}^{a}\right) \otimes \widehat{\boldsymbol{\mu}}\left(\widehat{\mathbf{p}}^{a}\right)$.

3. The representations (123) and (146) of the Wigner transforms $\overline{\mathbf{C}}_{\mathrm{cW}}(Z, \mathbf{p})$ and $\overline{\mathscr{C}}_{\mathrm{W}}(Z, \mathbf{p})$, respectively, are of the same type. Therefore, the Wigner transform of the (total) dyadic correlation function inherits this representation type; it is given by

$$
\begin{aligned}
\overline{\mathbf{C}}_{\mathrm{W}}(Z, \mathbf{p}) & =\overline{\mathbf{C}}_{\mathrm{cW}}(Z, \mathbf{p})+\overline{\mathscr{C}}_{\mathrm{W}}(Z, \mathbf{p}) \\
& =(2 \pi)^{3} \sum_{a= \pm} \delta\left(p_{\mathrm{z}}-a K_{\mathrm{z}}^{\prime}\left(\mathbf{p}_{\perp}\right)\right) \overline{\mathbf{C}}^{a a}\left(Z, \mathbf{p}_{\perp}\right),
\end{aligned}
$$

where

$\overline{\mathbf{C}}^{a a}\left(Z, \mathbf{p}_{\perp}\right)=\overline{\mathbf{C}}_{\mathrm{c}}^{a a}\left(Z, \mathbf{p}_{\perp}\right)+\overline{\mathscr{C}}^{a a}\left(Z, \mathbf{p}_{\perp}\right)$. 
In summary, when all cross terms in the expression of the tetradic Green's function $\overline{\overline{\mathbf{G}}}\left(\mathbf{r}, \mathbf{r}_{1}, \mathbf{r}^{\prime}, \mathbf{r}_{1}^{\prime}\right)$ are excluded, the integral equation (103), derived under the effective field approximation, implies that the Wigner transform $\overline{\mathscr{C}}_{\mathrm{W}}(Z, \mathbf{p})$

1. includes only the uncrossed dyadics $\overline{\mathscr{C}}^{a a}\left(Z, \mathbf{p}_{\perp}\right)$, and

2. depends on $p_{z}$ through the Dirac delta function $\delta\left(p_{z}-\right.$ $\left.a K_{\mathrm{z}}^{\prime}\left(\mathbf{p}_{\perp}\right)\right)$. lows

In the above framework, these results can be explained as fol-

1. If in Eq. (143) we disregard the dyadic correlation functions for the envelope amplitudes $\overline{\boldsymbol{\Lambda}}_{a-a}\left(z, z^{\prime}, \mathbf{k}_{\perp}\right)$ then $\overline{\mathscr{C}}_{\mathrm{W}}(Z, \mathbf{p})$ will include only the uncrossed dyadics $\overline{\mathscr{C}}^{a a}\left(Z, \mathbf{p}_{\perp}\right)$; the cross dyadics $\overline{\mathscr{C}}^{a-a}\left(Z, \mathbf{p}_{\perp}\right)$ corresponding to $\overline{\boldsymbol{\Lambda}}_{a-a}\left(z, z^{\prime}, \mathbf{k}_{\perp}\right)$ are excluded.

2. By virtue of the assumption (141), which is similar to the quasiuniform field approximation, $\overline{\mathscr{C}}_{\mathrm{W}}(Z, \mathbf{p})$ depends on $p_{\mathrm{z}}$ through the Dirac delta function $\delta\left(p_{z}-a K_{z}^{\prime}\left(\mathbf{p}_{\perp}\right)\right)$. In fact, the representation (149) of the Wigner transform of the dyadic correlation function is equivalent to the representation (2) in the quasiuniform field approximation.

\subsection{Radiative transfer equation}

The radiative transfer equation will be formulated for $\overline{\mathscr{C}}^{a a}\left(Z, \mathbf{p}_{\perp}\right)$, which are the upward and downward transverse components of the Wigner transform $\overline{\mathscr{C}}_{\mathrm{W}}(Z, \mathbf{p})$.

2.6.1. Integral and differential forms of the radiative transfer equation Inserting Eqs. (106), (107), (110), and (118) on one hand and Eqs. (123) and (146) on the other hand in Eq. (103), and tracing up-going and down-going waves, we obtain the following integral form of the radiative transfer equation:

$$
\begin{aligned}
\overline{\mathscr{C}}^{a a}\left(Z, \mathbf{p}_{\perp}\right) \\
=\frac{n_{0}}{16 \pi^{2}} \mathrm{e}^{-2 a K_{z}^{\prime \prime}\left(\mathbf{p}_{\perp}\right) Z} \sum_{b, c= \pm} \int_{0}^{H}\left\{\int \frac{1}{\left|K_{z}\left(\mathbf{p}_{1 \perp}\right)\right|^{2}} \mathrm{e}^{2 b K_{z}^{\prime \prime}\left(\mathbf{p}_{\perp \perp}\right) Z_{1}}\right. \\
\quad \times\left[\overline{\overline{\mathcal{S}}}^{1 a 1 b}\left(\mathbf{p}_{\perp}, \mathbf{p}_{1 \perp}, 0\right)+\delta_{a b} \delta_{a, \operatorname{sgn}\left(Z-Z_{1}\right)} \delta\left(\mathbf{p}_{\perp}-\mathbf{p}_{1 \perp}\right) \overline{\overline{\mathbf{I}}}_{\perp}^{a b}\left(\mathbf{p}_{\perp}\right)\right] \\
\left.\quad \cdot \overline{\overline{\mathbf{T}}}_{p \mathrm{~T}}^{b c}\left(\mathbf{p}_{1 \perp}, \mathbf{p}_{2 \perp}\right) \cdot\left[\overline{\mathbf{C}}_{c}^{c c}\left(Z_{1}, \mathbf{p}_{2 \perp}\right)+\overline{\mathscr{C}}^{c c}\left(Z_{1}, \mathbf{p}_{2 \perp}\right)\right] \mathrm{d}^{2} \mathbf{p}_{1 \perp} \mathrm{d}^{2} \mathbf{p}_{2 \perp}\right\} \mathrm{d} Z_{1},
\end{aligned}
$$

where

$\overline{\overline{\mathbf{T}}}_{p \mathrm{~T}}^{b c}\left(\mathbf{p}_{1 \perp}, \mathbf{p}_{2 \perp}\right)=\overline{\mathbf{T}}_{p \mathrm{~T}}\left(\mathbf{p}_{1}^{b}, \mathbf{p}_{2}^{c}\right) \otimes \overline{\mathbf{T}}_{p \mathrm{~T}}^{\star}\left(\mathbf{p}_{1}^{b}, \mathbf{p}_{2}^{c}\right)$.

Some comments in connection with the derivation of the integral equation (151) are in order.

1. In Eq. (103), the Dirac delta functions $\delta\left(p_{1 z}-b K_{z}^{\prime}\left(\mathbf{p}_{1 \perp}\right)\right)$ and $\delta\left(p_{2 \mathrm{z}}-c K_{\mathrm{z}}^{\prime}\left(\mathbf{p}_{2 \perp}\right)\right)$ appearing in the expressions for $\overline{\overline{\mathbf{G}}}_{\mathrm{WF}}\left(\cdot, \mathbf{p}_{1}, \cdot\right)$ and $\overline{\mathbf{C}}_{\mathrm{W}}\left(\cdot, \mathbf{p}_{2}\right)$, respectively, yield $\operatorname{sgn}\left(p_{1 \mathrm{z}}\right)=b$ and $\operatorname{sgn}\left(p_{2 \mathrm{z}}\right)=c$; therefore, in Eq. (118), we have $\mathbf{p}_{1}^{\operatorname{sgn}\left(p_{1 z}\right)}=\mathbf{p}_{1}^{b}$ and $\mathbf{p}_{2}^{\operatorname{sgn}\left(p_{2 z}\right)}=\mathbf{p}_{2}^{c}$, and so,

$$
\begin{aligned}
& \overline{\mathbf{T}}_{p}\left(\mathbf{p}_{1}^{\operatorname{sgn}\left(p_{1 z}\right)}, \mathbf{p}_{2}^{\operatorname{sgn}\left(p_{2 z}\right)}\right) \otimes \overline{\mathbf{T}}_{p}^{\star}\left(\mathbf{p}_{1}^{\operatorname{sgn}\left(p_{1 z}\right)}, \mathbf{p}_{2}^{\operatorname{sgn}\left(p_{2 z}\right)}\right) \\
& \quad=\overline{\mathbf{T}}_{p}\left(\mathbf{p}_{1}^{b}, \mathbf{p}_{2}^{c}\right) \otimes \overline{\mathbf{T}}_{p}^{\star}\left(\mathbf{p}_{1}^{b}, \mathbf{p}_{2}^{c}\right) .
\end{aligned}
$$

2. If $\overline{\mathbf{X}}$ is the dyadic $\overline{\mathbf{X}}=\sum_{i j} X_{i j} \widehat{\mathbf{e}}_{i} \otimes \widehat{\mathbf{e}}_{j}$, then the computation rule

$$
\left[\left(\widehat{\mathbf{e}}_{i} \otimes \widehat{\mathbf{e}}_{j}\right) \otimes\left(\widehat{\mathbf{e}}_{k} \otimes \widehat{\mathbf{e}}_{l}\right)\right] \cdot\left(\widehat{\mathbf{e}}_{j^{\prime}} \otimes \widehat{\mathbf{e}}_{l^{\prime}}\right)=\left(\widehat{\mathbf{e}}_{j} \cdot \widehat{\mathbf{e}}_{j^{\prime}}\right)\left(\widehat{\mathbf{e}}_{l} \cdot \widehat{\mathbf{e}}_{l^{\prime}}\right) \widehat{\mathbf{e}}_{i} \otimes \widehat{\mathbf{e}}_{k},
$$

$$
\begin{aligned}
& \text { gives (cf. Eq. (111)) } \\
& \overline{\overline{\mathcal{S}}}^{1 a 1 b}\left(\mathbf{p}_{\perp}, \mathbf{p}_{1 \perp}, 0\right) \cdot \overline{\mathbf{X}}=\sum_{\eta, \mu, \eta^{\prime}, \mu^{\prime}=\varphi, \theta} \sum_{i j}\left[\overline{\mathcal{\mathcal { S }}}^{1 a 1 b}\left(\mathbf{p}_{\perp}, \mathbf{p}_{1 \perp}, 0\right)\right]_{\eta \mu \eta^{\prime} \mu^{\prime}} \\
& \quad \times X_{i j}\left[\widehat{\boldsymbol{\mu}}\left(\widehat{\mathbf{p}}_{1}^{b}\right) \cdot \widehat{\mathbf{e}}_{i}\right]\left[\widehat{\boldsymbol{\mu}}^{\prime}\left(\widehat{\mathbf{p}}_{1}^{b}\right) \cdot \widehat{\mathbf{e}}_{j}\right] \widehat{\boldsymbol{\eta}}\left(\widehat{\mathbf{p}}^{a}\right) \otimes \widehat{\boldsymbol{\eta}}^{\prime}\left(\widehat{\mathbf{p}}^{a}\right) .
\end{aligned}
$$

Thus, $\overline{\overline{\mathcal{S}}}^{1 a 1 b}\left(\mathbf{p}_{\perp}, \mathbf{p}_{1 \perp}, 0\right) \cdot \overline{\mathbf{X}}$ is a transverse dyadic, and the same result is valid for the dyadic $\overline{\mathbf{I}}_{\perp}^{a b}\left(\mathbf{p}_{\perp}\right) \cdot \overline{\mathbf{X}}$. Consequently, from the integral equation (151), we deduce that $\overline{\mathscr{C}}^{a a}\left(Z, \mathbf{p}_{\perp}\right)$ is also a transverse dyadic as in Eq. (148). Thus, the transversality of $\overline{\mathscr{C}}^{a a}\left(Z, \mathbf{p}_{\perp}\right)$ may follow directly from the integral Eq. (151).

3. Using the computation rule (154), and taking into account that $\overline{\overline{\mathcal{S}}}^{1 a 1 b}\left(\mathbf{p}_{\perp}, \mathbf{p}_{1 \perp}, 0\right)$ and $\overline{\overline{\mathbf{I}}}_{\perp}^{a b}\left(\mathbf{p}_{\perp}\right)$ are transverse tetradics, and that $\overline{\mathbf{C}}_{\mathrm{c}}^{c C}\left(Z_{1}, \mathbf{p}_{2 \perp}\right)$ and $\overline{\mathscr{C}}^{c c}\left(Z_{1}, \mathbf{p}_{2 \perp}\right)$ are transverse dyadics, we find, for example, that

$$
\begin{aligned}
\overline{\overline{\mathcal{S}}}^{1 a 1 b}\left(\mathbf{p}_{\perp}, \mathbf{p}_{1 \perp}, 0\right) \cdot\left[\overline{\mathbf{T}}_{p}\left(\mathbf{p}_{1}^{b}, \mathbf{p}_{2}^{c}\right) \otimes \overline{\mathbf{T}}_{p}^{\star}\left(\mathbf{p}_{1}^{b}, \mathbf{p}_{2}^{c}\right)\right] \cdot \overline{\mathbf{C}}_{\mathrm{c}}^{c c}\left(Z_{1}, \mathbf{p}_{2 \perp}\right) \\
=\left[\overline{\overline{\mathcal{S}}}^{1 a 1 b}\left(\mathbf{p}_{\perp}, \mathbf{p}_{1 \perp}, 0\right) \cdot \overline{\overline{\mathbf{I}}}_{\perp}^{b b}\left(\mathbf{p}_{1 \perp}\right)\right] \\
\cdot\left[\overline{\mathbf{T}}_{p}\left(\mathbf{p}_{1}^{b}, \mathbf{p}_{2}^{c}\right) \otimes \overline{\mathbf{T}}_{p}^{\star}\left(\mathbf{p}_{1}^{b}, \mathbf{p}_{2}^{c}\right)\right] \cdot\left[\overline{\overline{\mathbf{I}}}_{\perp}^{c c}\left(\mathbf{p}_{2 \perp}\right) \cdot \overline{\mathbf{C}}_{\mathrm{c}}^{c c}\left(Z_{1}, \mathbf{p}_{2 \perp}\right)\right] \\
=\overline{\overline{\mathcal{S}}}^{1 a 1 b}\left(\mathbf{p}_{\perp}, \mathbf{p}_{1 \perp}, 0\right) \cdot \overline{\overline{\mathbf{T}}}_{p \mathrm{~T}}^{b c}\left(\mathbf{p}_{1 \perp}, \mathbf{p}_{2 \perp}\right) \cdot \overline{\mathbf{C}}_{\mathrm{c}}^{c c}\left(Z_{1}, \mathbf{p}_{2 \perp}\right),
\end{aligned}
$$

where, be means of the dyadic identity

$(\overline{\mathbf{A}} \otimes \overline{\mathbf{B}}) \cdot(\overline{\mathbf{C}} \otimes \overline{\mathbf{D}})=(\overline{\mathbf{A}} \cdot \overline{\mathbf{C}}) \otimes(\overline{\mathbf{B}} \cdot \overline{\mathbf{D}})$,

$\overline{\overline{\mathbf{T}}}_{p \mathrm{~T}}^{b c}\left(\mathbf{p}_{1 \perp}, \mathbf{p}_{2 \perp}\right)$ is given by (cf. Eq. (152))

$$
\begin{aligned}
\overline{\overline{\mathbf{T}}}_{p \mathrm{~T}}^{b c}\left(\mathbf{p}_{1 \perp}, \mathbf{p}_{2 \perp}\right) & =\overline{\overline{\mathbf{I}}}_{\perp}^{b b}\left(\mathbf{p}_{1 \perp}\right) \cdot\left[\overline{\mathbf{T}}_{p}\left(\mathbf{p}_{1}^{b}, \mathbf{p}_{2}^{c}\right) \otimes \overline{\mathbf{T}}_{p}^{\star}\left(\mathbf{p}_{1}^{b}, \mathbf{p}_{2}^{c}\right)\right] \cdot \overline{\overline{\mathbf{I}}}_{\perp}^{c c}\left(\mathbf{p}_{2 \perp}\right) \\
& =\left[\overline{\mathbf{I}}_{\perp}^{b}\left(\mathbf{p}_{1 \perp}\right) \cdot \overline{\mathbf{T}}_{p}\left(\mathbf{p}_{1}^{b}, \mathbf{p}_{2}^{c}\right) \cdot \overline{\mathbf{I}}_{\perp}^{c}\left(\mathbf{p}_{2 \perp}\right)\right] \\
& \otimes\left[\overline{\mathbf{I}}_{\perp}^{b}\left(\mathbf{p}_{1 \perp}\right) \cdot \overline{\mathbf{T}}_{p}^{\star}\left(\mathbf{p}_{1}^{b}, \mathbf{p}_{2}^{c}\right) \cdot \overline{\mathbf{I}}_{\perp}^{c}\left(\mathbf{p}_{2 \perp}\right)\right] \\
& =\overline{\mathbf{T}}_{p \mathrm{~T}}\left(\mathbf{p}_{1}^{b}, \mathbf{p}_{2}^{c}\right) \otimes \overline{\mathbf{T}}_{p \mathrm{~T}}^{\star}\left(\mathbf{p}_{1}^{b}, \mathbf{p}_{2}^{c}\right)
\end{aligned}
$$

Taking the derivative of Eq. (151) and using

$$
\begin{aligned}
\delta_{a, \operatorname{sgn}\left(Z-Z_{1}\right)} & =\delta_{a+} H\left(Z-Z_{1}\right)+\delta_{a-} H\left(Z_{1}-Z\right), \\
\frac{\mathrm{d} H(Z)}{\mathrm{d} Z} & =\delta(Z),
\end{aligned}
$$

which gives

$$
\begin{aligned}
& \frac{\mathrm{d}}{\mathrm{d} Z} \int_{0}^{H} f\left(Z_{1}\right) \delta_{a, \operatorname{sgn}\left(Z-Z_{1}\right)} \mathrm{d} Z_{1} \\
& =\int_{0}^{H} f\left(Z_{1}\right)\left[\delta_{a+} \delta\left(Z-Z_{1}\right)-\delta_{a-} \delta\left(Z_{1}-Z\right)\right] \mathrm{d} Z_{1} \\
& =a f(Z),
\end{aligned}
$$

where $H(Z)$ is the Heaviside step-function, we obtain the differential form of the radiative transfer equation:

$$
\begin{aligned}
& \frac{\partial}{\partial Z} \overline{\mathscr{C}}^{a a}\left(Z, \mathbf{p}_{\perp}\right) \\
& =-2 a K_{\mathrm{z}}^{\prime \prime}\left(\mathbf{p}_{\perp}\right) \overline{\mathscr{C}}^{a a}\left(Z, \mathbf{p}_{\perp}\right)+\frac{a}{16 \pi^{2}} \frac{n_{0}}{\left|K_{\mathrm{z}}\left(\mathbf{p}_{\perp}\right)\right|^{2}} \\
& \quad \times \sum_{b= \pm} \int \overline{\mathbf{T}}_{p \mathrm{~T}}^{a b}\left(\mathbf{p}_{\perp}, \mathbf{p}_{1 \perp}\right) \cdot\left[\overline{\mathbf{C}}_{\mathrm{c}}^{b b}\left(Z, \mathbf{p}_{1 \perp}\right)+\overline{\mathscr{C}}^{b b}\left(Z, \mathbf{p}_{1 \perp}\right)\right] \mathrm{d}^{2} \mathbf{p}_{1 \perp} .
\end{aligned}
$$

Note that in deriving Eq. (159), we used the result

$\overline{\overline{\mathbf{I}}}_{\perp}^{a a}\left(\mathbf{p}_{\perp}\right) \cdot \overline{\overline{\mathbf{T}}}_{p \mathrm{~T}}^{a b}\left(\mathbf{p}_{\perp}, \mathbf{p}_{1 \perp}\right)=\overline{\overline{\mathbf{T}}}_{p \mathrm{~T}}^{a b}\left(\mathbf{p}_{\perp}, \mathbf{p}_{1 \perp}\right)$,

which follows from the dyadic identity (157). The differential Eq. (159) is complemented with the boundary conditions

$\overline{\mathscr{C}}^{++}\left(0, \mathbf{p}_{\perp}\right)=\int \overline{\overline{\mathcal{R}}}_{01}\left(\mathbf{p}_{\perp}, \mathbf{p}_{1 \perp}, 0\right) \cdot \overline{\mathscr{C}}^{--}\left(0, \mathbf{p}_{1 \perp}\right) \mathrm{d}^{2} \mathbf{p}_{1 \perp}$ 
at $Z=0$, and

$\overline{\mathscr{C}}^{--}\left(H, \mathbf{p}_{\perp}\right)=\int \overline{\overline{\mathcal{R}}}_{21}\left(\mathbf{p}_{\perp}, \mathbf{p}_{1 \perp}, 0\right) \cdot \overline{\mathscr{C}}^{++}\left(H, \mathbf{p}_{1 \perp}\right) \mathrm{d}^{2} \mathbf{p}_{1 \perp}$

at $Z=H$. Here, the tetradic reflection operator $\overline{\overline{\mathcal{R}}}_{01}\left(\mathbf{p}_{\perp}, \mathbf{p}_{1 \perp}, 0\right)$ at the lower boundary is defined by

$\overline{\overline{\mathcal{R}}}_{01}\left(\mathbf{p}_{\perp}, \mathbf{p}_{1 \perp}, 0\right)=\frac{(2 \pi)^{2}}{A}\left\langle\overline{\mathbf{R}}_{01}\left(\mathbf{p}_{\perp}, \mathbf{p}_{1 \perp}\right) \otimes \overline{\mathbf{R}}_{01}^{\star}\left(\mathbf{p}_{\perp}, \mathbf{p}_{1 \perp}\right)\right\rangle_{S}$,

while the tetradic reflection operator $\overline{\mathcal{R}}_{21}\left(\mathbf{p}_{\perp}, \mathbf{p}_{1 \perp}, \mathbf{P}_{\perp}\right)$ at the upper boundary is defined as in Eq. (135). The boundary conditions (160) and (161) are derived under the so-called weak surface correlation approximation discussed in Appendix 1.

\subsubsection{Conventional radiative transfer equation}

The conventional radiative transfer equation will be formulated for the transverse components $\overline{\mathbf{C}}^{a a}\left(Z, \mathbf{p}_{\perp}\right)$ of the Wigner transform of the (total) dyadic correlation function $\overline{\mathbf{C}}_{\mathrm{W}}(Z, \mathbf{p})$. By making use of Eqs. (131) and (150), we express the radiative transfer Eq. (159) in terms of $\overline{\mathbf{C}}^{a a}\left(Z, \mathbf{p}_{\perp}\right)$ as

$$
\begin{aligned}
\frac{\partial}{\partial Z} \overline{\mathbf{C}}^{a a}\left(Z, \mathbf{p}_{\perp}\right)= & -2 a K_{\mathrm{z}}^{\prime \prime}\left(\mathbf{p}_{\perp}\right) \overline{\mathbf{C}}^{a a}\left(Z, \mathbf{p}_{\perp}\right)+\frac{a}{16 \pi^{2}} \frac{n_{0}}{\left|K_{\mathrm{z}}\left(\mathbf{p}_{\perp}\right)\right|^{2}} \\
& \times \sum_{b= \pm} \int \overline{\mathbf{T}}_{p \mathrm{~T}}^{a b}\left(\mathbf{p}_{\perp}, \mathbf{p}_{1 \perp}\right) \cdot \overline{\mathbf{C}}^{b b}\left(Z, \mathbf{p}_{1 \perp}\right) \mathrm{d}^{2} \mathbf{p}_{1 \perp} .
\end{aligned}
$$

Then we proceed as follows.

1. We assume that $K^{\prime \prime} \ll K^{\prime}$, yielding $K \approx K^{\prime}$, and neglect the evanescent waves, i.e., $\left|\mathbf{p}_{\perp}\right|=p_{\perp} \leq K^{\prime}$. Recall that the disregard of the evanescent waves is equivalent to the far-field approximation for the fields. In this context, we define the real upward $(a=+)$ and downward $(a=-)$ vectors for propagating waves by

$\mathbf{p}^{a}=\mathbf{p}_{\perp}+a K_{\mathbf{z}}^{\prime}\left(\mathbf{p}_{\perp}\right) \widehat{\mathbf{z}}$

$$
K_{\mathrm{z}}^{\prime}\left(\mathbf{p}_{\perp}\right)=\sqrt{K^{\prime 2}-p_{\perp}^{2}} .
$$

Obviously, $\mathbf{p}^{a}$ is a real vector whose norm is exactly $K^{\prime}$, i.e., $\left|\mathbf{p}^{a}\right|=K^{\prime}$. Also note that only under the assumption $K^{\prime \prime} \ll K^{\prime}$, the new definition $K_{\mathrm{z}}^{\prime}\left(\mathbf{p}_{\perp}\right)=\sqrt{K^{\prime 2}-p_{\perp}^{2}}$ agrees with the previous one $K_{\mathrm{z}}^{\prime}\left(\mathbf{p}_{\perp}\right)=\operatorname{Re}\left(\sqrt{K^{2}-p_{\perp}^{2}}\right)$. Putting

$K^{\prime} \widehat{\mathbf{p}}^{a}=\mathbf{p}_{\perp}+a K_{\mathrm{z}}^{\prime}\left(\mathbf{p}_{\perp}\right) \widehat{\mathbf{z}}$

we describe up-going and down-going propagating waves through the (normalized) direction $\widehat{\mathbf{p}}=\widehat{\mathbf{p}}(\theta, \varphi)$, with $\widehat{\mathbf{p}}=\widehat{p}_{\mathrm{x}} \widehat{\mathbf{x}}+$ $\widehat{p}_{\mathrm{y}} \widehat{\mathbf{y}}+\widehat{p}_{\mathrm{z}} \widehat{\mathbf{z}}$ and

$\widehat{p}_{\mathrm{x}}=\sin \theta \cos \varphi, \widehat{p}_{\mathrm{y}}=\sin \theta \sin \varphi, \widehat{p}_{\mathrm{z}}=\cos \theta$,

as follows:

$\widehat{\mathbf{p}}^{+}=\widehat{\mathbf{p}}(\theta, \varphi), \quad \theta \in[0, \pi / 2), \varphi \in[0,2 \pi]$,

$\widehat{\mathbf{p}}^{-}=\widehat{\mathbf{p}}(\theta, \varphi), \quad \theta \in(\pi / 2, \pi], \varphi \in[0,2 \pi]$.

Thus, the polar angle $\theta$ specifies if a wave is up-going or downgoing. Noting that $\mathbf{p}_{\perp}=K^{\prime}\left(\widehat{p}_{\mathrm{x}} \widehat{\mathbf{x}}+\widehat{p}_{\mathrm{y}} \widehat{\mathbf{y}}\right)$, we find

$\mathrm{d}^{2} \mathbf{p}_{\perp}=K^{\prime 2} \sin \theta|\cos \theta| \mathrm{d} \theta \mathrm{d} \varphi$,

$K_{\mathrm{z}}^{\prime}\left(\mathbf{p}_{\perp}\right)=K^{\prime} \widehat{p}_{\mathrm{z}}=K^{\prime}|\cos \theta|$,

and so,

$\frac{\mathrm{d}^{2} \mathbf{p}_{\perp}}{K^{\prime} K_{\mathrm{z}}^{\prime}\left(\mathbf{p}_{\perp}\right)}=\sin \theta \mathrm{d} \theta \mathrm{d} \varphi=\mathrm{d}^{2} \widehat{\mathbf{p}}$
2. For small $K^{\prime \prime}$, we use the first-order Taylor expansion

$K_{\mathrm{z}}\left(\mathbf{p}_{\perp}\right)=K_{\mathrm{z}}^{\prime}\left(\mathbf{p}_{\perp}\right)+\mathrm{j} \frac{K^{\prime} K^{\prime \prime}}{K_{\mathrm{z}}^{\prime}\left(\mathbf{p}_{\perp}\right)}$,

to approximate

$K_{\mathrm{z}}^{\prime \prime}\left(\mathbf{p}_{\perp}\right)=\frac{1}{2 \mathrm{j}}\left[K_{\mathrm{z}}\left(\mathbf{p}_{\perp}\right)-K_{\mathrm{z}}^{\star}\left(\mathbf{p}_{\perp}\right)\right] \approx \frac{K^{\prime} K^{\prime \prime}}{K_{\mathrm{z}}^{\prime}\left(\mathbf{p}_{\perp}\right)}=\frac{K^{\prime \prime}}{|\cos \theta|}$.

3. We introduce the specific dyadic correlation function $\bar{\Sigma}\left(Z, \widehat{\mathbf{p}}^{a}\right)$ through the relation

$$
\overline{\mathbf{\Sigma}}\left(Z, \widehat{\mathbf{p}}^{a}\right)=\overline{\mathbf{C}}^{a a}\left(Z, \mathbf{p}_{\perp}\right) K^{\prime} K_{\mathrm{z}}^{\prime}\left(\mathbf{p}_{\perp}\right)=\overline{\mathbf{C}}^{a a}\left(Z, \mathbf{p}_{\perp}\right) K^{\prime 2}|\cos \theta| .
$$

In using Eq. (149), the inverse Wigner transform gives

$$
\begin{aligned}
\overline{\mathbf{C}}\left(\mathbf{r}, \mathbf{r}^{\prime}\right) & =\frac{1}{(2 \pi)^{3}} \int \overline{\mathbf{C}}_{\mathrm{w}}\left(\frac{\mathbf{r}+\mathbf{r}^{\prime}}{2}, \mathbf{p}\right) \mathrm{e}^{\mathrm{j} \mathbf{p} \cdot\left(\mathbf{r}-\mathbf{r}^{\prime}\right)} \mathrm{d}^{3} \mathbf{p} \\
& =\sum_{a= \pm} \int \overline{\mathbf{C}}^{a a}\left(Z, \mathbf{p}_{\perp}\right) \mathrm{e}^{\mathrm{j} a K_{\mathrm{z}}^{\prime}\left(\mathbf{p}_{\perp}\right)\left(z-z^{\prime}\right)} \mathrm{e}^{\mathrm{j} \mathbf{p}_{\perp} \cdot\left(\mathbf{r}_{\perp}-\mathbf{r}_{\perp}^{\prime}\right)} \mathrm{d}^{2} \mathbf{p}_{\perp} .
\end{aligned}
$$

Substituting Eq. (174) in Eq. (175) and neglecting evanescent waves, that is, for $K_{\mathrm{z}}^{\prime}\left(\mathbf{p}_{\perp}\right)$ as in Eq. (165) and $\int \mathrm{d}^{2} \mathbf{p}_{\perp}=$ $\int_{p_{\perp} \leq K^{\prime}} \mathrm{d}^{2} \mathbf{p}_{\perp}$, we obtain

$$
\begin{aligned}
& \overline{\mathbf{C}}\left(\mathbf{r}, \mathbf{r}^{\prime}\right)=\sum_{a= \pm} \int_{\Omega_{a}} \overline{\boldsymbol{\Sigma}}\left(Z, \widehat{\mathbf{p}}^{a}\right) \mathrm{e}^{\mathrm{j} K^{\prime} \widehat{\mathbf{p}}^{a} \cdot\left(\mathbf{r}-\mathbf{r}^{\prime}\right)} \mathrm{d}^{2} \widehat{\mathbf{p}} \\
& =\int \overline{\boldsymbol{\Sigma}}(Z, \widehat{\mathbf{p}}) \mathrm{e}^{\mathrm{j} K / \widehat{\mathbf{p}} \cdot\left(\mathbf{r}-\mathbf{r}^{\prime}\right)} \mathrm{d}^{2} \widehat{\mathbf{p}},
\end{aligned}
$$

where $\Omega_{+}$and $\Omega_{-}$are the upper and the lower hemisphere.

The radiative transfer equation for the specific dyadic correlation function $\overline{\boldsymbol{\Sigma}}(Z, \widehat{\mathbf{p}})$, expressed in dyadic-product representation as

$\overline{\boldsymbol{\Sigma}}(Z, \widehat{\mathbf{p}})=\sum_{\eta, \mu=\varphi, \theta}[\overline{\boldsymbol{\Sigma}}(Z, \widehat{\mathbf{p}})]_{\eta \mu} \widehat{\boldsymbol{\eta}}(\widehat{\mathbf{p}}) \otimes \widehat{\boldsymbol{\mu}}(\widehat{\mathbf{p}})$,

with $\widehat{\mathbf{p}}=\widehat{\mathbf{p}}(\theta, \varphi)$, is then

$$
\begin{aligned}
\cos \theta \frac{\partial}{\partial Z} \overline{\mathbf{\Sigma}}(Z, \widehat{\mathbf{p}})= & -2 K^{\prime \prime} \overline{\boldsymbol{\Sigma}}(Z, \widehat{\mathbf{p}}) \\
& +n_{0} \int\left[\overline{\mathbf{A}}\left(\widehat{\mathbf{p}}, \widehat{\mathbf{p}}_{1}\right) \otimes \overline{\mathbf{A}}\left(\widehat{\mathbf{p}}, \widehat{\mathbf{p}}_{1}\right)\right] \cdot \overline{\mathbf{\Sigma}}\left(z, \widehat{\mathbf{p}}_{1}\right) \mathrm{d}^{2} \widehat{\mathbf{p}}_{1} .
\end{aligned}
$$

In obtaining Eq. (178), we used the relation between the transverse component of the Fourier transform of the transition operator $\overline{\mathbf{T}}_{p \mathrm{~T}}$ and the far-field scattering dyadic $\overline{\mathbf{A}}$, i.e., $\overline{\mathbf{T}}_{p \mathrm{~T}}\left(K^{\prime} \hat{\mathbf{p}}, K^{\prime} \widehat{\mathbf{p}}_{1}\right)=$ $4 \pi \overline{\mathbf{A}}\left(\widehat{\mathbf{p}}, \widehat{\mathbf{p}}_{1}\right)$, giving

$$
\begin{aligned}
\overline{\overline{\mathbf{T}}}_{p \mathrm{~T}}^{a b}\left(\mathbf{p}_{\perp}, \mathbf{p}_{1 \perp}\right) & =\overline{\mathbf{T}}_{p \mathrm{~T}}\left(K^{\prime} \widehat{\mathbf{p}}^{a}, K^{\prime} \widehat{\mathbf{p}}_{1}^{b}\right) \otimes \overline{\mathbf{T}}_{p \mathrm{~T}}^{\star}\left(K^{\prime} \widehat{\mathbf{p}}^{a}, K^{\prime} \widehat{\mathbf{p}}_{1}^{b}\right) \\
& =16 \pi^{2}\left[\overline{\mathbf{A}}\left(\widehat{\mathbf{p}}^{a}, \widehat{\mathbf{p}}_{1}^{b}\right) \otimes \overline{\mathbf{A}}^{\star}\left(\widehat{\mathbf{p}}^{a}, \widehat{\mathbf{p}}_{1}^{b}\right)\right] .
\end{aligned}
$$

As compared to Eq. (159), the radiative transfer Eq. (178) has been derived by further assuming that $K^{\prime \prime} \ll K^{\prime}$ and by neglecting the evanescent waves.

In the case $\mathbf{r}=\mathbf{r}^{\prime}$, the dyadic correlation function $\overline{\mathbf{C}}\left(\mathbf{r}, \mathbf{r}^{\prime}\right)$ is the coherency dyadic $\overline{\mathbf{C}}(\mathbf{r})$, and the specific dyadic correlation function $\overline{\boldsymbol{\Sigma}}(Z, \widehat{\mathbf{p}})$ is the specific coherency dyadic $\overline{\boldsymbol{\Sigma}}(z, \widehat{\mathbf{p}})$, so that (cf. Eq. (176) with $\mathbf{r}=\mathbf{r}^{\prime}$ )

$\overline{\mathbf{C}}(\mathbf{r})=\int \overline{\boldsymbol{\Sigma}}(z, \widehat{\mathbf{p}}) \mathrm{d}^{2} \widehat{\mathbf{p}}$.

Because, $Z=Z=z^{\prime}$, the radiative transfer equation for the specific coherency dyadic is as in Eq. (178), but with $Z$ replaced by $z$. Putting

$\overline{\mathbf{A}}\left(\widehat{\mathbf{p}}, \widehat{\mathbf{p}}_{1}\right)=\sum_{\eta, \mu=\varphi, \theta}\left[\mathrm{S}\left(\widehat{\mathbf{p}}, \widehat{\mathbf{p}}_{1}\right)\right]_{\eta \mu} \widehat{\boldsymbol{\eta}}(\widehat{\mathbf{p}}) \otimes \widehat{\boldsymbol{\mu}}\left(\widehat{\mathbf{p}}_{1}\right)$, 
where $S\left(\widehat{\mathbf{p}}, \widehat{\mathbf{p}}_{1}\right)$ is the amplitude matrix for the incident and scattering directions $\widehat{\mathbf{p}}_{1}$ and $\widehat{\mathbf{p}}$, respectively, and using the computation rule (154), we obtain the following radiative transfer equation for the specific coherency dyadic:

$$
\begin{aligned}
\cos \theta \frac{\partial}{\partial z}[\overline{\mathbf{\Sigma}}(z, \widehat{\mathbf{p}})]_{\eta \mu}= & -2 K^{\prime \prime}[\overline{\boldsymbol{\Sigma}}(z, \widehat{\mathbf{p}})]_{\eta \mu} \\
& +n_{0} \sum_{\eta^{\prime}, \mu^{\prime}=\varphi, \theta} \int\left[\mathrm{S}\left(\widehat{\mathbf{p}}, \widehat{\mathbf{p}}_{1}\right)\right]_{\eta \eta^{\prime}}\left[\mathrm{S}^{\star}\left(\widehat{\mathbf{p}}, \widehat{\mathbf{p}}_{1}\right)\right]_{\mu \mu^{\prime}} \\
& \times\left[\overline{\boldsymbol{\Sigma}}\left(z, \widehat{\mathbf{p}}_{1}\right)\right]_{\eta^{\prime} \mu^{\prime}} \mathrm{d}^{2} \widehat{\mathbf{p}}_{1} .
\end{aligned}
$$

To derive the radiative transfer equations for the specific coherency column vector, we first note that for spherical particles,

$K-K^{\star}=\frac{2 \pi}{k_{1}} n_{0}\left[S(0)-S^{\star}(0)\right]=\mathrm{j} n_{0} C_{\text {ext }}$,

where $S(0) \stackrel{\text { def }}{=}[\mathbf{S}(\widehat{\mathbf{s}}, \widehat{\mathbf{s}})]_{\theta \theta}=[\mathbf{S}(\widehat{\mathbf{s}}, \widehat{\mathbf{s}})]_{\varphi \varphi}, \mathbf{S}(\widehat{\mathbf{s}}, \widehat{\mathbf{s}})$ is the amplitude matrix in the forward direction, and $C_{\text {ext }}$ is the extinction cross section. Hence, $2 K^{\prime \prime}=n_{0} C_{\text {ext }}$. Let us define the multiindex $v=(\eta, \mu)$, such that $v$ takes the values $v=1,2,3,4$ for $(\eta, \mu)=(\theta, \theta),(\theta$, $\varphi),(\varphi, \theta),(\varphi, \varphi)$. Then, in terms of the specific coherency column vector

$[\mathrm{J}(z, \widehat{\mathbf{p}})]_{(\eta, \mu)}=\frac{1}{2} \sqrt{\frac{\varepsilon_{1}}{\mu_{0}}}[\bar{\Sigma}(z, \widehat{\mathbf{p}})]_{\eta \mu}$,

the radiative transfer Eq. (181) becomes

$$
\begin{aligned}
\cos \theta \frac{\mathrm{dJ}(z, \widehat{\mathbf{p}})}{\mathrm{d} z}= & -n_{0} \mathrm{~K}_{\mathrm{J}} \mathrm{J}(z, \widehat{\mathbf{p}}) \\
& +n_{0} \int \mathrm{z}_{\mathrm{J}}\left(\widehat{\mathbf{p}}, \widehat{\mathbf{p}}_{1}\right) \mathrm{J}\left(z, \widehat{\mathbf{p}}_{1}\right) \mathrm{d}^{2} \widehat{\mathbf{p}}_{1} .
\end{aligned}
$$

Here, the coherency extinction matrix is

$\mathrm{K}_{\mathrm{J}}=C_{\text {ext }} \mathrm{I}_{4}$,

where $\mathrm{I}_{4}$ is the four-by-four identity matrix, while the coherency phase matrix is

$\left[\mathrm{Z}_{\mathrm{J}}\left(\widehat{\mathbf{p}}, \widehat{\mathbf{p}}_{1}\right)\right]_{(\eta, \mu)\left(\eta^{\prime}, \mu^{\prime}\right)}=\left[\mathrm{S}\left(\widehat{\mathbf{p}}, \widehat{\mathbf{p}}_{1}\right)\right]_{\eta \eta^{\prime}}\left[\mathrm{S}^{\star}\left(\widehat{\mathbf{p}}, \widehat{\mathbf{p}}_{1}\right)\right]_{\mu \mu^{\prime}}$.

\section{Conclusions}

Following Ref. [16] as a template, we summarize below the assumptions under which the radiative transfer equation (159) has been derived.

A1. The fluctuations of the discrete random medium and the rough surfaces are assumed to be statistical independent and homogeneous. On average, the problem is translationally invariant and isotropic in azimuth. The fact that the problem has translational invariance in azimuth is reflected by the properties (96) and (97). By direct calculations, it has been shown that these properties are satisfied.

A2. By means of the effective field approximation, the coherent field and the average dyadic Green's function of the discrete random layer correspond to a homogeneous layer characterized by the effective wavenumber. The effective wavenumber is computed by neglecting boundary effects. Under the assumption that the layer thickness is greater than the mean free path, the effective wavenumber is that of an unbounded discrete random medium.

A3. In computing the Wigner transforms of the tetradic Green's function and the coherent dyadic correlation function, all cross terms are excluded. Consequently, the integral equation (103), derived under the effective field approximation, implies that the expression for the Wigner transform of the dyadic correlation function is equivalent to that obtained in the quasi-uniform field approximation.
A4. The scattering intensity operator is computed in the ladder approximation for the Bethe-Salpeter equation, while the Wigner-Fourier transform of the scattering intensity operator is computed by means of the on-shell approximation for the Fourier transform of the intensity operator.

A5. The weak surface correlation approximation is used for averaging over surface fluctuations.

The conventional radiative transfer equation has been obtained by further assuming that $K^{\prime \prime} \ll K^{\prime}$ as well as by neglecting the evanescent waves (far-field approximation).

The following conclusions can be drawn from our analysis.

1. The quasi-uniform field approximation has not been used explicitly. It is a consequence of the effective field approximation and the exclusion of the cross terms in the expressions for the tetradic Green's function and the coherent dyadic correlation function.

2. Assumptions A3 and A5, as well as the assumption that the layer thickness is greater than the mean free path, are required when dealing with a discrete random medium with a finite geometrical thickness and scattering boundaries.

3. If the layer has non-scattering boundaries, the main assumptions of the derivation are the effective field approximation and the on-shell approximation to the intensity operator. The validity of the former is discussed in detail in Appendix 2. The conclusion of this analysis is that the effective field approximation (with or without coherent potential) applies to sparse media when the common assumptions and approximations (uncorrelated particle positions, far-field approximation, and the Twersky approximation) are made. Its application to dense media is questionable; it is only in the low-frequency limit that it is justified. Up to this point, these assumptions are essentially equivalent to those in Ref. [1]. What is new is the on-shell approximation to the intensity operator. From a mathematical point of view, this approximation, which in principle should follow from the sparse medium assumptions, is a consequence of using the Wigner-transform method; without this approximation, the desired representation of the radiative transfer equation cannot be obtained.

\section{Appendix A. Boundary conditions for $\overline{\mathscr{C}}^{a a}\left(Z, p_{\perp}\right)$}

To derive the boundary condition at the lower boundary, we set $Z=0$ in Eq. (151). Then we find that $\overline{\mathscr{C}}^{++}\left(0, \mathbf{p}_{\perp}\right)$ and $\overline{\mathscr{C}}^{--}\left(0, \mathbf{p}_{\perp}\right)$ can be written as $\overline{\mathscr{C}}^{++}\left(0, \mathbf{p}_{\perp}\right)=\sum_{b= \pm} \overline{\mathscr{C}}_{b}^{++}\left(0, \mathbf{p}_{\perp}\right)$ and $\overline{\mathscr{C}}^{--}\left(0, \mathbf{p}_{\perp}\right)=\sum_{b= \pm} \overline{\mathscr{C}}_{b}^{--}\left(0, \mathbf{p}_{\perp}\right)$, respectively, with

$$
\overline{\mathscr{C}}_{b}^{++}\left(0, \mathbf{p}_{\perp}\right)=\int \overline{\mathcal{S}}^{1+1 b}\left(\mathbf{p}_{\perp}, \mathbf{p}_{1 \perp}, 0\right) \cdot \overline{\overline{\mathbf{X}}}^{b}\left(\mathbf{p}_{1 \perp}\right) \mathrm{d}^{2} \mathbf{p}_{1 \perp},
$$

$$
\begin{aligned}
\overline{\mathscr{C}}_{b}^{--}\left(0, \mathbf{p}_{\perp}\right)= & \int\left[\delta\left(\mathbf{p}_{1 \perp}-\mathbf{p}_{\perp}\right) \delta_{-b} \overline{\overline{\mathbf{I}}}_{\perp}^{--}\left(\mathbf{p}_{\perp}\right)\right. \\
& \left.+\overline{\overline{\mathcal{S}}}^{1-1 b}\left(\mathbf{p}_{\perp}, \mathbf{p}_{1 \perp}, 0\right)\right] \cdot \overline{\overline{\mathbf{X}}}^{b}\left(\mathbf{p}_{1 \perp}\right) \mathrm{d}^{2} \mathbf{p}_{1 \perp} .
\end{aligned}
$$

In Eqs. (187) and (188), the tetradic $\overline{\overline{\mathbf{X}}}^{b}\left(\mathbf{p}_{1 \perp}\right)$ is the result of integrating over $\mathbf{p}_{2 \perp}$ and $Z_{1}$. Our first goal is to establish relationships between $\overline{\mathscr{C}}_{b}^{++}\left(0, \mathbf{p}_{\perp}\right)$ and $\overline{\mathscr{C}}_{b}^{--}\left(0, \mathbf{p}_{\perp}\right)$ in the cases $b=+$ and $b=-$.

1. In the case $b=+$, Eqs. (187) and (188) read

$$
\begin{aligned}
& \overline{\mathscr{C}}_{+}^{++}\left(0, \mathbf{p}_{\perp}\right)=\int \overline{\overline{\mathcal{S}}}^{1+1+}\left(\mathbf{p}_{\perp}, \mathbf{p}_{1 \perp}, 0\right) \cdot \overline{\overline{\mathbf{X}}}^{+}\left(\mathbf{p}_{1 \perp}\right) \mathrm{d}^{2} \mathbf{p}_{1 \perp}, \\
& \overline{\mathscr{C}}_{+}^{--}\left(0, \mathbf{p}_{\perp}\right)=\int \overline{\mathcal{S}}^{1-1+}\left(\mathbf{p}_{\perp}, \mathbf{p}_{1 \perp}, 0\right) \cdot \overline{\overline{\mathbf{X}}}^{+}\left(\mathbf{p}_{1 \perp}\right) \mathrm{d}^{2} \mathbf{p}_{1 \perp} .
\end{aligned}
$$


On the other hand, from (cf. Eq. (65))

$\overline{\mathbf{S}}^{1+1+}=\overline{\mathbf{R}}_{01} \overline{\mathbf{S}}^{1-1+}$,

we get

$$
\begin{aligned}
& \overline{\mathbf{S}}^{1+1+}\left(\mathbf{k}_{\perp}, \mathbf{k}_{1 \perp}\right) \otimes \overline{\mathbf{S}}^{1+1+\star}\left(\mathbf{k}_{\perp}^{\prime}, \mathbf{k}_{1 \perp}^{\prime}\right) \\
& =\int\left[\overline{\mathbf{R}}_{01}\left(\mathbf{k}_{\perp}, \mathbf{k}_{2 \perp}\right) \otimes \overline{\mathbf{R}}_{01}^{\star}\left(\mathbf{k}_{\perp}^{\prime}, \mathbf{k}_{2 \perp}^{\prime}\right)\right] \\
& \quad \cdot\left[\overline{\mathbf{S}}^{1-1+}\left(\mathbf{k}_{2 \perp}, \mathbf{k}_{1 \perp}\right) \otimes \overline{\mathbf{S}}^{1-1+\star}\left(\mathbf{k}_{2 \perp}^{\prime}, \mathbf{k}_{1 \perp}^{\prime}\right)\right] \mathrm{d}^{2} \mathbf{k}_{2 \perp}^{\prime} \mathrm{d}^{2} \mathbf{k}_{2 \perp} .
\end{aligned}
$$

Taking the average over surface fluctuations under the weak surface correlation approximation (the tetradics $\overline{\mathbf{R}}_{01} \otimes \overline{\mathbf{R}}_{01}^{\star}$ and $\overline{\mathbf{S}}^{1-1+} \otimes \overline{\mathbf{S}}^{1-1+\star}$ are assumed to be weakly correlated on the rough surface), we obtain

$$
\begin{aligned}
& \left\langle\overline{\mathbf{S}}^{1+1+}\left(\mathbf{k}_{\perp}, \mathbf{k}_{1 \perp}\right) \otimes \overline{\mathbf{S}}^{1+1+\star}\left(\mathbf{k}_{\perp}^{\prime}, \mathbf{k}_{1 \perp}^{\prime}\right)\right\rangle_{S} \\
& =\int\left\langle\overline{\mathbf{R}}_{01}\left(\mathbf{k}_{\perp}, \mathbf{k}_{2 \perp}\right) \otimes \overline{\mathbf{R}}_{01}^{\star}\left(\mathbf{k}_{\perp}^{\prime}, \mathbf{k}_{2 \perp}^{\prime}\right)\right\rangle_{S} \\
& \quad \cdot\left\langle\overline{\mathbf{S}}^{1-1+}\left(\mathbf{k}_{2 \perp}, \mathbf{k}_{1 \perp}\right) \otimes \overline{\mathbf{S}}^{1-1+\star}\left(\mathbf{k}_{2 \perp}^{\prime}, \mathbf{k}_{1 \perp}^{\prime}\right)\right\rangle_{S} \mathrm{~d}^{2} \mathbf{k}_{2 \perp}^{\prime} \mathrm{d}^{2} \mathbf{k}_{2 \perp} .
\end{aligned}
$$

In Eq. (193), we make the changes of variables

$\mathbf{k}_{\perp}=\mathbf{p}_{\perp}+\frac{\mathbf{P}_{\perp}}{2}, \quad \mathbf{k}_{\perp}^{\prime}=\mathbf{p}_{\perp}-\frac{\mathbf{P}_{\perp}}{2}$,

$\mathbf{k}_{1 \perp}=\mathbf{p}_{1 \perp}+\frac{\mathbf{P}_{1 \perp}}{2}, \quad \mathbf{k}_{1 \perp}^{\prime}=\mathbf{p}_{1 \perp}-\frac{\mathbf{P}_{1 \perp}}{2}$,

$\mathbf{k}_{2 \perp}=\mathbf{p}_{2 \perp}+\frac{\mathbf{P}_{2 \perp}}{2}, \quad \mathbf{k}_{2 \perp}^{\prime}=\mathbf{p}_{2 \perp}-\frac{\mathbf{P}_{2 \perp}}{2} ;$

then, in using $\mathrm{d}^{2} \mathbf{k}_{2 \perp}^{\prime} \mathrm{d}^{2} \mathbf{k}_{2 \perp}=\mathrm{d}^{2} \mathbf{p}_{2 \perp} \mathrm{d}^{2} \mathbf{P}_{2 \perp}$ (the Jacobian of the transformation $\left(\mathbf{k}_{2 \perp}, \mathbf{k}_{2 \perp}^{\prime}\right) \rightarrow\left(\mathbf{p}_{2 \perp}, \mathbf{P}_{2 \perp}\right)$ is readily seen to be unity), we find

$$
\begin{aligned}
\overline{\overline{\mathcal{S}}}^{1+1+}\left(\mathbf{p}_{\perp}, \mathbf{p}_{1 \perp}, \mathbf{P}_{\perp}\right)= & \int \overline{\overline{\mathcal{R}}}_{01}\left(\mathbf{p}_{\perp}, \mathbf{p}_{2 \perp}, \mathbf{P}_{\perp}\right) \\
& \cdot \overline{\overline{\mathcal{S}}}^{1-1+}\left(\mathbf{p}_{2 \perp}, \mathbf{p}_{1 \perp}, \mathbf{P}_{\perp}\right) \mathrm{d}^{2} \mathbf{p}_{2 \perp},
\end{aligned}
$$

where, the tetradic reflection operator $\overline{\overline{\mathcal{R}}}_{01}$ is defined as in Eq. (75), i.e.,

$$
\begin{aligned}
\overline{\mathcal{\mathcal { R }}}_{01}\left(\mathbf{p}_{\perp}, \mathbf{p}_{1 \perp}, \mathbf{P}_{\perp}\right) & =\frac{(2 \pi)^{2}}{A}\left\langle\overline{\mathbf{R}}_{01}\left(\mathbf{p}_{\perp}+\frac{\mathbf{P}_{\perp}}{2}, \mathbf{p}_{1 \perp}+\frac{\mathbf{P}_{\perp}}{2}\right)\right. \\
& \left.\otimes \overline{\mathbf{R}}_{01}^{\star}\left(\mathbf{p}_{\perp}-\frac{\mathbf{P}_{\perp}}{2}, \mathbf{p}_{1 \perp}-\frac{\mathbf{P}_{\perp}}{2}\right)\right\rangle_{S}
\end{aligned}
$$

and the tetradic $\overline{\overline{\mathcal{S}}}^{1 a 1 b}$ is defined in a similar manner. Setting $\mathbf{P}_{\perp}=0$ in Eq. (197), and using Eqs. (189) and (190) gives

$$
\overline{\mathscr{C}}_{+}^{++}\left(0, \mathbf{p}_{\perp}\right)=\int \overline{\overline{\mathcal{R}}}_{01}\left(\mathbf{p}_{\perp}, \mathbf{p}_{1 \perp}, 0\right) \cdot \overline{\mathscr{C}}_{+}^{--}\left(0, \mathbf{p}_{1 \perp}\right) \mathrm{d}^{2} \mathbf{p}_{1 \perp} .
$$

2. In the case $b=-$, Eqs. (187) and (188) take the form

$$
\begin{aligned}
\overline{\mathscr{C}}_{-}^{++}\left(0, \mathbf{p}_{\perp}\right)= & \int \overline{\overline{\mathcal{S}}}^{1+1-}\left(\mathbf{p}_{\perp}, \mathbf{p}_{1 \perp}, 0\right) \cdot \overline{\overline{\mathbf{X}}}^{-}\left(\mathbf{p}_{1 \perp}\right) \mathrm{d}^{2} \mathbf{p}_{1 \perp}, \\
\overline{\mathscr{C}}_{-}^{--}\left(0, \mathbf{p}_{\perp}\right)= & \int\left[\delta\left(\mathbf{p}_{1 \perp}-\mathbf{p}_{\perp}\right) \overline{\overline{\mathbf{I}}}_{\perp}^{--}\left(\mathbf{p}_{\perp}\right)\right. \\
& \left.+\overline{\overline{\mathcal{S}}}^{1-1-}\left(\mathbf{p}_{\perp}, \mathbf{p}_{1 \perp}, 0\right)\right] \cdot \overline{\overline{\mathbf{X}}}^{-}\left(\mathbf{p}_{1 \perp}\right) \mathrm{d}^{2} \mathbf{p}_{1 \perp} .
\end{aligned}
$$

Now, from (cf. Eq. (66))

$$
\overline{\mathbf{S}}^{1+1-}=\overline{\mathbf{R}}_{01}\left(\overline{\mathbf{I}}_{\perp}^{1-1-}+\overline{\mathbf{S}}^{1-1-}\right),
$$

the approximation

$$
\left(\overline{\mathbf{I}}_{\perp}^{1-1-}+\overline{\mathbf{S}}^{1-1-}\right) \otimes\left(\overline{\mathbf{I}}_{\perp}^{1-1-}+\overline{\mathbf{S}}^{1-1-\star}\right)
$$

$$
\approx \overline{\mathbf{I}}_{\perp}^{1-1-} \otimes \overline{\mathbf{I}}_{\perp}^{1-1-}+\overline{\mathbf{S}}^{1-1-} \otimes \overline{\mathbf{S}}^{1-1-\star},
$$

which is similar to the approximation we used while computing the Wigner transform of the dyadic Green's function, and the weak surface correlation approximation, we get

$$
\begin{aligned}
\left\langle\overline{\mathbf{S}}^{1+1-}\left(\mathbf{k}_{\perp}, \mathbf{k}_{1 \perp}\right) \otimes \overline{\mathbf{S}}^{1+1-\star}\left(\mathbf{k}_{\perp}^{\prime}, \mathbf{k}_{1 \perp}^{\prime}\right)\right\rangle_{S} \\
=\int\left\langle\overline{\mathbf{R}}_{01}\left(\mathbf{k}_{\perp}, \mathbf{k}_{2 \perp}\right) \otimes \overline{\mathbf{R}}_{01}^{\star}\left(\mathbf{k}_{\perp}^{\prime}, \mathbf{k}_{2 \perp}^{\prime}\right)\right\rangle_{S} \\
\quad \cdot\left[\overline{\mathbf{I}}_{\perp}^{1-1-}\left(\mathbf{k}_{2 \perp}, \mathbf{k}_{1 \perp}\right) \otimes \overline{\mathbf{I}}_{\perp}^{1-1-}\left(\mathbf{k}_{2 \perp}^{\prime}, \mathbf{k}_{1 \perp}^{\prime}\right)\right. \\
\left.\quad+\left\langle\overline{\mathbf{S}}^{1-1-}\left(\mathbf{k}_{2 \perp}, \mathbf{k}_{1 \perp}\right) \otimes \overline{\mathbf{S}}^{1-1-\star}\left(\mathbf{k}_{2 \perp}^{\prime}, \mathbf{k}_{1 \perp}^{\prime}\right)\right\rangle_{S}\right] \mathrm{d}^{2} \mathbf{k}_{2 \perp}^{\prime} \mathrm{d}^{2} \mathbf{k}_{2 \perp} .
\end{aligned}
$$

Using (cf. Eq. (71))

$\overline{\mathbf{I}}_{\perp}^{1-1-}\left(\mathbf{k}_{2 \perp}, \mathbf{k}_{1 \perp}\right)=\delta\left(\mathbf{k}_{2 \perp}-\mathbf{k}_{1 \perp}\right) \overline{\mathbf{I}}_{\perp}^{-}\left(\mathbf{k}_{1 \perp}\right)$,

and making the changes of variables (194)-(196) in Eq. (204), we obtain

$$
\begin{aligned}
\overline{\overline{\mathcal{S}}}^{1+1-}\left(\mathbf{p}_{\perp}, \mathbf{p}_{1 \perp}, \mathbf{P}_{\perp}\right) \\
=\int \overline{\overline{\mathcal{R}}}_{01}\left(\mathbf{p}_{\perp}, \mathbf{p}_{2 \perp}, \mathbf{P}_{\perp}\right) \\
\quad \cdot\left[\delta\left(\mathbf{p}_{2 \perp}-\mathbf{p}_{1 \perp}\right) \overline{\mathbf{I}}_{\perp}\left(\mathbf{p}_{1 \perp}+\frac{\mathbf{P}_{\perp}}{2}\right) \otimes \overline{\mathbf{I}}_{\perp}\left(\mathbf{p}_{1 \perp}-\frac{\mathbf{P}_{\perp}}{2}\right)\right. \\
\left.\quad+\overline{\mathcal{\mathcal { S }}}^{1-1-}\left(\mathbf{p}_{2 \perp}, \mathbf{p}_{1 \perp}, \mathbf{P}_{\perp}\right)\right] \mathrm{d}^{2} \mathbf{p}_{2 \perp} .
\end{aligned}
$$

Setting $\mathbf{P}_{\perp}=0$ in Eq. (205) and taking into account Eqs. (200) and (201) gives, as before,

$$
\overline{\mathscr{C}}_{-}^{++}\left(0, \mathbf{p}_{\perp}\right)=\int \overline{\overline{\mathcal{R}}}_{01}\left(\mathbf{p}_{\perp}, \mathbf{p}_{1 \perp}, 0\right) \cdot \overline{\mathscr{C}}_{-}^{--}\left(0, \mathbf{p}_{1 \perp}\right) \mathrm{d}^{2} \mathbf{p}_{1 \perp}
$$

From Eqs. (199) and (206), we infer that the boundary condition at $Z=0$ is

$$
\overline{\mathscr{C}}^{++}\left(0, \mathbf{p}_{\perp}\right)=\int \overline{\overline{\mathcal{R}}}_{01}\left(\mathbf{p}_{\perp}, \mathbf{p}_{1 \perp}, 0\right) \cdot \overline{\mathscr{C}}^{--}\left(0, \mathbf{p}_{1 \perp}\right) \mathrm{d}^{2} \mathbf{p}_{1 \perp} .
$$

To derive the boundary condition at $Z=H$ we use Eqs. (67), (68), and (72), and proceed analogously. We obtain

$\overline{\mathscr{C}}^{--}\left(H, \mathbf{p}_{\perp}\right)=\int \overline{\overline{\mathcal{R}}}_{21}\left(\mathbf{p}_{\perp}, \mathbf{p}_{1 \perp}, 0\right) \cdot \overline{\mathscr{C}}^{++}\left(H, \mathbf{p}_{1 \perp}\right) \mathrm{d}^{2} \mathbf{p}_{1 \perp}$,

where the tetradic reflection operator $\overline{\overline{\mathcal{R}}}_{21}\left(\mathbf{p}_{\perp}, \mathbf{p}_{1 \perp}, 0\right)$ at the upper boundary is given by Eq. (135).

\section{Appendix B. Effective field approximation}

In this appendix we analyze under what conditions the average dyadic Green's function for a discrete random medium can be approximated by the dyadic Green's function for a homogeneous medium with the effective propagation constant. For simplicity, the medium is assumed to be unbounded.

Taking the Fourier transform of the Dyson equation for the average dyadic Green's function $\langle\overline{\mathbf{G}}\rangle=\overline{\mathbf{G}}_{0}+\overline{\mathbf{G}}_{0} \overline{\mathbf{M}}\langle\overline{\mathbf{G}}\rangle$, under the assumption that for a statistically homogeneous medium, the mass operator and the average dyadic Green's function are translation invariant, i.e., $\overline{\mathbf{M}}\left(\mathbf{r}, \mathbf{r}^{\prime}\right)=\overline{\mathbf{M}}\left(\mathbf{r}-\mathbf{r}^{\prime}\right)$ and $\left\langle\overline{\mathbf{G}}\left(\mathbf{r}, \mathbf{r}^{\prime}\right)\right\rangle=\left\langle\overline{\mathbf{G}}\left(\mathbf{r}-\mathbf{r}^{\prime}\right)\right\rangle$, respectively, we are led to the dispersion equation [11]

$\operatorname{det}\left[\left(p^{2}-k_{1}^{2}\right)(\overline{\mathbf{I}}-\widehat{\mathbf{p}} \otimes \widehat{\mathbf{p}})-k_{1}^{2} \widehat{\mathbf{p}} \otimes \widehat{\mathbf{p}}-\overline{\mathbf{M}}_{p}(\mathbf{p})\right]=0$

for the propagation direction $\widehat{\mathbf{p}}$, where $\overline{\mathbf{G}}_{0}\left(\mathbf{r}, \mathbf{r}^{\prime}\right)$ is the free-space dyadic Green's function and $\overline{\mathbf{M}}_{p}(\mathbf{p})$ is the Fourier transform of the mass operator. In a statistically isotropic medium, e.g., a medium consisting of spherical particles or randomly oriented particles 
with a plane of symmetry, we assume that the mass operator is of the form

$\overline{\mathbf{M}}_{p}(\mathbf{p})=M_{p \mathrm{~L}}(p) \widehat{\mathbf{p}} \otimes \widehat{\mathbf{p}}+M_{p \mathrm{~T}}(p)(\overline{\mathbf{I}}-\widehat{\mathbf{p}} \otimes \widehat{\mathbf{p}})$,

in which case Eq. (208) yields the dispersion equations for the longitudinal modes

$k_{1}^{2}+M_{p \mathrm{~L}}(p)=0$,

and the transverse modes

$p^{2}=k_{1}^{2}+M_{p \mathrm{~T}}(p)$.

The assumption (209) reflects the statistical isotropy of the medium, i.e., the effective wavenumber $K$ solving Eqs. (210) or (211) does not depend on the propagation direction $\widehat{\mathbf{p}}$.

We are now well prepared to state the so-called homogenization theorem [18]. For a statistically isotropic medium, assume the following:

A1. The mass operator is as in Eq. (209).

A2. The equations $k_{1}^{2}+M_{p \mathrm{~L}}(p)=0$ and $k_{1}^{2}+M_{p \mathrm{~T}}(p)=0$ have no solutions.

A3. From the set of solutions of the dispersion equation for the transverse modes $p^{2}=k_{1}^{2}+M_{p \mathrm{~T}}(p)$, consider only the solution $K$ with the smallest positive imaginary part.

Then, the average dyadic Green's function $\langle\overline{\mathbf{G}}\rangle$ can be approximated by the dyadic Green's function of an infinite homogeneous medium with wavenumber $K$,

$\overline{\mathbf{G}}_{\mathrm{K}}\left(\mathbf{r}, \mathbf{r}^{\prime}\right)=\overline{\mathbf{G}}_{\mathrm{K}}(\mathbf{R})=\left(\overline{\mathbf{I}}+\frac{1}{K^{2}} \nabla \otimes \nabla\right) \frac{\mathrm{e}^{\mathrm{j} K R}}{4 \pi R}, \quad \mathbf{R}=\mathbf{r}-\mathbf{r}^{\prime}$,

that is

$\langle\overline{\mathbf{G}}\rangle \approx \overline{\mathbf{G}}_{\mathrm{K}}$.

Let us prove this result. From $\langle\overline{\mathbf{G}}\rangle^{-1}=\overline{\mathbf{G}}_{0}^{-1}-\overline{\mathbf{M}}$ and Assumption A1, the Fourier transform of the average dyadic Green's function is given by

$$
\begin{aligned}
\overline{\mathbf{G}}_{p}(\mathbf{p}) & =\left[\overline{\mathbf{G}}_{0 p}^{-1}(\mathbf{p})-\overline{\mathbf{M}}_{p}(\mathbf{p})\right]^{-1} \\
& =\frac{1}{\left(p^{2}-k_{1}^{2}\right)-M_{p \mathrm{~T}}(p)}(\overline{\mathbf{I}}-\widehat{\mathbf{p}} \otimes \widehat{\mathbf{p}})-\frac{1}{k_{1}^{2}+M_{p \mathrm{~L}}(p)} \widehat{\mathbf{p}} \otimes \widehat{\mathbf{p}},
\end{aligned}
$$

where

$\overline{\mathbf{G}}_{0 p}(\mathbf{p})=\frac{1}{p^{2}-k_{1}^{2}}(\overline{\mathbf{I}}-\widehat{\mathbf{p}} \otimes \widehat{\mathbf{p}})-\frac{1}{k_{1}^{2}} \widehat{\mathbf{p}} \otimes \widehat{\mathbf{p}}$

is the Fourier transform of $\overline{\mathbf{G}}_{0}\left(\mathbf{r}, \mathbf{r}^{\prime}\right)$. Setting

$k_{p \mathrm{~T}}^{2}(p)=k_{1}^{2}+M_{p \mathrm{~T}}(p)$,

$k_{p \mathrm{~L}}^{2}(p)=k_{1}^{2}+M_{p \mathrm{~L}}(p)$

in Eq. (214), we obtain

$$
\begin{aligned}
\overline{\mathbf{G}}_{p}(\mathbf{p})= & {\left[\overline{\mathbf{I}}-\frac{p^{2}}{k_{p \mathrm{~T}}^{2}(p)} \widehat{\mathbf{p}} \otimes \widehat{\mathbf{p}}\right] \frac{1}{p^{2}-k_{p \mathrm{~T}}^{2}(p)} } \\
& +\frac{1}{k_{p \mathrm{~T}}^{2}(p)} \widehat{\mathbf{p}} \otimes \widehat{\mathbf{p}}-\frac{1}{k_{p \mathrm{~L}}^{2}(p)} \widehat{\mathbf{p}} \otimes \widehat{\mathbf{p}} .
\end{aligned}
$$

Using the relations

$$
\begin{aligned}
& \nabla \otimes \nabla \mathrm{e}^{\mathrm{j} \mathbf{p} \cdot \mathbf{r}}=-p^{2} \mathrm{e}^{\mathrm{j} \mathbf{p} \cdot \mathbf{r}} \widehat{\mathbf{p}} \otimes \widehat{\mathbf{p}}, \\
& \int \mathrm{e}^{\mathrm{j} \mathbf{p} \cdot \mathbf{R}} \mathrm{d}^{2} \widehat{\mathbf{p}}=4 \pi \frac{\sin (p R)}{p R},
\end{aligned}
$$

and

$\int_{-\infty}^{\infty} g(x) \mathrm{e}^{\mathrm{j} x} \mathrm{~d} x=2 \mathrm{j} \int_{0}^{\infty} g(x) \sin x \mathrm{~d} x$, for $g(-x)=-g(x)$,

and supposing that $k_{p \mathrm{~T}}^{2}(-p)=k_{p \mathrm{~T}}^{2}(p)$ and $k_{p \mathrm{~L}}^{2}(-p)=k_{p \mathrm{~L}}^{2}(p)$, we find that in the coordinate space, the average dyadic Green's function is

$$
\begin{aligned}
\langle\overline{\mathbf{G}}(\mathbf{R})\rangle & =\frac{1}{4 \pi^{2} \mathrm{j}} \int_{-\infty}^{\infty}\left[\overline{\mathbf{I}}+\frac{1}{k_{p \mathrm{~T}}^{2}(p)} \nabla \otimes \nabla\right] \frac{p}{p^{2}-k_{p \mathrm{~T}}^{2}(p)} \frac{\mathrm{e}^{\mathrm{j} p R}}{R} \mathrm{~d} p \\
& -\frac{1}{4 \pi^{2} \mathrm{j}} \nabla \otimes \nabla \int_{-\infty}^{\infty}\left[\frac{1}{k_{p \mathrm{~T}}^{2}(p)}-\frac{1}{k_{p \mathrm{~L}}^{2}(p)}\right] \frac{\mathrm{e}^{\mathrm{j} p R}}{p R} \mathrm{~d} p .
\end{aligned}
$$

The above integrals are computed by using the residue theorem. By Assumption A2, there are no poles corresponding to $k_{p \mathrm{~T}}(p)=0$ and $k_{p \mathrm{~L}}(p)=0$. Hence, in the first integral, written as

$$
\int_{-\infty}^{\infty} \overline{\mathbf{I}} \frac{p}{p^{2}-k_{p \mathrm{~T}}^{2}(p)} \frac{\mathrm{e}^{\mathrm{j} p R}}{R} \mathrm{~d} p+\nabla \otimes \nabla \int_{-\infty}^{\infty} \frac{1}{k_{p \mathrm{~T}}^{2}(p)} \frac{p}{p^{2}-k_{p \mathrm{~T}}^{2}(p)} \frac{\mathrm{e}^{\mathrm{j} p R}}{R} \mathrm{~d} p,
$$

the remaining poles are the solutions of the equation $p^{2}-k_{p \mathrm{~T}}^{2}(p)=$ 0 , that is, the solutions of the dispersion equation for the transverse modes $p^{2}=k_{1}^{2}+M_{p \mathrm{~T}}(p)$. If in view of Assumption A3, we retain only the solution $K$ with the smallest positive imaginary part, we obtain

$\int_{-\infty}^{\infty} \frac{p}{p^{2}-k_{p \mathrm{~T}}^{2}(p)} \frac{\mathrm{e}^{\mathrm{j} p R}}{R} \mathrm{~d} p=\pi \mathrm{j} \frac{\mathrm{e}^{\mathrm{j} K R}}{R}$

and

$\int_{-\infty}^{\infty} \frac{1}{k_{p \mathrm{~T}}^{2}(p)} \frac{p}{p^{2}-k_{p \mathrm{~T}}^{2}(p)} \frac{\mathrm{e}^{\mathrm{j} p R}}{R} \mathrm{~d} p=\pi \mathrm{j} \frac{1}{K^{2}} \frac{\mathrm{e}^{\mathrm{j} K R}}{R}$

whence

$\frac{1}{4 \pi^{2} \mathrm{j}} \int_{-\infty}^{\infty}\left[\overline{\mathbf{I}}+\frac{1}{k_{p \mathrm{~T}}^{2}(p)} \nabla \otimes \nabla\right] \frac{p}{p^{2}-k_{p \mathrm{~T}}^{2}(p)} \frac{\mathrm{e}^{\mathrm{j} p R}}{R} \mathrm{~d} p$

$=\left(\overline{\mathbf{I}}+\frac{1}{K^{2}} \nabla \otimes \nabla\right) \frac{\mathrm{e}^{\mathrm{j} K R}}{4 \pi R}$.

Note that the solution $K$ with the smallest positive imaginary part has the largest exponential factor $\exp \left(-K^{\prime \prime} R\right)$; the solutions with larger positive imaginary parts are strongly attenuated in the medium. In the second integral, written as

$\int_{-\infty}^{\infty}\left[\frac{1}{k_{p \mathrm{~T}}^{2}(p)}-\frac{1}{k_{p \mathrm{~L}}^{2}(p)}\right] \frac{\mathrm{e}^{\mathrm{j} p R}}{p R} \mathrm{~d} p$

we have a single pole at $p=0$. Choosing a semicircular integration contour in the counter-clockwise direction around the pole $p=0$, we obtain

$\int_{-\infty}^{\infty}\left[\frac{1}{k_{p \mathrm{~T}}^{2}(p)}-\frac{1}{k_{p \mathrm{~L}}^{2}(p)}\right] \frac{\mathrm{e}^{\mathrm{j} p R}}{p R} \mathrm{~d} p=-\pi \mathrm{j}\left[\frac{1}{k_{p \mathrm{~T}}^{2}(0)}-\frac{1}{k_{p \mathrm{~L}}^{2}(0)}\right] \frac{1}{R}$.

From

$\nabla \otimes \nabla\left(\frac{1}{R}\right)=\frac{2}{R^{3}} \widehat{\mathbf{R}} \otimes \widehat{\mathbf{R}}-\frac{1}{R^{3}}(\overline{\mathbf{I}}-\widehat{\mathbf{R}} \otimes \widehat{\mathbf{R}})=(-\overline{\mathbf{I}}+3 \widehat{\mathbf{R}} \otimes \widehat{\mathbf{R}}) \frac{1}{R^{3}}$,

we then get

$$
\begin{aligned}
& -\frac{1}{4 \pi^{2} \mathrm{j}} \nabla \otimes \nabla \int_{-\infty}^{\infty}\left[\frac{1}{k_{p \mathrm{~T}}^{2}(p)}-\frac{1}{k_{p \mathrm{~L}}^{2}(p)}\right] \frac{\mathrm{e}^{\mathrm{j} p R}}{p R} \mathrm{~d} p \\
& =\left[\frac{1}{k_{p T}^{2}(0)}-\frac{1}{k_{p L}^{2}(0)}\right](-\overline{\mathbf{I}}+3 \widehat{\mathbf{R}} \otimes \widehat{\mathbf{R}}) \frac{1}{4 \pi R^{3}} .
\end{aligned}
$$


In the effective and quasi-crystalline approximations, it has been shown that in the low frequency limit, i.e., $p \rightarrow 0$, we have $M_{p \mathrm{~L}}(p)=M_{p \mathrm{~T}}(p)=M_{p}$, so that the mass operator is of the form $\overline{\mathbf{M}}_{p}(\mathbf{p})=M_{p} \overline{\mathbf{I}}[11]$. Consequently, $k_{p \mathrm{~T}}^{2}(0)=k_{p \mathrm{~L}}^{2}(0)$, and the second integral in the expression for $\langle\overline{\mathbf{G}}(\mathbf{R})\rangle$ vanishes. From Eq. (222) we then find that the average dyadic Green's function is given by Eq. (212). Thus, the approximation $\langle\overline{\mathbf{G}}\rangle \approx \overline{\mathbf{G}}_{\mathrm{K}}$ is valid for a statistically isotropic medium when the mass operator is as in Eq. (209), the longitudinal modes are neglected, and the transverse mode with the smallest positive imaginary part is considered.

The main assumption in proving Eq. (213) is the representation (209) for the mass operator. However, in general, the isotropy condition is of the form $\overline{\mathbf{M}}_{p}(\mathbf{p})=\overline{\mathbf{M}}_{p}(p)$, or more restrictively but still more generally as in Eq. (209) of the form $\overline{\mathbf{M}}_{p}(\mathbf{p})=M_{p \mathrm{~L}}(p) \widehat{\mathbf{p}} \otimes \widehat{\mathbf{p}}+$ $\overline{\mathbf{M}}_{p \mathrm{~T}}(p)$, where $\overline{\mathbf{M}}_{p \mathrm{~T}}$ is the transverse component of $\overline{\mathbf{M}}_{p}$. Unfortunately, in these two cases we have not been able to prove the homogenization theorem.

In the following we analyze the applicability of the homogenization theorem to the cases discussed in Ref. [11].

\section{Effective field approximation or Foldy's approximation}

The Foldy approximation applies to sparse media and relies on the Foldy equation for the total field $\mathbf{E}=\mathbf{E}_{0}+\sum_{i} \overline{\mathbf{G}}_{0} \overline{\mathbf{T}}_{i} \mathbf{E}_{\text {exci }}$, where $\mathbf{E}_{\text {exci }}$ is the field exciting particle $i$. Taking the configuration average of this integral equation under the assumption that the positions of the particles are statistically independent, employing the first-order closure relation or the Foldy approximation $\left\langle\mathbf{E}_{\text {exci }}\right\rangle_{i}=$ $\langle\mathbf{E}\rangle$, where $\left\langle\mathbf{E}_{\text {exci }}\right\rangle_{i}$ is the conditional probability of the exciting field with the position of particle $i$ held fixed, and using the fact that $\overline{\mathbf{T}}_{i}$ is a translational dyadic, i.e., $\overline{\mathbf{T}}_{i}\left(\mathbf{r}, \mathbf{r}^{\prime}\right)=\overline{\mathbf{T}}\left(\mathbf{r}-\mathbf{R}_{i}, \mathbf{r}^{\prime}-\mathbf{R}_{i}\right)$, it is found that in the Fourier space, the mass operator is given by

$\overline{\mathbf{M}}_{p}(\mathbf{p})=n_{0} \overline{\mathbf{T}}_{p}(\mathbf{p}, \mathbf{p})$,

where $\overline{\mathbf{T}}_{p}\left(\mathbf{p}, \mathbf{p}^{\prime}\right)$ is the Fourier transform of $\overline{\mathbf{T}}\left(\mathbf{r}, \mathbf{r}^{\prime}\right)$ (the transition operator for a particle centered at the origin of the coordinate system). The dyadic $\overline{\mathbf{T}}_{p}\left(\mathbf{p}, \mathbf{p}^{\prime}\right)$ satisfies the Lippmann-Schwinger equation in the Fourier domain

$$
\begin{aligned}
\overline{\mathbf{T}}_{p}\left(\mathbf{p}, \mathbf{p}^{\prime}\right)= & \overline{\mathbf{U}}_{0 p}\left(\mathbf{p}, \mathbf{p}^{\prime}\right)+\frac{1}{(2 \pi)^{3}} \int \overline{\mathbf{U}}_{0 p}\left(\mathbf{p}, \mathbf{p}^{\prime \prime}\right) \\
& \cdot \overline{\mathbf{G}}_{0 p}\left(\mathbf{p}^{\prime \prime}\right) \cdot \overline{\mathbf{T}}_{p}\left(\mathbf{p}^{\prime \prime}, \mathbf{p}^{\prime}\right) \mathrm{d}^{3} \mathbf{p}^{\prime \prime},
\end{aligned}
$$

where $\overline{\mathbf{U}}_{0 p}\left(\mathbf{p}, \mathbf{p}^{\prime}\right)$ is the Fourier transform of the scattering potential of a particle centered at the origin of the coordinate system $\overline{\mathbf{U}}_{0}\left(\mathbf{r}, \mathbf{r}^{\prime}\right)=U_{0}(\mathbf{r}) \delta\left(\mathbf{r}-\mathbf{r}^{\prime}\right) \overline{\mathbf{I}}$ with $U_{0}(\mathbf{r})=k_{1}^{2}\left(\mathrm{~m}^{2}-1\right) \Theta(\mathbf{r})$. For spherical particles, the following representation for $\overline{\mathbf{T}}_{p}(\mathbf{p}, \mathbf{p})$ has been derived in [19]:

$$
\overline{\mathbf{T}}_{p}(\mathbf{p}, \mathbf{p})=T_{p \mathrm{~L}}(p) \widehat{\mathbf{p}} \otimes \widehat{\mathbf{p}}+T_{p \mathrm{~T}}(p)(\overline{\mathbf{I}}-\widehat{\mathbf{p}} \otimes \widehat{\mathbf{p}}),
$$

where

$T_{p \mathrm{~L}}(p)=V_{0} k_{1}^{2} \delta \mathrm{m}\left\{\sum_{n \geq 0} \frac{3 n(n+1)(2 n+1) \Delta \mathrm{m}}{\varphi_{n}\left(k_{\mathrm{p}} a\right)-\mathrm{m}^{2} \varphi_{n}^{(1)}\left(k_{1} a\right)}\left[\frac{j_{n}(p a)}{p a}\right]^{2}+1\right\}$

and

$T_{p \mathrm{~T}}(p)=T_{p 2}(p)+T_{p 3}(p)$,

with

$$
\begin{aligned}
T_{p 2}(p) & =V_{0} k_{1}^{2} \delta \mathrm{m}\left\{\left[1+\frac{p^{4} \Delta \mathrm{m}}{\left(p^{2}-k_{\mathrm{p}}^{2}\right)^{2}}\right] \mathcal{S}(p a)\right. \\
& -\frac{1}{2} \mathrm{~m}^{2} \frac{p^{2}-k_{1}^{2}}{p^{2}-k_{\mathrm{p}}^{2}}[\mathcal{S}(p a)-1]
\end{aligned}
$$

$$
\begin{aligned}
& +\frac{3}{2} \sum_{n \geq 1}(2 n+1)\left[\frac{\Delta \mathrm{m} R_{n}^{2}\left(p a, k_{\mathrm{p}} a\right)}{\varphi_{n}\left(k_{\mathrm{p}} a\right)-\mathrm{m}^{2} \varphi_{n}^{(1)}\left(k_{1} a\right)}\right. \\
& \left.\left.-\frac{p^{4} \triangle \mathrm{m}}{\left(p^{2}-k_{\mathrm{p}}^{2}\right)^{2}} \varphi_{n}\left(k_{\mathrm{p}} a\right)\right] J_{n}(p a, p a)\right\}
\end{aligned}
$$

and

$$
\begin{aligned}
T_{p 3}(p)= & V_{0} k_{1}^{2} \Delta \mathrm{m}\left\{\frac{p^{2} k_{1}^{2} \Delta \mathrm{m}}{\left(p^{2}-k_{\mathrm{p}}^{2}\right)^{2}} \mathcal{S}(p a)-\frac{1}{2} \frac{p^{2}-k_{1}^{2}}{p^{2}-k_{\mathrm{p}}^{2}}[\mathcal{S}(p a)-1]\right. \\
& +\frac{3}{2} \sum_{n \geq 1}(2 n+1)\left[\frac{\left(k_{1} a\right)^{2}(p a)^{2} \triangle \mathrm{m} S_{n}^{2}\left(p a, k_{\mathrm{p}} a\right)}{\varphi_{n}\left(k_{\mathrm{p}}\right)-\varphi_{n}^{(1)}\left(k_{1}\right)}\right. \\
& \left.\left.-\frac{p^{2} k_{1}^{2} \Delta \mathrm{m}}{\left(p^{2}-k_{\mathrm{p}}^{2}\right)^{2}} \varphi_{n}\left(k_{\mathrm{p}} a\right)\right] J_{n}(p a, p a)\right\}
\end{aligned}
$$

In Eqs. (231) and (232), $V_{0}=(4 / 3) \pi a^{3}$ is the volume of the spherical particle of radius $a, \Delta \mathrm{m}$ and $\delta \mathrm{m}$ are given, respectively, by $\Delta \mathrm{m}=\mathrm{m}^{2}-1$ and $\delta \mathrm{m}=1-1 / \mathrm{m}^{2}$, where $\mathrm{m}=k_{\mathrm{p}} / k_{1}$ is the relative refractive index of the particle, while the auxiliary functions $J_{n}, S_{n}$, $R_{n}$, and $\mathcal{S}$ are given by

$$
\begin{aligned}
& J_{n}\left(p_{1} a, p_{2} a\right)=\frac{j_{n}\left(p_{1} a\right)}{p_{1} a} \frac{j_{n}\left(p_{2} a\right)}{p_{2} a}, \\
& S_{n}\left(p_{1} a, p_{2} a\right)=\frac{\varphi_{n}\left(p_{1} a\right)-\varphi_{n}\left(p_{2} a\right)}{\left(p_{1} a\right)^{2}-\left(p_{2} a\right)^{2}}, \\
& R_{n}\left(p_{1} a, p_{2} a\right)=\frac{\left(p_{1} a\right)^{2} \varphi_{n}\left(p_{2} a\right)-\left(p_{2} a\right)^{2} \varphi_{n}\left(p_{1} a\right)}{\left(p_{1} a\right)^{2}-\left(p_{2} a\right)^{2}},
\end{aligned}
$$

$\mathcal{S}(x)=\frac{3}{2 x^{2}}\left[1-j_{0}(2 x)\right]$,

with

$\varphi_{n}(p a)=\frac{\left[p a j_{n}(p a)\right]^{\prime}}{j_{n}(p a)}$ and $\varphi_{n}^{(1)}(p a)=\frac{\left[p a h_{n}(p a)\right]^{\prime}}{h_{n}(p a)}$.

In Eqs. (233)-(237), $j_{n}(x)$ and $h_{n}(x)$ are the spherical Bessel and Hankel functions, respectively. From Eqs. (226) and (228), it follows that $\overline{\mathbf{M}}_{p}$ (p) is as in Eq. (209); hence, Assumption A1 of the homogenization theorem is satisfied. Considering the dispersion equation for the longitudinal modes $k_{1}^{2}+n_{0} T_{p \mathrm{~L}}(K)=0$, we see that for small $n_{0}$, we have $n_{0} T_{p \mathrm{~L}}(K) \ll k_{1}^{2}$; hence the dispersion equation for the longitudinal modes has no solutions, and Assumption A2 is also satisfied. If the effective wavenumber is taken as the solution of the equation $K^{2}=k_{1}^{2}+n_{0} T_{p \mathrm{~T}}(K)$ with the smallest positive imaginary part, we conclude that the homogenization theorem is applicable. For this reason, the Foldy approximation for the exciting fields is equivalent to the effective field approximation. Note that if $n_{0}$ is small, the term $n_{0} T_{p \mathrm{~T}}(K)$ is also small, and we can approximate $K \approx k_{1}$. Therefore, the argument $K$ of $T_{p \text { T }}$ can be replaced by $k_{1}$, and we obtain the dispersion relation $K^{2}=k_{1}^{2}+n_{0} T_{p \mathrm{~T}}\left(k_{1}\right)$.

In Ref. [2] it has been shown that the expression for the mass operator derived under the Foldy approximation for the exciting fields is the same as that obtained under the Twersky approximation applied to the Foldy equation for the total field. Both approaches also assume that the positions of the particles are statistically independent. Taking into account that the neglect of the longitudinal waves is allowed in the far-field approximation (when in the far zone, the waves are transverse), we conclude that the effective field approximation is valid under the same assumptions as in Ref. [1].

\section{Effective-field approximation with coherent potential}

In this approach, the counterpart of the transition operator $\overline{\mathbf{T}}_{i}$ for particle $i$ satisfying the Lippmann-Schwinger equation $\overline{\mathbf{T}}_{i}=$ 
$\overline{\mathbf{U}}_{i}+\overline{\mathbf{U}}_{i} \overline{\mathbf{G}}_{0} \overline{\mathbf{T}}_{i}$ is the "effective" transition operator $\overline{\mathbf{T}}_{\mathrm{e} i}$ for particle $i$ satisfying the integral equation $\overline{\mathbf{T}}_{\mathrm{e} i}=\overline{\mathbf{U}}_{i}+\overline{\mathbf{U}}_{i}\langle\overline{\mathbf{G}}\rangle \overline{\mathbf{T}}_{\mathrm{e} i}$. Assuming that $\overline{\mathbf{T}}_{\mathrm{e} i}$ is a translational dyadic, that is, $\overline{\mathbf{T}}_{\mathrm{e} i}\left(\mathbf{r}, \mathbf{r}^{\prime}\right)=\overline{\mathbf{T}}_{\mathrm{e}}\left(\mathbf{r}-\mathbf{R}_{i}, \mathbf{r}^{\prime}-\mathbf{R}_{i}\right)$, where $\overline{\mathbf{T}}_{\mathrm{e}}$ satisfies the Lippmann-Schwinger equation for a particle centered at the origin of the coordinate system $\overline{\mathbf{T}}_{\mathrm{e}}=\overline{\mathbf{U}}_{0}+\overline{\mathbf{U}}_{0}\langle\overline{\mathbf{G}}\rangle \overline{\mathbf{T}}_{\mathrm{e}}$, it is found that in the Fourier space, the mass operator is given by

$\overline{\mathbf{M}}_{p}(\mathbf{p})=n_{0} \overline{\mathbf{T}}_{\mathrm{e} p}(\mathbf{p}, \mathbf{p})$,

where $\overline{\mathbf{T}}_{\mathrm{e} p}\left(\mathbf{p}, \mathbf{p}^{\prime}\right)$ is the Fourier transform of $\overline{\mathbf{T}}_{\mathbf{e}}\left(\mathbf{r}, \mathbf{r}^{\prime}\right)$. The dyadic $\overline{\mathbf{T}}_{\mathrm{e} p}\left(\mathbf{p}, \mathbf{p}^{\prime}\right)$ is computed from the system of equations

$$
\begin{gathered}
\overline{\mathbf{T}}_{\mathrm{e} p}\left(\mathbf{p}, \mathbf{p}^{\prime}\right)=\overline{\mathbf{U}}_{0 p}\left(\mathbf{p}, \mathbf{p}^{\prime}\right)+\frac{1}{(2 \pi)^{3}} \int \overline{\mathbf{U}}_{0 p}\left(\mathbf{p}, \mathbf{p}^{\prime \prime}\right) \\
\cdot \overline{\mathbf{G}}_{p}\left(\mathbf{p}^{\prime \prime}\right) \cdot \overline{\mathbf{T}}_{\mathrm{e} p}\left(\mathbf{p}^{\prime \prime}, \mathbf{p}^{\prime}\right) \mathrm{d}^{3} \mathbf{p}^{\prime \prime},
\end{gathered}
$$

$\overline{\mathbf{G}}_{p}^{-1}(\mathbf{p})=\overline{\mathbf{G}}_{0 p}^{-1}(\mathbf{p})-n_{0} \overline{\mathbf{T}}_{\mathrm{e} p}(\mathbf{p}, \mathbf{p})$.

After solving Eqs. (239)-(240) for $\overline{\mathbf{T}}_{\mathrm{e} p}\left(\mathbf{p}, \mathbf{p}^{\prime}\right)$, the effective wavenumber is determined, in principle, as the solution of the equation (cf. Eq. (208))

$\operatorname{det}\left[\left(K^{2}-k_{1}^{2}\right)(\overline{\mathbf{I}}-\widehat{\mathbf{p}} \otimes \widehat{\mathbf{p}})-k_{1}^{2} \widehat{\mathbf{p}} \otimes \widehat{\mathbf{p}}-n_{0} \overline{\mathbf{T}}_{\mathrm{e} p}(K \widehat{\mathbf{p}}, K \widehat{\mathbf{p}})\right]=0$,

for a specified propagation direction $\widehat{\mathbf{p}}$. Because solving Eqs. (239)(240), and so computing the mass operator by means of Eq. (238), is a difficult task, the following practical approach is used instead. Assume $\langle\overline{\mathbf{G}}\rangle=\overline{\mathbf{G}}_{\mathrm{K}}$ implying $\overline{\mathbf{G}}_{p}=\overline{\mathbf{G}}_{\mathrm{K} p}$, where $\overline{\mathbf{G}}_{\mathrm{K} p}$ is the Fourier transform of the dyadic Green's function $\overline{\mathbf{G}}_{\mathrm{K}}$ corresponding to an infinite homogeneous medium with wavenumber $K$. Next, solve the integral equation

$$
\begin{aligned}
\overline{\mathbf{T}}_{\mathrm{e} p}\left(\mathbf{p}, \mathbf{p}^{\prime} \mid K\right)= & \overline{\mathbf{U}}_{0 p}\left(\mathbf{p}, \mathbf{p}^{\prime}\right)+\frac{1}{(2 \pi)^{3}} \int \overline{\mathbf{U}}_{0 p}\left(\mathbf{p}, \mathbf{p}^{\prime \prime}\right) \\
& \cdot \overline{\mathbf{G}}_{\mathrm{K} p}\left(\mathbf{p}^{\prime \prime}\right) \cdot \overline{\mathbf{T}}_{\mathrm{e} p}\left(\mathbf{p}^{\prime \prime}, \mathbf{p}^{\prime} \mid K\right) \mathrm{d}^{3} \mathbf{p}^{\prime \prime},
\end{aligned}
$$

for any $K$. Thus, $\overline{\mathbf{T}}_{\mathrm{e} p}\left(\mathbf{p}, \mathbf{p}^{\prime} \mid K\right)$ is a parametric solution of parameter $K$. Determine the effective wavenumber as the solution of the equation

$\operatorname{det}\left[\left(K^{2}-k_{1}^{2}\right)(\overline{\mathbf{I}}-\widehat{\mathbf{p}} \otimes \widehat{\mathbf{p}})-k_{1}^{2} \widehat{\mathbf{p}} \otimes \widehat{\mathbf{p}}-n_{0} \overline{\mathbf{T}}_{\mathrm{e} p}(K \widehat{\mathbf{p}}, K \widehat{\mathbf{p}} \mid K)\right]=0$,

and finally, for the solution $K$, compute the mass operator as $\overline{\mathbf{M}}_{p}(\mathbf{p})=n_{0} \overline{\mathbf{T}}_{\mathrm{e} p}(\mathbf{p}, \mathbf{p} \mid K)$. This approach is valid, i.e., the assumption $\langle\overline{\mathbf{G}}\rangle=\overline{\mathbf{G}}_{\mathrm{K}}$ is not contradicted, if the result of the calculation satisfies the assumptions of the homogenization theorem. First of all, $\overline{\mathbf{T}}_{\mathrm{e} p}(\mathbf{p}, \mathbf{p} \mid K)$ should be as in Eq. (228). To show this, we observe that if we rewrite the scattering potential $U_{0}(\mathbf{r})=\left(k_{\mathrm{p}}^{2}-k_{1}^{2}\right) \Theta(\mathbf{r})$ as $U_{0}(\mathbf{r})=\left(\tilde{k}_{\mathrm{p}}^{2}-K^{2}\right) \Theta(\mathbf{r})$, with $\widetilde{k}_{\mathrm{p}}^{2}=k_{\mathrm{p}}^{2}-k_{1}^{2}+K^{2}$, then the transition operator $\overline{\mathbf{T}}_{\mathrm{e}}\left(\mathbf{r}, \mathbf{r}^{\prime} \mid K\right)$ corresponds to a particle of permittivity $\widetilde{\varepsilon}_{\mathrm{p}}=\varepsilon_{\mathrm{p}}-\varepsilon_{1}+\varepsilon_{\text {eff }}$ placed in a medium of permittivity $\varepsilon_{\text {eff }}$, where $\widetilde{k}_{\mathrm{p}}^{2}=\omega^{2} \widetilde{\varepsilon}_{\mathrm{p}} \mu_{0}$ and $K^{2}=\omega^{2} \varepsilon_{\text {eff }} \mu_{0}$. Because in Eqs. (227) and (242), $\overline{\mathbf{G}}_{0 p}(\mathbf{p})$ and $\overline{\mathbf{G}}_{\mathrm{K} p}(\mathbf{p})$ correspond to homogeneous background media with wavenumbers $k_{1}$ and $K$, respectively, we deduce that $\overline{\mathbf{T}}_{\mathrm{e} p}(\mathbf{p}, \mathbf{p} \mid K)$ is as in Eq. (228). More precisley, the expressions for $T_{\mathrm{e} p \mathrm{~L}}(p \mid K)$ and $T_{\mathrm{e} p \mathrm{~T}}(p \mid K)$ are given by EqS. (229)-(232), but in which, $k_{1}$ is replaced by $K, k_{\mathrm{p}}$ by $\widetilde{k}_{\mathrm{p}}$, and $\mathrm{m}$ by $\widetilde{\mathrm{m}}=\widetilde{k}_{\mathrm{p}} / K$. Noting that Assumption A2 of the homogenization theorem is again a consequence of the small value of $n_{0}$, we conclude that if the effective wavenumber is taken as the solution of the equation $K^{2}=k_{1}^{2}+n_{0} T_{\mathrm{e} p \mathrm{~T}}(K \mid K)$ with the smallest positive imaginary part, the assumptions of the homogenization theorem are satisfied, and so that the approximation $\langle\overline{\mathbf{G}}\rangle \approx \overline{\mathbf{G}}_{\mathrm{K}}$ is not contradicted. If we neglect the evanescent waves, the radiative transfer equation for the specific coherency column vector is as in Eq. (184); however, the amplitude matrix corresponds to a spherical particle of permittivity $\widetilde{\varepsilon}_{\mathrm{p}}=\varepsilon_{\mathrm{p}}-\varepsilon_{1}+\varepsilon_{\text {eff }}^{\prime}$ placed in a non-absorbing medium of permittivity $\varepsilon_{\text {eff }}^{\prime}\left(K^{\prime 2}=\omega^{2} \varepsilon_{\text {eff }}^{\prime} \mu_{0}\right)$.

\section{Quasi-crystalline approximation with coherent potential}

The quasi-crystalline approximation or the Lax approximation applies to dense media and relies on the Foldy equations for the total electric field $\mathbf{E}=\mathbf{E}_{0}+\sum_{i} \overline{\mathbf{G}}_{0} \overline{\mathbf{T}}_{i} \mathbf{E}_{\text {exci }}$, and the exciting fields $\mathbf{E}_{\text {exc } i}=\mathbf{E}_{0}+\sum_{j \neq i} \overline{\mathbf{G}}_{0} \overline{\mathbf{T}}_{j} \mathbf{E}_{\text {exc } j}$. In taking the configuration average of these equations, the second-order closure relation or the Lax approximation $\left\langle\mathbf{E}_{\text {exc } j}\right\rangle_{i j}=\left\langle\mathbf{E}_{\text {exc } j}\right\rangle_{j}$ is used. In this approach, the key quantity is the dyadic operator $\overline{\mathbf{C}}_{\mathrm{e} i}$ solving the integral equation

$\overline{\mathbf{C}}_{\mathrm{e} i}=\overline{\mathbf{T}}_{\mathrm{e} i}+n_{0} \int h\left(\mathbf{R}_{j}-\mathbf{R}_{i}\right) \overline{\mathbf{T}}_{\mathrm{e} i}\langle\overline{\mathbf{G}}\rangle \overline{\mathbf{C}}_{\mathrm{e} j} \mathrm{~d}^{3} \mathbf{R}_{j}$,

where $h(\mathbf{r})=g(\mathbf{r})-1$, and $g(\mathbf{r})$ is the correlation function (pairdistribution function) of the discrete scatterers. Assuming that $\overline{\mathbf{C}}_{\mathrm{e} i}$ is a translational dyadic, i.e., $\overline{\mathbf{C}}_{\mathrm{e} i}\left(\mathbf{r}, \mathbf{r}^{\prime}\right)=\overline{\mathbf{C}}_{\mathrm{e}}\left(\mathbf{r}-\mathbf{R}_{i}, \mathbf{r}^{\prime}-\mathbf{R}_{i}\right)$, it is found that in the Fourier space, the mass operator is given by

$\overline{\mathbf{M}}_{p}(\mathbf{p})=n_{0} \overline{\mathbf{C}}_{\mathrm{e} p}(\mathbf{p}, \mathbf{p})$,

where $\overline{\mathbf{C}}_{\mathrm{e} p}\left(\mathbf{p}, \mathbf{p}^{\prime}\right)$ is the Fourier transform of $\overline{\mathbf{C}}_{\mathrm{e}}\left(\mathbf{r}, \mathbf{r}^{\prime}\right)$, and $h_{p}(\mathbf{p})$ is the Fourier transform of $h(\mathbf{r})$. The dyadic $\overline{\mathbf{C}}_{\mathrm{e} p}\left(\mathbf{p}, \mathbf{p}^{\prime}\right)$ satisfies the system of equations

$$
\begin{aligned}
\overline{\mathbf{C}}_{\mathrm{e} p}\left(\mathbf{p}, \mathbf{p}^{\prime}\right)= & \overline{\mathbf{T}}_{\mathrm{e} p}\left(\mathbf{p}, \mathbf{p}^{\prime}\right)+\frac{n_{0}}{(2 \pi)^{3}} \int h_{p}\left(\mathbf{p}^{\prime \prime}-\mathbf{p}^{\prime}\right) \overline{\mathbf{T}}_{\mathrm{e} p}\left(\mathbf{p}, \mathbf{p}^{\prime \prime}\right) \\
& \cdot \overline{\mathbf{G}}_{p}\left(\mathbf{p}^{\prime \prime}\right) \cdot \overline{\mathbf{C}}_{\mathrm{e} p}\left(\mathbf{p}^{\prime \prime}, \mathbf{p}^{\prime}\right) \mathrm{d}^{3} \mathbf{p}^{\prime \prime},
\end{aligned}
$$

$$
\begin{aligned}
\overline{\mathbf{T}}_{\mathrm{e} p}\left(\mathbf{p}, \mathbf{p}^{\prime}\right)= & \overline{\mathbf{U}}_{0 p}\left(\mathbf{p}, \mathbf{p}^{\prime}\right)+\frac{1}{(2 \pi)^{3}} \int \overline{\mathbf{U}}_{0 p}\left(\mathbf{p}, \mathbf{p}^{\prime \prime}\right) \\
& \cdot \overline{\mathbf{G}}_{p}\left(\mathbf{p}^{\prime \prime}\right) \cdot \overline{\mathbf{T}}_{\mathrm{e} p}\left(\mathbf{p}^{\prime \prime}, \mathbf{p}^{\prime}\right) \mathrm{d}^{3} \mathbf{p}^{\prime \prime}
\end{aligned}
$$

$\overline{\mathbf{G}}_{p}^{-1}(\mathbf{p})=\overline{\mathbf{G}}_{0 p}^{-1}(\mathbf{p})-n_{0} \overline{\mathbf{C}}_{\mathrm{e} p}(\mathbf{p}, \mathbf{p})$.

If the preceding approach is used for solving Eqs. (244)-(246), we have to show that $\overline{\mathbf{C}}_{\mathrm{e} p}(\mathbf{p}, \mathbf{p} \mid K)$ is as in Eq. (228). This task is not trivial at all. An alterantive is to compute numerically the dyadic $\overline{\mathbf{C}}_{\mathrm{e} p}\left(\mathbf{p}, \mathbf{p}^{\prime} \mid K\right)$ by using the technique described in Ref. [20], and then to check if $\overline{\mathbf{C}}_{\mathrm{e} p}(\mathbf{p}, \mathbf{p} \mid K)$ possesses this property. In this approach, $\overline{\mathbf{C}}_{\mathrm{e} p}\left(\mathbf{p}, \mathbf{p}^{\prime} \mid K\right)$ and $\overline{\mathbf{T}}_{\mathrm{e} p}\left(\mathbf{p}, \mathbf{p}^{\prime} \mid K\right)$ are expanded in terms of the vector spherical harmonics $\mathbf{v}_{m n}^{\alpha}(\theta, \varphi), \alpha=1,2,3$, that is, for $\mathbf{p}=\left(p, \theta_{p}, \varphi_{p}\right)$ we have

$$
\begin{aligned}
\overline{\mathbf{C}}_{\mathrm{e} p}\left(\mathbf{p}, \mathbf{p}^{\prime} \mid K\right)= & \sum_{m n n^{\prime}} \sum_{\alpha \beta=1,2,3} C_{m n n^{\prime}}^{\alpha \beta}\left(p, p^{\prime} \mid K\right) \\
& \times \mathbf{v}_{m n}^{\alpha}\left(\theta_{p}, \varphi_{p}\right) \otimes \mathbf{v}_{-m n^{\prime}}^{\beta}\left(\theta_{p^{\prime}}, \varphi_{p^{\prime}}\right)
\end{aligned}
$$

and

$$
\begin{aligned}
\overline{\mathbf{T}}_{\mathrm{e} p}\left(\mathbf{p}, \mathbf{p}^{\prime} \mid K\right)= & \sum_{m n n^{\prime}} \sum_{\alpha \beta=1,2,3} T_{m n n^{\prime}}^{\alpha \beta}\left(p, p^{\prime} \mid K\right) \\
& \times \mathbf{v}_{m n}^{\alpha}\left(\theta_{p}, \varphi_{p}\right) \otimes \mathbf{v}_{-m n^{\prime}}^{\beta}\left(\theta_{p^{\prime}}, \varphi_{p^{\prime}}\right),
\end{aligned}
$$

respectively, and then the integral equation (244) is transformed into an algebraic system of equations for the expansion functions $C_{m n n^{\prime}}^{\alpha \beta}\left(p, p^{\prime} \mid K\right)$. The solution of this system of equations for all frequencies is extremely laborious. However, in the lowfrequency limit, an analytic solution can be obtained; it is given 
by $\overline{\mathbf{C}}_{\mathrm{e} p}\left(\mathbf{p}, \mathbf{p}^{\prime} \mid K\right)=C_{\mathrm{e}}(K) \overline{\mathbf{I}}$ for any $\mathbf{p}$ and $\mathbf{p}^{\prime}$, where

$C_{\mathrm{e}}(K)=\frac{z V_{0}}{1+\frac{z(1-f)}{3 K^{2}}}\left\{1+\frac{2 \mathrm{j}}{9} \frac{z K a^{3}}{1+\frac{z(1-f)}{3 K^{2}}}\left[1+4 \pi n_{0} \int_{0}^{\infty} h(r) r^{2} \mathrm{~d} r\right]\right\}$,

$f=n_{0} V_{0}$ is the particle volume concentration, and $z=k_{1}^{2}\left(\mathrm{~m}^{2}-1\right)$. Thus, in the low-frequency limit $\overline{\mathbf{C}}_{\mathrm{e} p}(\mathbf{p}, \mathbf{p} \mid K)$ is as in Eq. (228), and therefore, the effective field approximation is valid in this limit. In fact, in the limit $p \rightarrow 0, \overline{\mathbf{C}}_{\mathrm{e} p}(\mathbf{p}, \mathbf{p} \mid K)$ playing the role of the transition operator does not depend on $\mathbf{p}$ at all (see the above formula). The reason is that, relative to the wavelength, the sphere becomes a point-like scatterer, and a point has no length scale and no geometric structure to speak of.

For dense media and all frequencies, it is not only Assumption A1 but also Assumption A2 which appears to be problematic. The neglect of the longitudinal waves is equivalent to the far-field approximation, which, in turn, is typical of sparse media. Therefore, we believe that the use of the homogenization theorem in the case of dense media leads only to an approximate model for describing radiative transfer (essentially, the model borrows some features which are characteristic of sparse media). Such a model [14] uses the correlated ladder approximation for the Bethe-Salpeter equation in which the tetradic scattering intensity operator is given by

$$
\begin{aligned}
\overline{\overline{\mathbf{I}}}\left(\mathbf{r}, \mathbf{r}_{1}, \mathbf{r}^{\prime}, \mathbf{r}_{1}^{\prime}\right)= & n_{0} \int_{D_{1}} \overline{\mathbf{C}}_{\mathrm{e} i}\left(\mathbf{r}, \mathbf{r}_{1}\right) \otimes \overline{\mathbf{C}}_{\mathrm{e} i}^{\star}\left(\mathbf{r}^{\prime}, \mathbf{r}_{1}^{\prime}\right) \mathrm{d}^{3} \mathbf{R}_{i} \\
& +n_{0}^{2} \int_{D_{1}} h\left(\mathbf{R}_{j}-\mathbf{R}_{i}\right) \overline{\mathbf{C}}_{\mathrm{e} i}\left(\mathbf{r}, \mathbf{r}_{1}\right) \otimes \overline{\mathbf{C}}_{\mathrm{e} j}^{\star}\left(\mathbf{r}^{\prime}, \mathbf{r}_{1}^{\prime}\right) \mathrm{d}^{3} \mathbf{R}_{j} \mathrm{~d}^{3} \mathbf{R}_{i} .
\end{aligned}
$$

The Wigner-Fourier transform of the intensity operator at $\mathbf{P}_{\perp}=0$ is (compare to Eq. (118))

$$
\begin{aligned}
\overline{\overline{\mathbf{I}}}_{\mathrm{WF}}\left(Z, \mathbf{p}, Z_{1}, \mathbf{p}_{1}, 0\right) & =n_{0} q_{p}\left(\mathbf{p}_{1}-\mathbf{p}\right) \delta\left(Z-Z_{1}\right) \overline{\mathbf{C}}_{\mathrm{e} p}\left(\mathbf{p}^{\operatorname{sgn}\left(p_{\mathrm{z}}\right)}, \mathbf{p}_{1}^{\operatorname{sgn}\left(p_{1 \mathrm{z}}\right)}\right) \\
& \otimes \overline{\mathbf{C}}_{\mathrm{e} p}^{\star}\left(\mathbf{p}^{\operatorname{sgn}\left(p_{\mathrm{z}}\right)}, \mathbf{p}_{1}^{\operatorname{sgn}\left(p_{1 \mathrm{z}}\right)}\right),
\end{aligned}
$$

where $q_{p}(\mathbf{p})=n_{0} h_{p}(\mathbf{p})+1$. By employing essentially the same arguments as in the derivation of the integral equation (151), we find that the integral form of the radiative transfer equation for the transverse components $\overline{\mathscr{C}}^{a a}\left(Z, \mathbf{p}_{\perp}\right)$ of the Wigner transform $\overline{\mathscr{C}}_{\mathrm{W}}(Z, \mathbf{p})$, is

$$
\begin{aligned}
& \overline{\mathscr{C}}^{a a}\left(Z, \mathbf{p}_{\perp}\right) \\
& =\frac{n_{0}}{16 \pi^{2}} \mathrm{e}^{-2 a K_{\mathrm{z}}^{\prime \prime}\left(\mathbf{p}_{\perp}\right) Z} \sum_{b, c= \pm} \int_{0}^{H}\left\{\int \frac{1}{\left|K_{\mathrm{z}}\left(\mathbf{p}_{1 \perp}\right)\right|^{2}} \mathrm{e}^{2 b K_{\mathrm{z}}^{\prime \prime}\left(\mathbf{p}_{1 \perp}\right) Z_{1}}\right. \\
& \quad \times q_{p}\left(\mathbf{p}_{2 \perp}-\mathbf{p}_{1 \perp}, c K_{\mathrm{z}}^{\prime}\left(\mathbf{p}_{2 \perp}\right)-b K_{\mathrm{z}}^{\prime}\left(\mathbf{p}_{1 \perp}\right)\right) \\
& \quad \times\left[\overline{\mathcal{\mathcal { S }}}^{1 a 1 b}\left(\mathbf{p}_{\perp}, \mathbf{p}_{1 \perp}, 0\right)+\delta_{a b} \delta_{a, \operatorname{sgn}\left(Z-Z_{1}\right)} \delta\left(\mathbf{p}_{\perp}-\mathbf{p}_{1 \perp}\right) \overline{\overline{\mathbf{I}}}_{\perp}^{a b}\left(\mathbf{p}_{\perp}\right)\right] \\
& \left.\quad \cdot \overline{\overline{\mathbf{C}}}_{\mathrm{e} p \mathrm{~T}}^{b c}\left(\mathbf{p}_{1 \perp}, \mathbf{p}_{2 \perp}\right) \cdot\left[\overline{\mathbf{C}}_{\mathrm{c}}^{c c}\left(Z_{1}, \mathbf{p}_{2 \perp}\right)+\overline{\mathscr{C}}^{c c}\left(Z_{1}, \mathbf{p}_{2 \perp}\right)\right] \mathrm{d}^{2} \mathbf{p}_{1 \perp} \mathrm{d}^{2} \mathbf{p}_{2 \perp}\right\} \mathrm{d} Z_{1},
\end{aligned}
$$

where (compare to Eq. (152))

$\overline{\overline{\mathbf{C}}}_{\mathrm{e} p \mathrm{~T}}^{b c}\left(\mathbf{p}_{1 \perp}, \mathbf{p}_{2 \perp}\right)=\overline{\mathbf{C}}_{\mathrm{e} p \mathrm{~T}}\left(\mathbf{p}_{1}^{b}, \mathbf{p}_{2}^{c}\right) \otimes \overline{\mathbf{C}}_{\mathrm{e} p \mathrm{~T}}^{\star}\left(\mathbf{p}_{1}^{b}, \mathbf{p}_{2}^{c}\right)$,

and we have set $q_{p}\left(\mathbf{p}_{2}-\mathbf{p}_{1}\right)=q_{p}\left(\mathbf{p}_{2 \perp}-\mathbf{p}_{1 \perp}, p_{2 z}-p_{1 z}\right)$. Taking the derivative of Eq. (250), we get (compare to Eq. (159))

$$
\begin{aligned}
\frac{\partial}{\partial Z} \overline{\mathscr{C}}^{a a}\left(Z, \mathbf{p}_{\perp}\right) & =-2 a K_{\mathrm{z}}^{\prime \prime}\left(\mathbf{p}_{\perp}\right) \overline{\mathscr{C}}^{a a}\left(Z, \mathbf{p}_{\perp}\right)+\frac{a}{16 \pi^{2}} \frac{n_{0}}{\left|K_{\mathrm{z}}\left(\mathbf{p}_{\perp}\right)\right|^{2}} \\
& \times \sum_{b= \pm} \int q_{p}\left(\mathbf{p}_{1 \perp}-\mathbf{p}_{\perp}, b K_{\mathrm{z}}^{\prime}\left(\mathbf{p}_{1 \perp}\right)-a K_{\mathrm{z}}^{\prime}\left(\mathbf{p}_{\perp}\right)\right) \\
& \times \overline{\overline{\mathbf{C}}}_{\mathrm{e} p \mathrm{~T}}^{a b}\left(\mathbf{p}_{\perp}, \mathbf{p}_{1 \perp}\right) \cdot\left[\overline{\mathbf{C}}_{\mathrm{c}}^{b b}\left(Z, \mathbf{p}_{1 \perp}\right)+\overline{\mathscr{C}}^{b b}\left(Z, \mathbf{p}_{1 \perp}\right)\right] \mathrm{d}^{2} \mathbf{p}_{1 \perp} .
\end{aligned}
$$

For sparse media, we have $q_{p}\left(\mathbf{p}_{2}-\mathbf{p}_{1}\right)=1$ and $\overline{\mathbf{C}}_{\mathrm{e} p \mathrm{~T}}\left(\mathbf{p}_{1}^{b}, \mathbf{p}_{2}^{c}\right)=$ $\overline{\mathbf{T}}_{p \mathrm{~T}}\left(\mathbf{p}_{1}^{b}, \mathbf{p}_{2}^{c}\right)$; consequently, Eqs. (250) and (252) reduce to Eqs. (151) and (159), respectively. On the other hand, in the low-frequency limit and because the function $h(\mathbf{r})=g(\mathbf{r})-1$ is nonzero over a distance range of several particle diameters $(h(\mathbf{r})$ is sharply picked on the mean-free path scale $l_{\mathrm{mp}}$ ), the Wigner transform of the intensity operator has a particular form, which in turn, implies that the integral equation for $\overline{\mathscr{C}}^{a a}\left(Z, \mathbf{p}_{\perp}\right)$ is as in Eq. (250) with $q_{p}(0)$ in place of $q_{p}\left(\mathbf{p}_{2}-\mathbf{p}_{1}\right)$, and $\overline{\overline{\mathbf{C}}}_{\mathrm{e} p \mathrm{~T}}^{b c}\left(\mathbf{p}_{1 \perp}, \mathbf{p}_{2 \perp}\right)=$ $\left|C_{\mathrm{e}}\left(K^{\prime}\right)\right|^{2} \overline{\mathbf{I}}_{\perp}\left(\mathbf{p}_{1 \perp}\right) \otimes \overline{\mathbf{I}}_{\perp}\left(\mathbf{p}_{2 \perp}\right)$. Moreover, if the evanescent waves are neglected, the radiative transfer equation for the specific coherency column vector is as in Eq. (184) with the coherency phase matrix

$\left[\mathrm{Z}_{\mathrm{J}}\left(\widehat{\mathbf{p}}, \widehat{\mathbf{p}}_{1}\right)\right]_{(\eta, \mu)\left(\eta^{\prime}, \mu^{\prime}\right)}=\frac{q_{p}(0)\left|C_{\mathrm{e}}\left(K^{\prime}\right)\right|^{2}}{16 \pi^{2}} f_{\eta \eta^{\prime} \mu \mu^{\prime}}\left(\widehat{\mathbf{p}}, \widehat{\mathbf{p}}_{1}\right)$,

$f_{\eta \eta^{\prime} \mu \mu^{\prime}}\left(\widehat{\mathbf{p}}, \widehat{\mathbf{p}}_{1}\right)=\left[\widehat{\boldsymbol{\eta}}(\widehat{\mathbf{p}}) \cdot \widehat{\boldsymbol{\eta}}^{\prime}\left(\widehat{\mathbf{p}}_{1}\right)\right]\left[\widehat{\boldsymbol{\mu}}(\widehat{\mathbf{p}}) \cdot \widehat{\boldsymbol{\mu}}^{\prime}\left(\widehat{\mathbf{p}}_{1}\right)\right]$,

for $\eta, \eta^{\prime}, \mu, \mu^{\prime}=\theta, \varphi$.

\section{References}

[1] Doicu A, Mishchenko MI. Overview of methods for deriving the radiative transfer theory from the Maxwell equations. I: Approach based on the far-field Foldy equations. J Quant Spectrosc Radiat Transfer 2018;220:123-39.

[2] Doicu A, Mishchenko MI. An overview of methods for deriving the radiative transfer theory from the Maxwell equations. II: Approach based on the Dyson and Bethe-Salpeter equations. J Quant Spectrosc Radiat Transfer, in press 2018.

[3] Mishchenko MI, Travis LD, Lacis AA. Multiple scattering of light by particles: radiative transfer and coherent backscattering. Cambridge, UK: Cambridge University Press; 2006.

[4] Mishchenko MI. Electromagnetic scattering by particles and particle groups: an introduction. Cambridge, UK: Cambridge University Press; 2014. < https: //www.giss.nasa.gov/staff/mmishchenko/publications/Book_4.pdf>.

[5] Walther A. Radiometry and coherence. J Opt Soc Am 1968:58:1256-9.

[6] Marchand EW, Wolf E. Radiometry with sources in any state of coherence. J Opt Soc Am 1974;64:1219-26.

[7] Apresyan LA, Kravtsov YA. Photometry and coherence: wave aspects of the theory of radiation transport. Sov Phys Usp 1984;27:301-13.

[8] Apresyan LA, Kravtsov YA. Radiation transfer: statistical and wave aspects. Amsterdam: Gordon and Breach; 1996.

[9] Yoshimori K. Radiometry and coherence in a nonstationary optical field. J Opt Soc Am 1998;15:2730-4

[10] Tsang L, Ishimaru A. Radiative wave equations for vector electromagnetic propagation in dense nontenuous media. J Electromagn Waves Appl 1987;1:59-72.

[11] Tsang L, Kong JA. Scattering of electromagnetic waves. Advanced topics. New York: Wiley; 2001

[12] Jin YQ. Some results from the radiative wave equation for a slab of random, densely-distributed scatterers. J Quant Spectrosc Radiat Transfer 1988;39:83-98.

[13] Soubret A, Berginc G. Electromagnetic wave scattering from a random layer with rough interfaces I: coherent field. ArXiv:physics/0312133 [physics.ao-ph].

[14] Soubret A, Berginc G. Electromagnetic wave scattering from a random layer with rough interfaces II: diffusive intensity. ArXiv:physics/0312136 [physics.aoph].

15] Voronovich AG. Wave scattering from rough surfaces. Berlin: Springer; 1999.

[16] Mudaliar S. Remote sensing of layered random media using the radiative transfer theory. Radio Sci 2013;48:535-46.

[17] Barabanenkov YN, Kravtsov YA, Ozrin VD, Saichev AI. Enhanced backscattering in optics. Prog Opt 1991:29:65-197.

[18] Soubret A, Berginc G. Effective dielectric constant for a random medium. ArXiv:physics/0312117 [physics.optics].

[19] Pellegrini YP, Thibaudeau P, Stout B. The off-shell electromagnetic t-matrix: momentum-dependent scattering from spherical inclusions with both dielectric and magnetic contrast. Waves Random Complex Media 2011;21:313-35.

[20] Tsang L, Kong JA. Multiple scattering of electromagnetic waves by random distribution of discrete scatterers with coherent potential and quantum mechanical formalism. J Appl Phys 1980;51:3465-85. 